\title{
A HERSCHEL* AND APEX CENSUS OF THE REDDEST SOURCES IN ORION: SEARCHING FOR THE YOUNGEST PROTOSTARS ${ }^{\dagger}$
}

\author{
Amelia M. Stutz ${ }^{1}$, John J. Tobin ${ }^{2,13}$, Thomas Stanke ${ }^{3}$, S. Thomas Megeath ${ }^{4}$, William J. Fischer ${ }^{4}$, Thomas Robitaille ${ }^{1}$, \\ Thomas Henning ${ }^{1}$, Babar Ali $^{5}$, James di Francesco ${ }^{6,7}$, Elise Furlan ${ }^{5,8}$, Lee Hartmann ${ }^{9}$, Mayra Osorio $^{10}$, \\ Thomas L. Wilson ${ }^{11}$, Lori Allen ${ }^{8}$, Oliver Krause ${ }^{1}$, and P. Manoj ${ }^{12}$ \\ ${ }^{1}$ Max Planck Institute for Astronomy, Königstuhl 17, D-69117 Heidelberg, Germany; stutz@mpia.de \\ 2 National Radio Astronomy Observatory, Charlottesville, VA 22903, USA \\ ${ }^{3}$ ESO, Karl-Schwarzschild-Strasse 2, D-85748 Garching bei München, Germany \\ ${ }^{4}$ Department of Physics and Astronomy, University of Toledo, 2801 W. Bancroft Street, Toledo, OH 43606, USA \\ 5 NHSC/IPAC/Caltech, 770 S. Wilson Avenue, Pasadena, CA 91125, USA \\ ${ }^{6}$ National Research Council of Canada, Herzberg Institute of Astrophysics, 5071 West Saanich Road, Victoria, BC V9E 2E7, Canada \\ ${ }^{7}$ Department of Physics and Astronomy, University of Victoria, P.O. Box 3055, STN CSC, Victoria, BC V8W 3P6, Canada \\ ${ }^{8}$ National Optical Astronomy Observatory, 950 North Cherry Avenue, Tucson, AZ 85719, USA \\ ${ }^{9}$ Department of Astronomy, University of Michigan, 830 Dennison Building, 500 Church Street, Ann Arbor, MI 48109, USA \\ ${ }^{10}$ Instituto de Astrofísica de Andalucía, CSIC, Camino Bajo de Huétor 50, E-18008 Granada, Spain \\ ${ }^{11}$ Naval Research Laboratory, 4555 Overlook Avenue SW, Washington, DC 20375, USA \\ ${ }^{12}$ Department of Physics and Astronomy, 500 Wilson Boulevard, University of Rochester, Rochester, NY 14627, USA \\ Received 2012 December 3; accepted 2013 January 26; published 2013 March 22
}

\begin{abstract}
We perform a census of the reddest, and potentially youngest, protostars in the Orion molecular clouds using data obtained with the PACS instrument on board the Herschel Space Observatory and the LABOCA and SABOCA instruments on APEX as part of the Herschel Orion Protostar Survey (HOPS). A total of 55 new protostar candidates are detected at $70 \mu \mathrm{m}$ and $160 \mu \mathrm{m}$ that are either too faint $\left(m_{24}>7 \mathrm{mag}\right)$ to be reliably classified as protostars or undetected in the Spitzer/MIPS $24 \mu \mathrm{m}$ band. We find that the 11 reddest protostar candidates with log $\lambda F_{\lambda} 70 / \lambda F_{\lambda} 24>1.65$ are free of contamination and can thus be reliably explained as protostars. The remaining 44 sources have less extreme $70 / 24$ colors, fainter $70 \mu \mathrm{m}$ fluxes, and higher levels of contamination. Taking the previously known sample of Spitzer protostars and the new sample together, we find 18 sources that have log $\lambda F_{\lambda} 70 / \lambda F_{\lambda} 24>1.65$; we name these sources "PACS Bright Red sources," or PBRs. Our analysis reveals that the PBR sample is composed of Class 0 like sources characterized by very red spectral energy distributions (SEDs; $\left.T_{\text {bol }}<45 \mathrm{~K}\right)$ and large values of sub-millimeter fluxes $\left(L_{\mathrm{smm}} / L_{\mathrm{bol}}>0.6 \%\right)$. Modified blackbody fits to the SEDs provide lower limits to the envelope masses of $0.2-2 M_{\odot}$ and luminosities of $0.7-10 L_{\odot}$. Based on these properties, and a comparison of the SEDs with radiative transfer models of protostars, we conclude that the PBRs are most likely extreme Class 0 objects distinguished by higher than typical envelope densities and hence, high mass infall rates.
\end{abstract}

Key words: ISM: clouds - stars: formation - stars: low-mass - stars: protostars

Online-only material: color figures

\section{INTRODUCTION}

The onset of the star formation process is broadly characterized by a dense collapsing cloud envelope surrounding the nascent protostar. The dense cloud or protostellar envelope is opaque to radiation shortward of about $\sim 10 \mu \mathrm{m}$ and most of the radiation from these sources is reprocessed and emitted in the far-infrared (FIR). Furthermore, bipolar outflows from the protostar and disk carve out envelope cavities that enable a fraction of the protostellar luminosity to escape in the form of scattered light emission, predominantly at wavelengths shortward of $\sim 10 \mu \mathrm{m}$.

The earliest phase of protostellar evolution, the Class 0 phase (André et al. 1993), is thought to be short compared to the

\footnotetext{
* Herschel is an ESA space observatory with science instruments provided by European-led Principal Investigator consortia and with important participation from NASA.

$\dagger$ Based (in part) on observations collected at the European Organisation for Astronomical Research in the Southern Hemisphere, Chile, proposals E-284.C-0515, E-086.C-0848, E-088.C-0994, and E-090.C-0894.

${ }^{13}$ Hubble Fellow.
}

Class I phase (Lada 1987), with combined Class 0 and Class I lifetimes of $\sim 0.5 \mathrm{Myr}$ (Evans et al. 2009); these estimates assume a constant star formation rate and a typical age for Class II objects (pre-main-sequence stars with disks) of $2 \mathrm{Myr}$. At the onset of collapse and immediately before the Class 0 phase, protostars may go through a brief first hydrostatic core (FHSC) phase where the forming protostellar object becomes opaque to its own radiation for the first time (Larson 1969). The FHSC is expected to be very low luminosity and deeply embedded. A population of very low luminosity protostars (VeLLOs) was also recently identified by Spitzer (e.g., Dunham et al. 2008; Bourke et al. 2006), defined to have model-estimated internal source luminosities of less than $0.1 L_{\odot}$. VeLLOs, however, appear more evolved than FHSCs with features consistent with Class 0 and I protostars. Furthermore, while several FHSC candidates have been identified recently (e.g., see Enoch et al. 2010; Chen et al. 2010; Pineda et al. 2011; Pezzuto et al. 2012), it has proven observationally difficult to distinguish such sources from the young Class 0 protostellar phase. It is therefore currently difficult to identify the very earliest phases of the formation of a protostar. 
Before the launch of Spitzer and the advent of extremely sensitive mid-infrared surveys, conventional wisdom held that a Class 0 protostar should not be detectable at wavelengths shortward of $10 \mu \mathrm{m}$ due to the envelope opacity (Williams \& Cieza 2011). Outflows, however, can carve out cavities in the protostellar envelopes at a very early age and are expected to widen with evolution (Arce \& Sargent 2006). Indeed, recent simulations have shown that even the extremely young FHSC sources may be capable of driving outflows (Commerçon et al. 2012; Price et al. 2012). Regardless of evolutionary state, the outflow cavities enable near- to mid-infrared light from the protostar and disk to escape and scatter off dust grains in the cavity or on the cavity walls. This phenomenon has been well known for Class I sources (Kenyon et al. 1993; Padgett et al. 1999), but Class 0 protostars were only well detected in the midinfrared with Spitzer (Noriega-Crespo et al. 2004; Jørgensen et al. 2007; Stutz et al. 2008). The scattered light from Class 0 protostars is often brightest at wavelengths $\sim 3.6 \mu \mathrm{m}$ or $4.5 \mu \mathrm{m}$ due to the dense envelope obscuration at shorter wavelengths (e.g., Whitney et al. 2003b; Tobin et al. 2007).

The combination of results from recent space- and groundbased surveys have resulted in well-sampled spectral energy distributions (SEDs) from the near-infrared to the (sub-)millimeter for large samples of protostellar objects (e.g., Hatchell et al. 2007; Enoch et al. 2009; Launhardt et al. 2010, 2013; Fischer et al. 2010). These SEDs are dominated by scattered light between $\sim 1 \mu \mathrm{m}$ and $10 \mu \mathrm{m}$, optically thick thermal dust emission from $\sim 10$ to $\sim 160 \mu \mathrm{m}$, and optically thin dust emission at wavelengths longward of $\sim 160 \mu \mathrm{m}$. Radiative transfer models of protostellar collapse have become increasingly important to interpret these data since these can account for the varying temperature and density profiles in the envelopes surrounding the protostar (e.g., Whitney et al. 2003a, 2003b).

The large number of free model parameters-such as the combination of outflow cavities, rotationally flattened envelopes, (Ulrich 1976; Cassen \& Moosman 1981; Terebey et al. 1984), and varying viewing angles-can however make the best-fit SED model parameters highly degenerate (e.g., Whitney et al. $2003 b$ ). For example, sources viewed at nearly edge-on orientations can be substantially more obscured than sources at the same evolutionary state viewed from a less extreme vantage point. Thus, standard diagnostics such as bolometric temperature or mid-infrared spectral index can yield vastly different results depending on the source inclination (e.g., Dunham et al. 2010). Radiative transfer models can help break some of these degeneracies but ambiguities can remain as to whether a source has a very dense envelope or if it is simply viewed edge-on.

While much has been learned about the Class 0 phase from observations and modeling, there are relatively few Class 0 objects present in nearby star-forming clouds and globules (Evans et al. 2009) compared to the numbers of Class I and Class II sources. One of the principal goals of recent star formation surveys has been to understand the evolution of protostellar sources. Young \& Evans (2005) generated models for the smooth luminosity evolution of protostellar objects that will become $0.3 M_{\odot}, 1 M_{\odot}$, and $3 M_{\odot}$ stars. These models, however, overpredict the luminosities of most protostars located in nearby star-forming regions, a fact that is taken as evidence for episodic accretion (Kenyon \& Hartmann 1990; Evans et al. 2009; Dunham et al. 2010). However, Offner \& McKee (2011) show that the observed luminosity functions of protostars can be explained through a dependence of the mass accretion rate on the instantaneous and final mass of the protostar. The low resolutions and sensitivities of previous FIR instrumentation have made the detection of protostars in more distant and richer star-forming regions difficult and subject to substantial confusion. Thus, studies of protostellar evolution have been limited to combining all known Class 0 protostars from the nearby regions into a single analysis (e.g., Myers et al. 1998; Evans et al. 2009) to achieve a more robust sample size.

The advent of the Herschel Space Observatory (Pilbratt et al. 2010) has tremendously improved resolution and sensitivity to FIR radiation, where protostars emit the bulk of their energy. These improvements enable the study of protostellar populations to be extended to more distant, richer regions of star formation that have more statistically significant samples of protostars in the Class 0 and I phases (e.g., Ragan et al. 2012). The Herschel Orion Protostar Survey (HOPS) is a Herschel Open Time Key Programme (OTKP; e.g., Stanke et al. 2010; Fischer et al. 2010; Ali et al. 2010; Manoj et al. 2013) targeting 300 of the Spitzer-identified Orion protostars with PACS (Poglitsch et al. 2010) $70 \mu \mathrm{m}$ and $160 \mu \mathrm{m}$ photometry and PACS spectroscopy (53-200 $\mu \mathrm{m}$; Manoj et al. 2013) for a subset of 30 protostars. Orion is the richest star-forming region within $500 \mathrm{pc}$ of the Sun (Megeath et al. 2012), at a distance of $\sim 420$ pc (average value; Menten et al. 2007; Hirota et al. 2007; Sandstrom et al. 2007). The large sample of protostars in Orion enables studies of protostellar evolution to be carried out for a single star-forming complex where all protostars lie at nearly the same distance with a statistically significant sample, comparable to or larger than all the nearby regions combined. The large sample may also enable short timescale phenomena (e.g., Fischer et al. 2012) to be detected, such as brief periods of high envelope infall rate in the earliest phases of star formation. Even with the increased numbers of protostars in Orion, however, it is unclear if we would expect to detect FHSCs given the faintness of these sources and short lifetimes of less than $10 \mathrm{kyr}$ (Commerçon et al. 2012).

The PACS imaging of the HOPS program has the potential to identify protostars that were not detected by Spitzer due to a combination of opacity of the envelope and/or confusion with nearby sources. Indeed, the PACS $70 \mu \mathrm{m}$ band of Herschel is ideal for detecting such protostars, with the highest angular resolution, limiting the blending of sources. Also, the lower opacity relative to MIPS $24 \mu \mathrm{m}$ allows the reprocessed warm inner envelope radiation to escape. Finally, and most importantly, a $70 \mu \mathrm{m}$ point source is strong evidence for an embedded protostar because external heating cannot raise temperatures high enough to emit at this wavelength. Thus, some cores in sub-millimeter surveys that were previously identified as starless may in reality be protostellar.

Using Herschel, we have serendipitously identified a sample of $70 \mu \mathrm{m}$ point sources that were not identified at shorter wavelengths in the previous Spitzer protostar sample (Megeath et al. 2012). Furthermore, we have identified a subset of these that have the reddest $70 \mu \mathrm{m}$ to $24 \mu \mathrm{m}$ colors of all protostars in the combined Orion sample. These sources may have the densest envelopes and are possibly the youngest detected Orion protostars and we name them "PACS Bright Red sources" or PBRs.

We will describe the methodology of identifying these sources and classify them as either being protostellar, extragalactic contamination, or spurious detections coincident with extended emission. We will discuss the observations and data reduction in Section 2, the source finding and classification methods in Section 3, the observed properties of the new sources in 
Section 4, the PBRs in Section 5, the comparison of the cold PBRs to models in Section 6, some relevant model degeneracies in Section 7, and, finally, our results in Section 8. Throughout this work, all positions are given in the J2000 system.

\section{OBSERVATIONS, DATA REDUCTION, AND PHOTOMETRY}

In this work, we present Herschel scan-map observations of a subset of the HOPS fields containing candidate protostars. In addition, we present a subset of our APEX LABOCA and SABOCA observations of these fields. A summary of the HOPS Herschel PACS survey observations is presented in Tables 1 and 2. Here we discuss the observations, data processing, and photometry extraction.

\subsection{Herschel PACS}

The PACS data were acquired simultaneously at $70 \mu \mathrm{m}$ and $160 \mu \mathrm{m}$ over $5^{\prime} \times 5^{\prime}$ or $8^{\prime} \times 8^{\prime}$ field sizes. The field sizes and centers were chosen to maximize observing efficiency by allowing each field to include as many of the target Spitzer-selected protostars (Megeath et al. 2012) as possible while minimizing redundant coverage. The observations were acquired at medium scan speed $\left(20^{\prime \prime} \mathrm{s}^{-1}\right)$, and are composed of two orthogonal scans with homogeneous coverage.

The PACS data were reduced using the Herschel Interactive Processing Environment (HIPE) version 8.0 build 248 and version 9.0 build 215 . We used a custom-built pipeline to process data from their raw form (the so-called Level 0 data) to fully calibrated time lines (Level 1) just prior to the map-making step. Our pipeline uses the same processing steps as described by Poglitsch et al. (2010) but also include the following additions and modifications. First, we used a spatial redundancy-based algorithm to identify and mask cosmic-ray hits. Second, we mitigated instrument cross-talk artifacts by masking (flagging as unusable) detector array columns affected by cross-talk noise. This technique is effective but at the expense of loss of signal from the affected detector array columns. Third, we used the "FM6" version of the instrument responsivity, which has a direct bearing on the absolute calibration of the final mosaics.

The Level 1 data were processed with "Scanamorphos" (Roussel 2012) version 14.0. The final maps were produced using the galactic option and included the turnaround (non-zero acceleration) data. The final map pixel scales used in this work are $1^{\prime \prime} .0$ pixel $^{-1}$ at $70 \mu \mathrm{m}$ and $2^{\prime \prime} .0$ pixel $^{-1}$ at $160 \mu \mathrm{m}$.

The photometry was performed in the following fashion. We first derived customized aperture corrections to the $70 \mu \mathrm{m}$ and $160 \mu \mathrm{m}$ data using the Herschel Science Center (HSC) provided observations of Vesta (to be discussed in more detail in W. J. Fischer et al., in preparation). At $70 \mu \mathrm{m}$, we used radii of sizes 9.'6, 9.'6, and 19'.2, for the aperture and sky annuli, respectively. For these parameters we derived an aperture correction of 0.7331 , where the measured flux in the aperture is divided by this correction to obtain a total point-source flux. At $160 \mu \mathrm{m}$, we used aperture radii of $12^{\prime \prime} .8,12^{\prime \prime} .8$, and $25^{\prime \prime} .6$, for the aperture and sky annuli, respectively. Similarly, we derived an aperture correction of 0.6602 . The encircled energy fractions provided by the photApertureCorrectionPointSource task in HIPE do not account for the effect of applying an inner sky annulus that is close to the size of the source aperture and small compare to the point-spread function (PSF). Our corrections therefore account for $3 \%-4 \%$ of the source flux that is removed. Furthermore, our adopted aperture sizes are smaller than the
PACS instrument team recommendation but were chosen to minimize contribution from nebulosity (extended, non-pointlike emission) often surrounding the protostars in Orion. Given the complex structure in the images and at times crowded fields, our aperture photometry may suffer from blending and contamination. The photometric errors include a $10 \%$ systematic error floor added in quadrature to the standard photometric uncertainties. These errors represent systematic uncertainties in our photometry and aperture correction, as well as the overall calibration uncertainty of PACS. We note that the reported HSC point-source calibration uncertainties for PACS are $\sim 3 \%$ at $70 \mu \mathrm{m}$ and $\sim 5 \%$ at $160 \mu \mathrm{m}$, and were derived from isolated photometric standards. Therefore, our final uncertainties are conservative.

We include the $100 \mu \mathrm{m}$ Gould Belt Survey (GBS; e.g., André et al. 2010; Könyves et al. 2010; Men'shchikov et al. 2010, see also N. Schneider et al., in preparation, for Orion B, and A. Roy et al., in preparation, and D. Polychroni et al., in preparation, for Orion A) data of Orion in this work for the PBRs analysis. Given the sparsely covered SEDs of our sources, these data provide important information regarding the shape of the thermal SED of cold envelope sources. These data were acquired using medium scan speed $\left(20^{\prime \prime} \mathrm{s}^{-1}\right)$ and cover an area much larger than the HOPS fields. These data were processed in a similar way to the HOPS processing described above. Following the above $70 \mu \mathrm{m}$ and $160 \mu \mathrm{m}$ analysis, we used aperture radii of sizes 9.'6, 9.'6, and $19^{\prime \prime} .2$ for the aperture and sky annuli, respectively. For these parameters, we derived an aperture correction of 0.6944 . As with the $70 \mu \mathrm{m}$ and $160 \mu \mathrm{m}$ data, we also assume a conservative $10 \%$ systematic error floor.

\subsection{APEX SABOCA and $L A B O C A$}

We obtained sub-millimeter (smm) continuum maps using the LABOCA and SABOCA bolometer arrays on the APEX telescope. LABOCA (Siringo et al. 2009) is a $\sim 250$ bolometer array operating at $870 \mu \mathrm{m}$, with a spatial resolution of $\sim 19^{\prime \prime}$ at FWHM. We used a combination of spiral and straight onthe-fly scans to recover extended emission. Data reduction was done with the BOA software (P. Schuller et al., in preparation) following standard procedures, including iterative source modeling. SABOCA (Siringo et al. 2010) is a 37 bolometer array operating at $350 \mu \mathrm{m}$, with a resolution of $\sim 7^{\prime \prime} .3 \mathrm{FWHM}$. The observing and data reduction procedures were similar to those used for LABOCA. For both cameras, observations were carried out between 2009 November and 2012 June, and are still ongoing to complete our Submillimeter Orion Survey. Conditions were generally fair over the course of our observing campaign. The observations will be summarized in more detail by T. Stanke et al. (2013, in preparation).

The beam sizes of the final reduced maps are 7.34 and 19.'0 FWHM for the SABOCA and LABOCA observations, respectively. The photometry was extracted in the same way for both wavelengths. When possible, if there was a strong source detection, we re-centered using the $70 \mu \mathrm{m}$ catalog source coordinates. Given the contributions of flux due to surrounding cold material, such as filaments and other extended envelope structure, it is likely that a single photometric measure can suffer from large systematic effects. We have measured source fluxes in three ways.

1. We measured the source peak flux per beam.

2. We measured source flux over an aperture with radius equal to the FWHM at the corresponding wavelength 
Table 1

Summary of HOPS Herschel PACS L1641 Observations

\begin{tabular}{|c|c|c|c|c|c|c|}
\hline $\begin{array}{l}\text { HOPS } \\
\text { Group Name }\end{array}$ & $\begin{array}{l}\text { R.A. }^{\mathrm{a}} \\
\text { (h:m:s) }\end{array}$ & $\begin{array}{l}\text { Decl. }{ }^{\mathrm{a}} \\
\left({ }^{\circ}:^{\prime} ;^{\prime \prime}\right)\end{array}$ & Field & AOR ID & $\mathrm{OD}$ & $\begin{array}{c}\text { Map Size } \\
\left({ }^{\prime} \times{ }^{\prime}\right)\end{array}$ \\
\hline 54 & $5: 42: 38.570$ & $-8: 50: 18.67$ & L1641 & $1342218796(7)$ & 704 & $8 \times 8$ \\
\hline 53 & 5:43:06.770 & $-8: 46: 09.56$ & L1641 & $1342218735(6)$ & 703 & $8 \times 8$ \\
\hline 60 & $5: 41: 29.690$ & $-8: 41: 28.59$ & L1641 & $1342215359(60)$ & 662 & $8 \times 8$ \\
\hline 55 & $5: 42: 50.490$ & $-8: 40: 54.73$ & L1641 & $1342218798(9)$ & 704 & $8 \times 8$ \\
\hline 56 & $5: 42: 52.750$ & $-8: 37: 20.98$ & L1641 & $1342205256(7)$ & 502 & $8 \times 8$ \\
\hline 117 & $5: 41: 33.250$ & $-8: 36: 41.62$ & L1641 & $1342218790(1)$ & 704 & $8 \times 8$ \\
\hline 59 & $5: 42: 55.540$ & $-8: 32: 48.26$ & L1641 & $1342218794(5)$ & 704 & $8 \times 8$ \\
\hline 58 & 5:43:09.580 & $-8: 29: 27.13$ & L1641 & $1342218788(9)$ & 704 & $8 \times 8$ \\
\hline 61 & $5: 42: 47.760$ & $-8: 16: 50.72$ & L1641 & $1342205254(5)$ & 502 & $8 \times 8$ \\
\hline 67 & $5: 40: 20.130$ & $-8: 14: 05.78$ & L1641 & 1342227078(9) & 831 & $8 \times 8$ \\
\hline 119 & $5: 40: 47.820$ & $-8: 10: 28.38$ & L1641 & $1342206322(3)$ & 516 & $8 \times 8$ \\
\hline 62 & $5: 42: 47.370$ & $-8: 10: 08.76$ & L1641 & 1342218792(3) & 704 & $8 \times 8$ \\
\hline 63 & $5: 41: 35.440$ & $-8: 08: 22.49$ & L1641 & $1342218800(1)$ & 704 & $8 \times 8$ \\
\hline 66 & $5: 40: 54.500$ & $-8: 06: 08.98$ & L1641 & 1342215361(2) & 662 & $8 \times 8$ \\
\hline 118 & $5: 41: 27.790$ & $-8: 04: 03.70$ & L1641 & $1342205250(1)$ & 502 & $8 \times 8$ \\
\hline 78 & 5:38:51.480 & $-8: 01: 27.44$ & L1641 & $1342228169(70)$ & 844 & $5 \times 5$ \\
\hline 64 & $5: 41: 49.950$ & $-8: 01: 26.51$ & L1641 & $1342205252(3)$ & 502 & $5 \times 5$ \\
\hline 69 & $5: 40: 38.330$ & $-8: 00: 36.00$ & L1641 & $1342227080(1)$ & 831 & $5 \times 5$ \\
\hline 65 & $5: 41: 24.880$ & $-8: 00: 02.34$ & L1641 & $1342215591(2)$ & 663 & $8 \times 8$ \\
\hline 72 & $5: 40: 20.710$ & $-7: 56: 01.36$ & L1641 & $1342218733(4)$ & 703 & $8 \times 8$ \\
\hline 70 & $5: 40: 40.530$ & $-7: 54: 39.82$ & L1641 & $1342228167(8)$ & 844 & $5 \times 5$ \\
\hline 121 & $5: 41: 23.440$ & $-7: 54: 39.26$ & L1641 & $1342205248(9)$ & 502 & $8 \times 8$ \\
\hline 68 & $5: 41: 19.660$ & $-7: 50: 41.03$ & L1641 & $1342227848(9)$ & 842 & $5 \times 5$ \\
\hline 74 & $5: 40: 17.690$ & $-7: 49: 29.88$ & L1641 & $1342218731(2)$ & 703 & $8 \times 8$ \\
\hline 71 & $5: 40: 40.150$ & $-7: 49: 18.71$ & L1641 & $1342228163(4)$ & 844 & $8 \times 8$ \\
\hline 320 & $5: 40: 58.890$ & $-7: 48: 02.05$ & L1641 & $1342228165(6)$ & 844 & $5 \times 5$ \\
\hline 73 & $5: 40: 42.910$ & $-7: 45: 01.91$ & L1641 & $1342228425(6)$ & 847 & $5 \times 5$ \\
\hline 75 & $5: 40: 24.620$ & $-7: 43: 08.26$ & L1641 & $1342227082(3)$ & 831 & $5 \times 5$ \\
\hline 76 & $5: 40: 26.090$ & $-7: 37: 32.02$ & L1641 & $1342205246(7)$ & 502 & $5 \times 5$ \\
\hline 79 & 5:39:57.200 & $-7: 30: 19.89$ & L1641 & $1342205244(5)$ & 502 & $8 \times 8$ \\
\hline 77 & $5: 40: 44.670$ & $-7: 29: 54.46$ & L1641 & $1342228427(8)$ & 847 & $5 \times 5$ \\
\hline 123 & 5:40:08.780 & $-7: 27: 27.68$ & L1641 & 1342228161(2) & 844 & $5 \times 5$ \\
\hline 26 & $5: 39: 24.640$ & $-7: 26: 13.81$ & L1641 & $1342218729(30)$ & 703 & $8 \times 8$ \\
\hline 25 & 5:39:56.200 & $-7: 24: 53.71$ & L1641 & $1342215589(90)$ & 663 & $8 \times 8$ \\
\hline 313 & $5: 39: 33.300$ & $-7: 22: 57.36$ & L1641 & $1342227084(5)$ & 831 & $5 \times 5$ \\
\hline 28 & $5: 38: 56.470$ & $-7: 20: 44.32$ & L1641 & $1342227086(7)$ & 831 & $8 \times 8$ \\
\hline 30 & $5: 38: 44.050$ & $-7: 11: 49.89$ & L1641 & $1342204254(5)$ & 484 & $8 \times 8$ \\
\hline 29 & 5:39:06.710 & $-7: 11: 12.80$ & L1641 & $1342204252(3)$ & 484 & $8 \times 8$ \\
\hline 32 & 5:38:01.100 & $-7: 07: 37.01$ & L1641 & $1342227045(6)$ & 830 & $8 \times 8$ \\
\hline 312 & $5: 38: 46.540$ & $-7: 05: 37.46$ & L1641 & $1342205242(3)$ & 502 & $5 \times 5$ \\
\hline 31 & $5: 38: 44.870$ & $-7: 00: 37.03$ & L1641 & $1342204256(7)$ & 484 & $8 \times 8$ \\
\hline 33 & $5: 38: 20.090$ & $-6: 59: 04.85$ & L1641 & $1342228171(2)$ & 844 & $5 \times 5$ \\
\hline 35 & $5: 37: 24.460$ & $-6: 58: 32.77$ & L1641 & $1342227314(5)$ & 833 & $5 \times 5$ \\
\hline 34 & $5: 37: 59.990$ & $-6: 57: 27.50$ & L1641 & $1342205240(1)$ & 502 & $8 \times 8$ \\
\hline 101 & $5: 37: 17.090$ & $-6: 49: 49.33$ & L1641 & $1342227312(3)$ & 833 & $5 \times 5$ \\
\hline 36 & $5: 37: 52.390$ & $-6: 47: 18.67$ & L1641 & 1342227088(9) & 831 & $8 \times 8$ \\
\hline 38 & $5: 36: 22.050$ & $-6: 45: 41.23$ & L1641 & $1342205238(9)$ & 502 & $8 \times 8$ \\
\hline 40 & $5: 36: 26.650$ & $-6: 38: 27.74$ & L1641 & $1342227094(5)$ & 831 & $8 \times 8$ \\
\hline 37 & $5: 37: 17.280$ & $-6: 36: 18.18$ & L1641 & $1342227090(1)$ & 831 & $8 \times 8$ \\
\hline 43 & $5: 35: 50.020$ & $-6: 34: 53.40$ & L1641 & $1342227310(1)$ & 833 & $5 \times 5$ \\
\hline 50 & $5: 34: 15.880$ & $-6: 34: 32.70$ & L1641 & $1342217748(9)$ & 686 & $8 \times 8$ \\
\hline 39 & $5: 36: 41.330$ & $-6: 34: 00.08$ & L1641 & $1342227092(3)$ & 831 & $5 \times 5$ \\
\hline 41 & $5: 36: 19.440$ & $-6: 29: 06.79$ & L1641 & $1342227316(7)$ & 833 & $5 \times 5$ \\
\hline 45 & $5: 35: 34.120$ & $-6: 26: 41.70$ & L1641 & $1342215593(4)$ & 663 & $8 \times 8$ \\
\hline 311 & $5: 34: 39.860$ & $-6: 25: 14.16$ & L1641 & $1342203649(50)$ & 470 & $5 \times 5$ \\
\hline 42 & $5: 36: 22.460$ & $-6: 23: 39.14$ & L1641 & $1342205236(7)$ & 502 & $8 \times 8$ \\
\hline 44 & $5: 36: 36.980$ & $-6: 14: 57.98$ & L1641 & $1342204258(9)$ & 484 & $5 \times 5$ \\
\hline 51 & $5: 35: 22.180$ & $-6: 13: 06.24$ & L1641 & $1342227318(9)$ & 833 & $5 \times 5$ \\
\hline 47 & $5: 36: 17.260$ & $-6: 11: 11.00$ & L1641 & $1342227324(5)$ & 833 & $5 \times 5$ \\
\hline 49 & $5: 35: 52.000$ & $-6: 10: 01.85$ & L1641 & $1342227322(3)$ & 833 & $5 \times 5$ \\
\hline 52 & $5: 35: 33.210$ & $-6: 06: 09.65$ & L1641 & $1342227320(1)$ & 833 & $5 \times 5$ \\
\hline 48 & $5: 36: 31.360$ & $-6: 01: 16.81$ & L1641 & $1342217444(5)$ & 685 & $8 \times 8$ \\
\hline 5 & 5:35:07.960 & $-5: 56: 56.40$ & L1641 & $1342204248(9)$ & 484 & $8 \times 8$ \\
\hline 6 & $5: 35: 24.560$ & $-5: 55: 33.42$ & L1641 & $1342227328(9)$ & 833 & $5 \times 5$ \\
\hline
\end{tabular}


Table 1

(Continued)

\begin{tabular}{lcccccc}
\hline \hline $\begin{array}{l}\text { HOPS } \\
\text { Group Name }\end{array}$ & $\begin{array}{c}\text { R.A. }^{\text {a }} \\
\text { (h:m:s })\end{array}$ & $\begin{array}{c}\text { Decl. } \\
\left({ }^{\circ}:^{\prime} ; ;^{\prime \prime}\right.\end{array}$ & Field & AOR ID & OD & $\begin{array}{c}\text { Map Size } \\
\left({ }^{\prime} \times{ }^{\prime}\right)\end{array}$ \\
\hline 7 & $5: 36: 19.020$ & $-5: 55: 25.46$ & L1641 & $1342227326(7)$ & 833 & $5 \times 5$ \\
8 & $5: 35: 04.400$ & $-5: 51: 00.76$ & L1641 & $1342217446(7)$ & 685 & $8 \times 8$ \\
9 & $5: 33: 30.710$ & $-5: 50: 41.03$ & L1641 & $1342217750(1)$ & 686 & $8 \times 8$ \\
10 & $5: 36: 10.100$ & $-5: 50: 08.34$ & L1641 & $1342227096(7)$ & 831 & $5 \times 5$ \\
12 & $5: 34: 46.830$ & $-5: 42: 28.72$ & L1641 & $1342204246(7)$ & 484 & $8 \times 8$ \\
13 & $5: 35: 17.340$ & $-5: 42: 14.51$ & L1641 & $1342227098(9)$ & 831 & $5 \times 5$ \\
14 & $5: 34: 30.440$ & $-5: 37: 47.44$ & L1641 & $1342204244(5)$ & 484 & $8 \times 8$ \\
\hline
\end{tabular}

Note. ${ }^{\text {a }}$ Field center coordinates.

Table 2

Summary of HOPS Herschel PACS ONC, NGC 2024, NGC 2068, and L1622 Observations

\begin{tabular}{|c|c|c|c|c|c|c|}
\hline $\begin{array}{l}\text { HOPS } \\
\text { Group Name }\end{array}$ & $\begin{array}{l}\text { R.A. }^{\mathrm{a}} \\
\text { (h:m:s) }\end{array}$ & $\begin{array}{l}\text { Decl. }{ }^{\mathrm{a}} \\
\left({ }^{\circ}:^{\prime} ; ;^{\prime \prime}\right)\end{array}$ & Region & AOR ID & $\mathrm{OD}$ & $\begin{array}{c}\text { Map Size } \\
\left({ }^{\prime} \times{ }^{\prime}\right)\end{array}$ \\
\hline 15 & $5: 35: 06.620$ & $-5: 35: 05.68$ & ONC & $1342205234(5)$ & 502 & $8 \times 8$ \\
\hline 308 & $5: 33: 45.870$ & $-5: 32: 58.09$ & ONC & $1342204433(4)$ & 487 & $5 \times 5$ \\
\hline 16 & $5: 34: 43.990$ & $-5: 32: 11.21$ & ONC & $1342217448(9)$ & 685 & $8 \times 8$ \\
\hline 17 & $5: 35: 16.320$ & $-5: 29: 32.60$ & ONC & $1342217450(1)$ & 685 & $8 \times 8$ \\
\hline 18 & $5: 33: 55.730$ & $-5: 22: 39.97$ & ONC & $1342217752(3)$ & 686 & $8 \times 8$ \\
\hline 200 & $5: 35: 19.270$ & $-5: 14: 46.49$ & ONC & $1342205232(3)$ & 502 & $8 \times 8$ \\
\hline 130 & $5: 35: 24.710$ & $-5: 09: 06.02$ & ONC & $1342205228(9)$ & 502 & $8 \times 8$ \\
\hline 135 & $5: 35: 26.280$ & $-5: 06: 35.24$ & ONC & $1342205226(7)$ & 502 & $8 \times 8$ \\
\hline 19 & $5: 35: 23.300$ & $-5: 00: 35.73$ & ONC & $1342204250(1)$ & 484 & $8 \times 8$ \\
\hline 20 & $5: 35: 13.770$ & $-4: 54: 57.33$ & ONC & $1342217758(9)$ & 686 & $8 \times 8$ \\
\hline 21 & $5: 34: 32.340$ & $-4: 53: 54.26$ & ONC & $1342217754(5)$ & 686 & $8 \times 8$ \\
\hline 306 & $5: 35: 32.280$ & $-4: 46: 48.47$ & ONC & $1342191970(1)$ & 300 & $5 \times 5$ \\
\hline 24 & $5: 35: 23.340$ & $-4: 40: 10.45$ & ONC & $1342217756(7)$ & 686 & $8 \times 8$ \\
\hline 80 & $5: 40: 51.710$ & $-2: 26: 48.62$ & NGC 2024 & $1342226729(30)$ & 826 & $5 \times 5$ \\
\hline 81 & $5: 41: 28.940$ & $-2: 23: 19.36$ & NGC 2024 & $1342226733(4)$ & 826 & $5 \times 5$ \\
\hline 82 & $5: 41: 23.740$ & $-2: 16: 51.10$ & NGC 2024 & $1342228913(4)$ & 858 & $8 \times 8$ \\
\hline 83 & $5: 41: 42.180$ & $-2: 16: 26.20$ & NGC 2024 & $1342227049(50)$ & 830 & $8 \times 8$ \\
\hline 85 & $5: 42: 02.620$ & $-2: 07: 45.70$ & NGC 2024 & $1342226735(6)$ & 826 & $5 \times 5$ \\
\hline 86 & $5: 41: 43.560$ & $-1: 53: 28.42$ & NGC 2024 & $1342227047(8)$ & 830 & $8 \times 8$ \\
\hline 89 & $5: 42: 27.680$ & $-1: 20: 01.00$ & NGC 2024 & $1342205220(1)$ & 502 & $5 \times 5$ \\
\hline 90 & $5: 43: 04.370$ & $-1: 16: 11.60$ & NGC 2024 & $1342228376(7)$ & 849 & $8 \times 8$ \\
\hline 91 & $5: 46: 06.690$ & $-0: 13: 05.15$ & NGC 2068 & $1342205218(9)$ & 502 & $8 \times 8$ \\
\hline 92 & $5: 46: 14.210$ & $-0: 05: 26.84$ & NGC 2068 & $1342205216(7)$ & 502 & $5 \times 5$ \\
\hline 93 & $5: 46: 40.830$ & $+0: 00: 30.52$ & NGC 2068 & $1342215363(4)$ & 662 & $8 \times 8$ \\
\hline 94 & $5: 46: 39.580$ & $+0: 04: 16.61$ & NGC 2068 & $1342228365(6)$ & 848 & $5 \times 5$ \\
\hline 302 & $5: 46: 28.320$ & $+0: 19: 49.40$ & NGC 2068 & $1342228374(5)$ & 849 & $5 \times 5$ \\
\hline 303 & $5: 47: 24.810$ & $+0: 20: 59.68$ & NGC 2068 & $1342227966(7)$ & 843 & $8 \times 8$ \\
\hline 96 & $5: 47: 08.970$ & $+0: 21: 52.86$ & NGC 2068 & $1342215587(8)$ & 663 & $8 \times 8$ \\
\hline 128 & $5: 46: 56.220$ & $+0: 23: 42.41$ & NGC 2068 & $1342218727(8)$ & 703 & $8 \times 8$ \\
\hline 301 & $5: 45: 53.590$ & $+0: 25: 27.30$ & NGC 2068 & $1342216450(1)$ & 675 & $5 \times 5$ \\
\hline 97 & $5: 47: 58.060$ & $+0: 35: 30.12$ & NGC 2068 & $1342227969(70)$ & 843 & $8 \times 8$ \\
\hline 98 & $5: 47: 31.850$ & $+0: 38: 05.77$ & NGC 2068 & 1342227971(2) & 843 & $8 \times 8$ \\
\hline 300 & $5: 47: 42.990$ & $+0: 40: 57.50$ & NGC 2068 & $1342205214(5)$ & 502 & $5 \times 5$ \\
\hline 0 & $5: 54: 15.240$ & $+1: 43: 15.59$ & L1622 & $1342215365(6)$ & 662 & $8 \times 8$ \\
\hline 1 & $5: 54: 55.370$ & $+1: 45: 03.08$ & L1622 & $1342218780(1)$ & 704 & $8 \times 8$ \\
\hline 3 & $5: 54: 23.540$ & $+1: 49: 17.78$ & L1622 & $1342218703(4)$ & 702 & $8 \times 8$ \\
\hline 4 & $5: 54: 36.260$ & $+1: 53: 54.00$ & L1622 & $1342218778(9)$ & 704 & $8 \times 8$ \\
\hline
\end{tabular}

Notes. The ONC field contains the extended Orion Nebula region, the NGC 1977 region, and OMC2/3; the NGC 2024 field contains the NGC 2024 H II region and the NGC 2023 reflection nebula. The NGC 2068 field includes the NGC 2068 and NGC 2071 reflection nebulae as well as LBS23 region.

${ }^{\text {a }}$ Field center coordinates.

$\left(r=7^{\prime \prime} .34\right.$ and $19 . \prime 0$ at 350 and $870 \mu \mathrm{m}$, respectively), using a sky annulus with inner and outer radii equal to $[1.5,2.0] \times \mathrm{FWHM}$, corresponding to $11^{\prime \prime} .0$ and $14^{\prime \prime} .7$ at $350 \mu \mathrm{m}$, and $28^{\prime \prime} .5$ and $38^{\prime \prime} .0$ at $870 \mu \mathrm{m}$.
3. We measured the flux over the same aperture size as the previous method without any sky subtraction. In the case where a source was not strongly detected and we were not able to re-center, the $70 \mu \mathrm{m}$ catalog source 
coordinates were used, along with method 3, and the photometric point was flagged as an upper limit. By recentering whenever possible, we accounted for possible pointing offsets between data sets, which can be significant. The calibration error dominated the error budget for welldetected sources; we therefore adopted a flux error equal to $20 \%$ and $40 \%$ of the measured flux for LABOCA and SABOCA, respectively. The photometric fluxes are presented in Table 5.

\subsection{Spitzer IRAC and MIPS}

The IRAC and MIPS imaging and photometry presented here are taken from the $9 \mathrm{deg}^{2}$ survey of the Orion A and B cloud obtained during the cryogenic Spitzer mission. The data analysis, extraction of the IRAC $3.6 \mu \mathrm{m}, 4.5 \mu \mathrm{m}, 5.8 \mu \mathrm{m}$, and $8 \mu \mathrm{m}$ and MIPS $24 \mu \mathrm{m}$ photometry, and the compilation of a point-source catalog containing the combined Two Micron All Sky Survey (2MASS), IRAC, and MIPS photometry are described in Megeath et al. (2012); see also Kryukova et al. (2012) for a detailed description of the MIPS $24 \mu \mathrm{m}$ photometry. In total, 298,405 point sources were detected in at least one of the Spitzer bands, and 8021 sources were detected at $24 \mu \mathrm{m}$ with uncertainties $\leqslant 0.25 \mathrm{mag}$. The Spitzer images used in this work are taken from the mosaics generated from the Orion Survey data using Cluster Grinder for the IRAC data (Gutermuth et al. 2009) and the MIPS instrument team's Data Analysis Tool for the $24 \mu \mathrm{m}$ data (Gordon et al. 2005). The MIPS data are saturated toward the Orion Nebula and parts of the NGC 2024 region; we exclude these saturated regions from our analysis.

The identification of protostars with the Spitzer data was based primarily on the presence of a flat or rising SED between $4.5 \mu \mathrm{m}$ and $24 \mu \mathrm{m}$ (Kryukova et al. 2012; Megeath et al. 2012). In addition, Megeath et al. identified objects which have pointsource detections only at $24 \mu \mathrm{m}$ but which also showed other indicators of protostellar nature such as the presence of jets in the IRAC bands. To minimize contamination from galaxies, the Spitzer-identified protostars were required to have $24 \mu \mathrm{m}$ magnitudes brighter than 7 th magnitude; fainter than 7 mag, the number of background galaxies begins to dominate over the number of embedded sources (Kryukova et al. 2012). Given the imposed $24 \mu \mathrm{m}$ magnitude threshold, the faintest and reddest protostars may not be included in the Spitzer sample. In total, the Megeath et al. (2012) catalog contains 488 protostars. Of these, 428 are classified as bona fide protostars, 50 are faint candidate protostars, and 10 are red candidate protostars. The faint candidate protostars are sources with $24 \mu \mathrm{m}$ magnitudes higher than 7.0. The red protostars are sources that are only detected as a point source at $24 \mu \mathrm{m}$ and are thus not classifiable. Due to their location within high extinction regions and/or association with jets or compact scattered light nebulae in the IRAC bands, they have been included in the catalog. (Indeed, this last category was added to the Megeath et al. catalog after the Herschel data revealed that a relatively large number of such sources would likely be confirmed as protostars.) In addition, the Megeath et al. catalog identified 2992 objects as pre-mainsequence stars with disks. In what follows, we use the protostar catalog of 488 Spitzer sources to catalog previously identified sources in the HOPS images.

In contrast to the full Megeath et al. catalog, the HOPS protostar sample is composed of protostars specifically targeted by the HOPS program. The majority of the HOPS sample consists of Spitzer-identified protostars with $24 \mu \mathrm{m}$ detections; hence, protostars in regions that are saturated in the $24 \mu \mathrm{m}$ images of Orion, namely the brightest regions of the Orion Nebula and NGC 2024, are not included. These protostars were also required to have a predicted $70 \mu \mathrm{m}$ flux $>20 \mathrm{mJy}$ as extrapolated from their $3 \mu \mathrm{m}$ to $24 \mu \mathrm{m}$ SEDs. In addition, protostar candidates with only $24 \mu \mathrm{m}$ detections were included if there was independent information of their protostellar nature. The HOPS sample represents the best pre-Herschel catalog of protostars that were expected to be detected with Herschel/ PACS and were not found in bright nebulous regions. There are $\sim 300$ protostars in the HOPS catalog which have been detected at $70 \mu \mathrm{m}$ and $\sim 250$ protostars detected at $160 \mu \mathrm{m}$. The uncertainty in the absolute number of protostars is due to the ongoing process of eliminating contamination from the sample.

\section{IDENTIFICATION OF NEW CANDIDATE HERSCHEL PROTOSTARS}

To find protostars which were not reliably identified with Spitzer, we must first isolate a sample of sources that are detected in the PACS $70 \mu \mathrm{m}$ band but are either fainter than 7.0 mag or undetected in MIPS $24 \mu \mathrm{m}$ waveband. To identify such sources in each HOPS field, we first generate a $70 \mu \mathrm{m}$ source catalog using the PhotVis tool (Gutermuth et al. 2008). The PhotVis tool uses a sunken Gaussian filtering to extract sources that are of order the size of the Gaussian FWHM, an input parameter. We choose this parameter to be the size of the $70 \mu \mathrm{m}$ PSF FWHM, or $5^{\prime \prime}$. PhotVis also requires a signal-to-noise ratio threshold as input; we adopt a low value of 7 to balance the recovery of as many candidate sources as possible while still rejecting noise spikes.

Furthermore, we must reject unreliable sources near the edges of maps where the lower coverage causes elevated noise levels. The Scanamorphos scan-map image cubes include a weight map for each field. Within Scanamorphos, the weight map is computed over the same projection as the sky map, and is defined as 1 over the variance in the white noise (Roussel 2012). Each weight map is then normalized by the average map value (Roussel 2012). We find that for the HOPS data set, weight map values of $\sim 20$ are confined to the outer higher noise edges of our scan maps. We therefore use the weight maps to reject edge sources from the catalog at this phase of the analysis. We accomplish this by requiring that the mean value of the weight map in a $9 \times 9$ pixel area centered on the candidate source has a value of at least 20. For reference, all HOPS $70 \mu \mathrm{m}$ scan maps have weight map values greater than 60 over most of the map areas.

The resulting preliminary source catalog includes all sources in the $70 \mu \mathrm{m}$ images, i.e., previously identified Spitzer sources, new candidate protostars, nebulosity, and other undesirable features and artifacts in the images. We then cross-correlate this PACS $70 \mu \mathrm{m}$ preliminary catalog with the existing Spitzer catalog to eliminate all previously identified protostars in each field that are brighter than the previously adopted $24 \mu \mathrm{m}$ cutoff of 7 mag (Megeath et al. 2012). Therefore, our sample includes by definition only sources that are faint or undetected in the previous Spitzer catalog.

To identify previous source detections, we require that a source be matched to within a positional offset of $8^{\prime \prime}$ when cross-correlated with the Spitzer catalog. This threshold is conservatively large compared to the Spitzer astrometry and is meant to encompass two main sources of astrometric error. First, it is possible that the absolute coordinates of a source may shift as a function of wavelength (although this effect is expected to be 


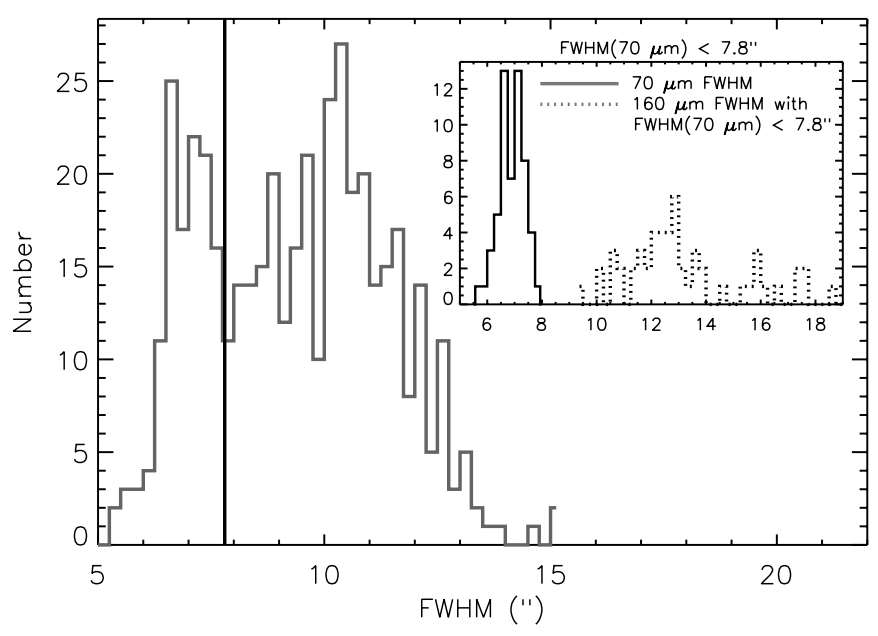

Figure 1. Frequency of $70 \mu \mathrm{m}$ FWHM values for all sources detected in our HOPS images that have not been previously identified in the Spitzer catalog. The black line shows our adopted 7".8 FWHM threshold, above which we reject all sources. Inset: the same as above for the subset of sources with both FWHM $(70 \mu \mathrm{m})<7$ ". 8 and $160 \mu \mathrm{m}$ detections (see Section 3). The dotted histogram indicates the $160 \mu \mathrm{m}$ FWHM distribution.

relatively small) since different wavelengths may trace different material near the protostars. Second, the Herschel pointing accuracy, which is of order $\sim 2^{\prime \prime}(1 \sigma)$, dominates the positional uncertainty for most sources when comparing the Spitzer catalog source coordinates to the Herschel $70 \mu \mathrm{m}$ coordinates. To match coordinates robustly, we therefore adopt a conservatively large $8^{\prime \prime}$ threshold. We find that inspection of the matched sources by eye shows that this threshold works well and provides a low rate of mismatched or duplicate sources.

Our final goal is to obtain a sample of previously unidentified and uncharacterized Herschel protostar candidates. These sources should be characterized by a point-like appearance at $70 \mu \mathrm{m}$. Therefore, after rejecting all Spitzer protostars as described above, we next apply a simple FWHM (or apparent size) filter to the remaining $70 \mu \mathrm{m}$ sources. The distribution of $70 \mu \mathrm{m}$ azimuthally averaged FWHM values is shown in Figure 1 as the solid black histogram. We find a clear peak in the distribution at low FWHM values, indicating a population of point-like sources. Based on this distribution, we adopt an FWHM threshold of 7".8, meant to select $70 \mu \mathrm{m}$ point sources. We find 127 sources that fulfill the criteria listed here: 85 of these have $24 \mu \mathrm{m}$ detections while 42 do not.

In a further step, we then require that all $70 \mu \mathrm{m}$ sources also have a $160 \mu \mathrm{m}$ detection and not upper limits. The $70 \mu \mathrm{m}$ FWHM distribution of this subset of sources is shown in the inset of Figure 1 along with the corresponding $160 \mu \mathrm{m}$ FWHM distribution. Our final sample consists of 55 candidate Herschel protostars with both $70 \mu \mathrm{m}$ and $160 \mu \mathrm{m}$ detections. Of these, 34 have Spitzer $24 \mu \mathrm{m}$ detections fainter than $7.0 \mathrm{mag}$ and 21 do not have any $24 \mu \mathrm{m}$ detection.

\subsection{Spitzer Non-detections}

A search for newly detected protostars using Herschel requires us to determine upper limits at $24 \mu \mathrm{m}$ for those sources that are not detected by Spitzer. To determine these limits, we adopt the method developed by Megeath et al. to assess the spatially varying completeness of the Spitzer Orion Survey data. The completeness of the $24 \mu \mathrm{m}$ data depends strongly on the presence of nebulosity and point-source crowding. To account for these factors, we measure the fluctuations of the $24 \mu \mathrm{m}$ sig- nal in an annulus centered on the position of the Herschel point source using the root median square deviation, or RMEDSQ (see Equation (1) of Megeath et al. 2012). We then use the results from the artificial star tests (see the Appendix of Megeath et al. 2012) to determine the magnitude at which $90 \%$ of the point sources would be detected for the observed level of fluctuations. We convert this magnitude into a flux density to obtain $24 \mu \mathrm{m}$ upper limits.

Several of the identified protostars show IRAC emission but are not included in the Megeath et al. point-source catalog because they are spatially extended. To obtain homogeneously extracted IRAC fluxes for the entire sample of sources, we measure fluxes using an aperture of 2 pixels, with a sky annulus of 2-6 pixels, corresponding to radii of 2 ".44, 2".44, and 7".33, respectively, with a pixel scale of $1^{\prime \prime} .22$ pixel $^{-1}$. We use the PACS $70 \mu \mathrm{m}$ source coordinates as starting guesses, and attempt to recenter at each IRAC wavelength. If the re-centering fails, as for sources with no IRAC detections, we take the integrated flux in that aperture at the original PACS $70 \mu \mathrm{m}$ source coordinate to be the upper limit. The aperture corrections and photometric zero points are those given by Kryukova et al.

\subsection{Contamination in the Sample}

Galaxies often exhibit infrared colors similar to those of young stellar objects (YSOs) due to the presence of dust and hydrocarbons in the galaxies (Stern et al. 2005). Extensive work has been done toward characterizing the extragalactic "contamination" in Spitzer surveys of star-forming regions and mitigating it through photometric criteria designed to separate galaxies from bona fide YSOs (Gutermuth et al. 2009, 2008; Harvey et al. 2007). These authors show that star-forming galaxies can be distinguished from YSOs by the galaxies' stellar-like emission in the IRAC $3.6 \mu \mathrm{m}$ and $4.5 \mu \mathrm{m}$ bands and their bright polycyclic aromatic hydrocarbon (PAH) emission in the IRAC $5.8 \mu \mathrm{m}$ and $8.0 \mu \mathrm{m}$ bands (Gutermuth et al. 2009, 2008; Winston et al. 2007; Stern et al. 2005). However, we note that some active galactic nuclei (AGNs) dominated galaxies may not exhibit PAH emission; therefore, an analysis based only on the IRAC colors may not capture all possible sources of contamination (Robitaille et al. 2008).

To analyze the IRAC colors of our sample, we define $\alpha=d \log \left(\lambda F_{\lambda}\right) / d \log (\lambda)$. In Figure 2, we plot $\alpha_{5.8-8}$ versus $\alpha_{3.6-4.5}$ for the sample of new Herschel sources with coverage in all four of the Spitzer/IRAC bands and detections in at least one band, compared to the HOPS protostar sample. This color index is relatively insensitive to reddening since the extinction in the $5.8 \mu \mathrm{m}$ and $8 \mu \mathrm{m}$ bands of IRAC are very similar (e.g., Flaherty et al. 2007; Gutermuth et al. 2008). Figure 2 shows a cluster of sources with high values of $\alpha_{5.8-8}$ (i.e., $\alpha_{5.8-8} \geqslant 3$; solid horizontal line) yet $\alpha_{3.6-4.5}$ values of an SED that is declining or flat with increasing wavelength. These sources show the characteristics of star-forming galaxies with bright PAH emission (resulting in high values of $\alpha_{5.8-8}$ ) but values of $\alpha_{3.6-4.5}$ that are dominated by starlight. In our adopted scheme, $\alpha_{5.8-8} \geqslant 3$ corresponds to a color of [5.8] $-[8] \geqslant 2.17$; this threshold is higher than the [5.8] $-[8] \geqslant 1$ threshold used by Gutermuth et al. (2008) to isolate galaxies and thus ensures that most protostellar candidates will be less likely to be misidentified extragalactic sources (Allen et al. 2004; Megeath et al. 2004). We identify the cluster of sources with $\alpha_{5.8-8} \geqslant 3$ and $\alpha_{3.6-4.5} \leqslant 0.5$ as likely extragalactic contamination. We note that nebular contamination of the photometry can cause PAH-like $\alpha_{5.8-8}$ values, and thus may 


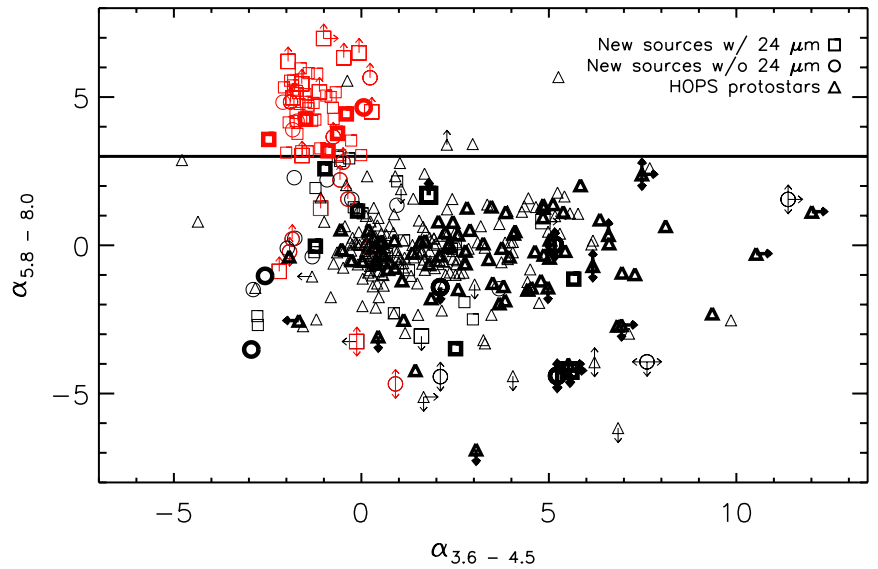

Figure 2. IRAC color indices $\alpha_{3.6-4.5}$ and $\alpha_{5.8-8.0}$ for sources detected in at least one IRAC band. The Herschel protostar candidate sample is shown as squares (sources with $24 \mu \mathrm{m}$ detections) and circles (sources without $24 \mu \mathrm{m}$ detections), while the HOPS protostar sample is shown as triangles. Thick bold points are sources with robust $870 \mu \mathrm{m}$ detections. Candidate protostars with $\alpha_{5.8-8.0}>3.0$ or upper limits consistent with this threshold are flagged as possible extragalactic contamination and highlighted in red. Note that some candidate protostars have both $\alpha_{5.8-8.0}>3.0$ and a robust $870 \mu \mathrm{m}$ detection; these sources are not considered extragalactic contamination.

(A color version of this figure is available in the online journal.)

cause us to overestimate the extragalactic contamination. Of the 55 sources identified here as protostellar candidates, we flag 23 as possible extragalactic contamination based on this criterion. However, other sources of contamination, such as AGNs lacking $\mathrm{PAH}$ emission, may remain in our sample.

Inspection of the SEDs of the remaining 32 sources shows a range of SED slopes and shapes. It is possible that such sources may also be extragalactic contamination by AGN, for example. To address this, we further refine the analysis presented above by analyzing the $3.6 \mu \mathrm{m}$ to $160 \mu \mathrm{m}$ SED shapes with the spectral index $\alpha_{3.6-160}$. As illustrated in Figure 3, the sources flagged as extragalactic based on the $\alpha_{5.8-8}$ index (red points) generally have $\alpha_{3.6-160} \lesssim 1.2$. We therefore calibrate the $\alpha_{3.6-160}$ relative to the reliable extragalactic candidates with robust IRAC detections and expect that extragalactic sources will have $\alpha_{3.6-160} \lesssim 1.2$. Using this criterion, we refine our source classification as follows. All sources with $\alpha_{3.6-160}>1.2$ (and $\alpha_{5.8-8}<3$ when IRAC detections exist) are flagged as high probability protostars (flag $=1$ in Table 3 ). Sources having values of $0.5 \lesssim \alpha_{3.6-160} \lesssim 1.2$ but that were originally classified as candidate protostars based on a low value of $\alpha_{5.8-8}$ are flagged as less likely to be of a protostellar nature (flag $=2$ in Table 3). Furthermore, by definition, sources originally classified as extragalactic based on their PAH signature at $8 \mu \mathrm{m}$ remain classified as such (flag $=3$ in Table 3). Sources with $\alpha_{3.6-160}<0.3$ and $\alpha_{3.6-4.5} \sim-3$ are flagged as "other" (flag $=$ 4 in Table 3) since their SEDs are consistent with a stellar photosphere at shorter wavelengths. Finally, one source has no IRAC coverage and therefore is flagged with a value of 5 . In Figure 4, we show example SEDs of each category.

Only one source (313006) originally flagged as extragalactic based on its $\alpha_{5.8-8}$ limit (non-detection at $5.8 \mu \mathrm{m}$ and $8 \mu \mathrm{m}$ ) was revised to a highly probable protostar (see Figure 3 and the top left panel of Figure 4). In addition, as we note above, we find three sources with SEDs that we label "other" (flag value =4) which are inconsistent with the categories described above. Source 069001 (see Figure 4, top right panel) was previously characterized by Fang et al. (2009) as a K7 star with a debris disk,

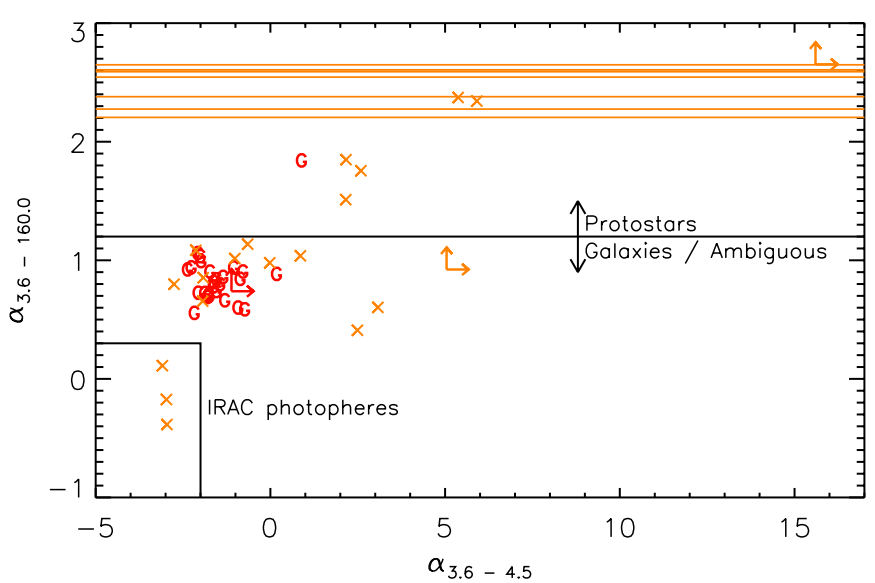

Figure 3. IRAC and PACS $160 \mu \mathrm{m}$ color indices $\alpha_{3.6-4.5}$ and $\alpha_{3.6-160}$ for the candidate protostar sample. Sources previously characterized as extragalactic based on their $\alpha_{5.8-8.0}>3$ index (or limit) are indicated in red with a "G." Sources with $\alpha_{5.8-8.0}<3$ are indicated in orange. The orange lines indicate the lower limits of $\alpha_{3.6-160}$ for sources with no IRAC detections. Sources with $\alpha_{3.6-160}>1.2$ are considered highly probable protostars, while other sources are flagged as described in the text.

(A color version of this figure is available in the online journal.)

with a very poorly constrained age of $\sim 0.06_{-0.03}^{+4.66} \mathrm{Myr}$. These authors only include data up to $24 \mu \mathrm{m}$. The SED we observe with Herschel may be consistent with a transition disk but not a debris disk. The remaining two sources in this category have similar SEDs as that of 069001; while a transition disk explanation for all three sources may appear likely depending on the age of the sources, we cannot currently rule out other possibilities. Nevertheless, all of these sources have SEDs consistent with a stellar photosphere in the IRAC bands, and hence these are likely to be fully formed stars surrounded by circumstellar dust.

Interestingly, we find that the most reliable SED classification criterion by far is that of sources that have neither IRAC nor MIPS $24 \mu \mathrm{m}$ detections. Of these, we find that all six sources have strong sub-millimeter detections and reside in dense and filamentary environments. This finding points to the critical importance of obtaining high-resolution sub-millimeter data to constrain the properties of such sources. In the following text, we include all $55 \mathrm{Herschel}$-detected sources in our analysis and figures.

\section{HERSCHEL PROTOSTAR CANDIDATES}

We present the Herschel protostar candidate catalog in Table 3. Here we include the PACS $70 \mu \mathrm{m}$ coordinates and flux measurements at $24 \mu \mathrm{m}, 70 \mu \mathrm{m}$, and $160 \mu \mathrm{m}$. We indicate which sources are flagged as reliable protostellar candidates and which are likely contamination, based on the results from the previous section. We also indicate if the sources have a robust $870 \mu \mathrm{m}$ detection. Furthermore, we present the values of $L_{\mathrm{bol}}$ and $T_{\text {bol }}$ and their corresponding estimated statistical errors (see discussion in Section 5.1). In Figure 5, we show the $70 \mu \mathrm{m}$ flux distributions for the sample compared to the distribution of HOPS protostars. The majority of the new candidate protostars have $70 \mu \mathrm{m}$ fluxes that are lower than the previously identified Spitzer HOPS sample; this is not surprising since the new candidate protostar sample is selected to be faint or undetected at $24 \mu \mathrm{m}$. Furthermore, the peak at low $70 \mu \mathrm{m}$ flux values is dominated by extragalactic contamination, as discussed above.

In Figure 6, we show the MIPS $24 \mu \mathrm{m}$, PACS $70 \mu \mathrm{m}$, and PACS $160 \mu \mathrm{m}$ colors of the new Herschel sources compared to 
Table 3

Herschel Protostar Candidate Coordinates and Photometry

\begin{tabular}{|c|c|c|c|c|c|c|c|c|c|c|c|}
\hline Source & $\begin{array}{l}\text { Group } \\
\text { Name }\end{array}$ & $\begin{array}{l}\text { R.A. }^{\mathrm{a}} \\
\text { (h:m:s) }\end{array}$ & $\begin{array}{l}\text { Decl. }^{\text {a }} \\
\left({ }^{\circ}:^{\prime \prime}\right)\end{array}$ & Field & $\begin{array}{c}24 \mu \mathrm{m} \\
(\mathrm{mJy})\end{array}$ & $\begin{array}{l}70 \mu \mathrm{m} \\
(\mathrm{mJy})\end{array}$ & $\begin{array}{c}160 \mu \mathrm{m} \\
(\mathrm{mJy})\end{array}$ & Flag $^{b}$ & $\begin{array}{c}870 \mu \mathrm{m} \\
\text { Detection }^{\mathrm{c}}\end{array}$ & $\begin{array}{l}T_{\text {bol }} \\
(\mathrm{K})\end{array}$ & $\begin{array}{l}L_{\text {bol }} \\
\left(L_{\odot}\right)\end{array}$ \\
\hline 061012 & 061 & $05: 42: 48.87$ & $-08: 16: 10.70$ & L1641 & $\leqslant 1.14$ & $703 \pm 35$ & $5634 \pm 845$ & 1 & $\cdots$ & $32.1 \pm 0.9$ & $0.75 \pm 0.06$ \\
\hline 119019 & 119 & 05:40:58.47 & $-08: 05: 36.10$ & L1641 & $1.46 \pm 0.2$ & $1604 \pm 80$ & $10745 \pm 1612$ & 1 & Yes & $34.4 \pm 0.8$ & $1.56 \pm 0.14$ \\
\hline 026011 & 026 & 05:39:17.00 & $-07: 24: 26.64$ & L1641 & $\leqslant 1.30$ & $78.4 \pm 3.9$ & $2516 \pm 377$ & 1 & Yes & $23.0 \pm 1.0$ & $0.34 \pm 0.03$ \\
\hline 313006 & 313 & 05:39:30.75 & $-07: 23: 59.40$ & L1641 & $\leqslant 1.25$ & $138 \pm 7$ & $2198 \pm 330$ & 1 & Yes & $27.8 \pm 1.0$ & $0.28 \pm 0.03$ \\
\hline 029003 & 029 & 05:39:13.15 & $-07: 13: 11.69$ & L1641 & $1.19 \pm 0.2$ & $29.5 \pm 1.5$ & $365 \pm 55$ & 1 & $\ldots$ & $42.0 \pm 1.5$ & $0.06 \pm 0.01$ \\
\hline 019003 & 019 & $05: 35: 23.92$ & $-05: 07: 53.46$ & ONC & $\leqslant 16.2$ & $2506 \pm 127$ & $31577 \pm 4737$ & 1 & Yes & $33.6 \pm 1.1$ & $3.16 \pm 0.31$ \\
\hline 082005 & 082 & $05: 41: 29.40$ & $-02: 21: 17.06$ & NGC 2024 & $\leqslant 3.13$ & $506 \pm 25$ & $9308 \pm 140$ & 1 & Yes & $29.3 \pm 0.8$ & $1.02 \pm 0.11$ \\
\hline 082012 & 082 & $05: 41: 24.94$ & $-02: 18: 08.54$ & NGC 2024 & $6.51 \pm 0.4$ & $4571 \pm 229$ & $51254 \pm 7688$ & 1 & Yes & $32.2 \pm 0.9$ & $6.27 \pm 0.65$ \\
\hline 090003 & 090 & $05: 42: 45.23$ & $-01: 16: 14.18$ & NGC 2024 & $4.74 \pm 0.3$ & $3286 \pm 164$ & $16937 \pm 2541$ & 1 & Yes & $36.0 \pm 0.8$ & $2.71 \pm 0.24$ \\
\hline 091015 & 091 & 05:46:07.65 & $-00: 12: 20.73$ & NGC 2068 & $\leqslant 1.29$ & $648 \pm 32$ & $6615 \pm 992$ & 1 & Yes & $30.9 \pm 0.8$ & $0.81 \pm 0.07$ \\
\hline 091016 & 091 & 05:46:09.97 & $-00: 12: 16.85$ & NGC 2068 & $\leqslant 1.14$ & $431 \pm 22$ & $5108 \pm 766$ & 1 & Yes & $29.1 \pm 0.9$ & $0.65 \pm 0.06$ \\
\hline 093005 & 093 & $05: 46: 27.75$ & $-00: 00: 53.81$ & NGC 2068 & $\leqslant 1.14$ & $1427 \pm 71$ & $12131 \pm 1820$ & 1 & Yes & $30.8 \pm 0.9$ & $1.71 \pm 0.15$ \\
\hline 302002 & 302 & $05: 46: 28.24$ & $00: 19: 27.00$ & NGC 2068 & $\leqslant 1.14$ & $302 \pm 15$ & $7187 \pm 108$ & 1 & Yes & $28.6 \pm 0.9$ & $0.84 \pm 0.09$ \\
\hline 097002 & 097 & 05:48:07.76 & $00: 33: 50.79$ & NGC 2068 & $\leqslant 1.14$ & $1049 \pm 52$ & $7993 \pm 120$ & 1 & Yes & $33.4 \pm 0.9$ & $1.14 \pm 0.11$ \\
\hline 300001 & 300 & 05:47:43.36 & $00: 38: 22.43$ & NGC 2068 & $\leqslant 7.19$ & $478 \pm 24$ & $5042 \pm 756$ & 1 & Yes & $29.6 \pm 0.9$ & $0.65 \pm 0.06$ \\
\hline 068006 & 068 & 05:41:11.79 & $-07: 53: 35.09$ & L1641 & $9.19 \pm 0.2$ & $44.8 \pm 2.2$ & $146 \pm 22$ & 2 & $\ldots$ & $149.7 \pm 5.06$ & $0.05 \pm 0.01$ \\
\hline 038002 & 038 & 05:36:11.11 & $-06: 49: 11.29$ & L1641 & $\leqslant 1.25$ & $36.4 \pm 1.8$ & $537 \pm 81$ & 2 & $\ldots$ & $40.4 \pm 1.0$ & $0.10 \pm 0.01$ \\
\hline 037003 & 037 & 05:37:00.35 & $-06: 37: 10.95$ & L1641 & $8.85 \pm 0.2$ & $725 \pm 36$ & $3798 \pm 570$ & 2 & $\ldots$ & $53.1 \pm 1.7$ & $0.43 \pm 0.03$ \\
\hline 037008 & 037 & $05: 37: 34.31$ & $-06: 35: 20.33$ & L1641 & $\leqslant 1.39$ & $27.7 \pm 1.4$ & $321 \pm 48$ & 2 & $\ldots$ & $45.5 \pm 1.9$ & $0.04 \pm 0.01$ \\
\hline 092011 & 092 & $05: 46: 26.17$ & $-00: 04: 45.31$ & NGC 2068 & $2.31 \pm 0.2$ & $77.8 \pm 3.9$ & $363 \pm 54$ & 2 & $\cdots$ & $104.1 \pm 5.63$ & $0.05 \pm 0.01$ \\
\hline 093001 & 093 & $05: 46: 56.32$ & $-00: 03: 14.73$ & NGC 2068 & $\leqslant 1.14$ & $21.8 \pm 1.1$ & $217 \pm 33$ & 2 & Yes & $47.9 \pm 0.7$ & $0.05 \pm 0.01$ \\
\hline 302004 & 302 & 05:46:16.55 & 00:21:35.09 & NGC 2068 & $2.87 \pm 0.2$ & $108 \pm 5$ & $283 \pm 42$ & 2 & $\ldots$ & $54.8 \pm 1.8$ & $0.07 \pm 0.01$ \\
\hline 096023 & 096 & $05: 46: 53.23$ & $00: 22: 10.05$ & NGC 2068 & $\leqslant 2.13$ & $147 \pm 7$ & $1691 \pm 254$ & 2 & $\ldots$ & $39.6 \pm 1.4$ & $0.18 \pm 0.02$ \\
\hline 301003 & 301 & $05: 46: 02.15$ & $00: 23: 29.86$ & NGC 2068 & $8.08 \pm 0.2$ & $42.6 \pm 2.1$ & $231 \pm 35$ & 2 & $\ldots$ & $92.1 \pm 3.8$ & $0.05 \pm 0.01$ \\
\hline 000011 & 000 & 05:54:32.10 & 01:42:54.92 & L1622 & $\leqslant 1.25$ & $28.5 \pm 1.4$ & $147 \pm 22$ & 2 & . & $50.1 \pm 0.5$ & $0.06 \pm 0.01$ \\
\hline 053002 & 053 & 05:43:24.07 & $-08: 49: 03.75$ & $\kappa$ Ori & $\leqslant 1.38$ & $33.5 \pm 1.7$ & $190 \pm 28$ & 3 & $\ldots$ & $43.2 \pm 0.3$ & $0.06 \pm 0.01$ \\
\hline 117004 & 117 & 05:41:40.40 & $-08: 41: 40.60$ & L1641 & $1.17 \pm 0.2$ & $25.3 \pm 1.3$ & $234 \pm 35$ & 3 & $\ldots$ & $56.4 \pm 2.2$ & $0.04 \pm 0.01$ \\
\hline 117014 & 117 & $05: 41: 29.28$ & $-08: 36: 14.60$ & L1641 & $1.20 \pm 0.2$ & $31.0 \pm 1.6$ & $237 \pm 36$ & 3 & $\ldots$ & $32.2 \pm 2.4$ & $0.11 \pm 0.01$ \\
\hline 119016 & 119 & $05: 40: 40.54$ & $-08: 05: 55.00$ & L1641 & $1.97 \pm 0.2$ & $42.7 \pm 2.1$ & $148 \pm 22$ & 3 & $\ldots$ & $62.7 \pm 1.7$ & $0.05 \pm 0.01$ \\
\hline 121011 & 121 & 05:41:37.90 & $-07: 55: 44.35$ & L1641 & $1.46 \pm 0.2$ & $74.1 \pm 3.7$ & $193 \pm 29$ & 3 & & $51.6 \pm 1.5$ & $0.04 \pm 0.01$ \\
\hline 025044 & 025 & 05:39:56.80 & $-07: 19: 21.40$ & L1641 & $\leqslant 1.18$ & $23.1 \pm 1.2$ & $150 \pm 22$ & 3 & $\ldots$ & $42.2 \pm 0.2$ & $0.08 \pm 0.01$ \\
\hline 030013 & 030 & 05:38:59.58 & $-07: 10: 31.92$ & L1641 & $1.43 \pm 0.2$ & $25.9 \pm 1.3$ & $153 \pm 23$ & 3 & & $48.0 \pm 1.0$ & $0.05 \pm 0.01$ \\
\hline 031003 & 031 & 05:38:55.35 & $-07: 05: 29.17$ & L1641 & $2.96 \pm 0.2$ & $49.9 \pm 2.5$ & $175 \pm 26$ & 3 & & $67.7 \pm 1.8$ & $0.06 \pm 0.01$ \\
\hline 034010 & 034 & $05: 37: 54.76$ & $-06: 56: 59.65$ & L1641 & $\leqslant 1.14$ & $61.3 \pm 3.1$ & $249 \pm 37$ & 3 & $\ldots$ & $43.5 \pm 0.7$ & $0.15 \pm 0.01$ \\
\hline 031037 & 031 & $05: 38: 28.20$ & $-06: 56: 40.16$ & L1641 & $\leqslant 1.14$ & $64.3 \pm 3.2$ & $396 \pm 59$ & 3 & $\ldots$ & $51.9 \pm 2.4$ & $0.06 \pm 0.01$ \\
\hline 034014 & 034 & 05:38:14.92 & $-06: 53: 03.56$ & L1641 & $1.89 \pm 0.2$ & $47.4 \pm 2.4$ & $235 \pm 35$ & 3 & & $65.5 \pm 3.5$ & $0.03 \pm 0.01$ \\
\hline 036003 & 036 & 05:38:05.97 & $-06: 50: 58.91$ & L1641 & $1.74 \pm 0.2$ & $67.4 \pm 3.4$ & $291 \pm 44$ & 3 & $\ldots$ & $74.6 \pm 3.1$ & $0.04 \pm 0.01$ \\
\hline 036006 & 036 & 05:37:45.07 & $-06: 50: 02.39$ & L1641 & $2.83 \pm 0.2$ & $34.8 \pm 1.7$ & $143 \pm 22$ & 3 & $\ldots$ & $60.1 \pm 1.8$ & $0.04 \pm 0.01$ \\
\hline 036011 & 036 & 05:37:42.72 & $-06: 47: 08.31$ & L1641 & $2.68 \pm 0.2$ & $41.8 \pm 2.1$ & $159 \pm 24$ & 3 & Yes & $65.9 \pm 1.9$ & $0.05 \pm 0.01$ \\
\hline 037011 & 037 & $05: 37: 37.80$ & $-06: 34: 43.35$ & L1641 & $2.42 \pm 0.2$ & $58.0 \pm 2.9$ & $409 \pm 61$ & 3 & $\ldots$ & $69.6 \pm 3.0$ & $0.04 \pm 0.01$ \\
\hline 050006 & 050 & 05:34:23.01 & $-06: 32: 58.00$ & L1641 & $2.82 \pm 0.2$ & $45.5 \pm 2.3$ & $313 \pm 47$ & 3 & $\ldots$ & $60.3 \pm 2.3$ & $0.06 \pm 0.01$ \\
\hline 037013 & 037 & 05:37:22.00 & $-06: 32: 56.48$ & L1641 & $2.93 \pm 0.2$ & $52.0 \pm 2.6$ & $180 \pm 27$ & 3 & $\ldots$ & $62.7 \pm 1.8$ & $0.05 \pm 0.01$ \\
\hline 041001 & 041 & $05: 36: 28.68$ & $-06: 30: 42.13$ & L1641 & $2.72 \pm 0.2$ & $47.5 \pm 2.4$ & $262 \pm 39$ & 3 & $\ldots$ & $90.7 \pm 4.8$ & $0.03 \pm 0.01$ \\
\hline 009001 & 009 & $05: 33: 32.55$ & $-05: 53: 34.25$ & ONC & $4.83 \pm 0.3$ & $74.9 \pm 3.8$ & $245 \pm 37$ & 3 & $\ldots$ & $95.4 \pm 2.6$ & $0.05 \pm 0.01$ \\
\hline 021010 & 021 & $05: 34: 19.63$ & $-04: 53: 23.54$ & ONC & $11.3 \pm 0.5$ & $316 \pm 16$ & $678 \pm 10$ & 3 & $\ldots$ & $63.8 \pm 2.2$ & $0.18 \pm 0.01$ \\
\hline 303017 & 303 & 05:47:49.01 & $00: 20: 26.47$ & NGC 2068 & $4.64 \pm 0.2$ & $107 \pm 5$ & $634 \pm 95$ & 3 & $\ldots$ & $70.2 \pm 3.8$ & $0.10 \pm 0.01$ \\
\hline 303023 & 303 & 05:47:45.58 & $00: 21: 14.68$ & NGC 2068 & $4.36 \pm 0.2$ & $65.9 \pm 3.3$ & $234 \pm 35$ & 3 & $\ldots$ & $104.1 \pm 4.81$ & $0.04 \pm 0.01$ \\
\hline 098001 & 098 & 05:47:25.77 & $00: 33: 37.43$ & NGC 2068 & $1.73 \pm 0.2$ & $9.79 \pm 0.5$ & $231 \pm 35$ & 3 & $\ldots$ & $43.5 \pm 2.1$ & $0.03 \pm 0.01$ \\
\hline 097003 & 097 & 05:47:45.88 & $00: 34: 12.71$ & NGC 2068 & $1.37 \pm 0.2$ & $13.0 \pm 0.7$ & $137 \pm 21$ & 3 & $\ldots$ & $78.1 \pm 4.4$ & $0.02 \pm 0.01$ \\
\hline 000003 & 000 & $05: 54: 17.28$ & 01:40:18.68 & L1622 & $8.63 \pm 0.4$ & $165 \pm 8$ & $386 \pm 58$ & 3 & $\ldots$ & $82.5 \pm 3.3$ & $0.10 \pm 0.01$ \\
\hline 000010 & 000 & 05:54:37.27 & 01:42:52.39 & L1622 & $5.10 \pm 0.3$ & $64.7 \pm 3.2$ & $215 \pm 32$ & 3 & $\ldots$ & $89.2 \pm 3.9$ & $0.04 \pm 0.01$ \\
\hline 069001 & 069 & $05: 40: 46.20$ & $-08: 04: 35.12$ & L1641 & $5.13 \pm 0.2$ & $325 \pm 16$ & $722 \pm 11$ & 4 & & $336.1 \pm 14.7$ & $0.29 \pm 0.01$ \\
\hline 026001 & 026 & 05:39:18.49 & $-07: 27: 52.37$ & L1641 & $4.71 \pm 0.2$ & $223 \pm 11$ & $598 \pm 90$ & 4 & $\ldots$ & $224.4 \pm 11.2$ & $0.17 \pm 0.01$ \\
\hline 306004 & 306 & 05:35:24.66 & $-04: 49: 43.53$ & ONC & $\leqslant 124$ & $435 \pm 22$ & $637 \pm 96$ & 4 & $\ldots$ & $119.1 \pm 3.56$ & $0.33 \pm 0.01$ \\
\hline 006006 & 006 & 05:35:11.47 & $-05: 57: 05.09$ & L1641 & $5.06 \pm 0.2$ & $126 \pm 6$ & $4027 \pm 604$ & 5 & Yes & $27.7 \pm 0.6$ & $0.31 \pm 0.04$ \\
\hline
\end{tabular}

Notes. Sources indicated in bold are those with $\log \left(\lambda F_{\lambda} 70 / \lambda F_{\lambda} 24\right)>1.65$. The ONC field contains the extended Orion Nebula region, the NGC 1977 region, and OMC2/3; the NGC 2024 field contains the NGC 2024 H II region and the NGC 2023 reflection nebula. The NGC 2068 field includes the NGC 2068 and NGC 2071 reflection nebulae as well as LBS23 region.

${ }^{a}$ PACS $70 \mu \mathrm{m}$ source coordinates.

${ }^{\mathrm{b}}$ This column indicates if a source is flagged as a reliable protostar (value $\left.=1\right)$, if the source is considered less likely to be a protostar $($ value $=2$ ), if the source is flagged as extragalactic contamination (value $=3$ ), and if the SED shape is with IRAC photospheric emission (value $=4)$. Finally, one source has no IRAC coverage $($ value $=5$ ). ${ }^{\mathrm{c}}$ This column indicates if the source has a strong $870 \mu \mathrm{m}$ detection; sources with no data are either assigned upper limits or have no coverage. 

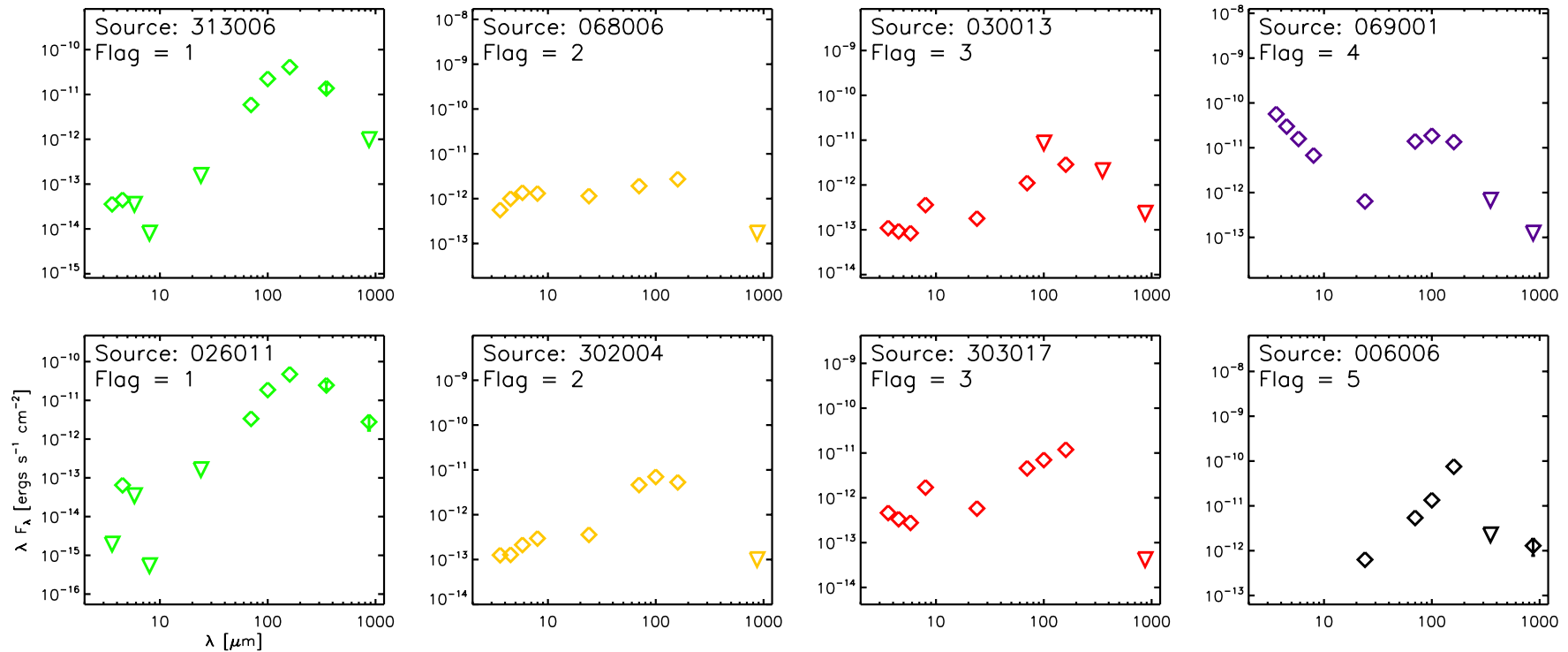

Figure 4. Example SEDs for each of the five categories described in Section 3.2: green SEDs (flag $=1$ ) are considered to be reliable protostar candidates, yellow SEDs $($ flag $=2)$ are less likely to be protostellar, red SEDs (flag $=3$ ) are considered extragalactic contamination based on the $8 \mu \mathrm{m}$ PAH feature, the purple SED (flag $=4$ ) is an example of one of the three sources that may be explained as transition disks, and, finally, the black SED (flag $=5$ ) is unclassifiable due to the lack of IRAC coverage. Errors are smaller than the size of the points, except for the $350 \mu \mathrm{m}$ and $870 \mu \mathrm{m}$ points; upper limits are indicated with triangles.

(A color version of this figure is available in the online journal.)

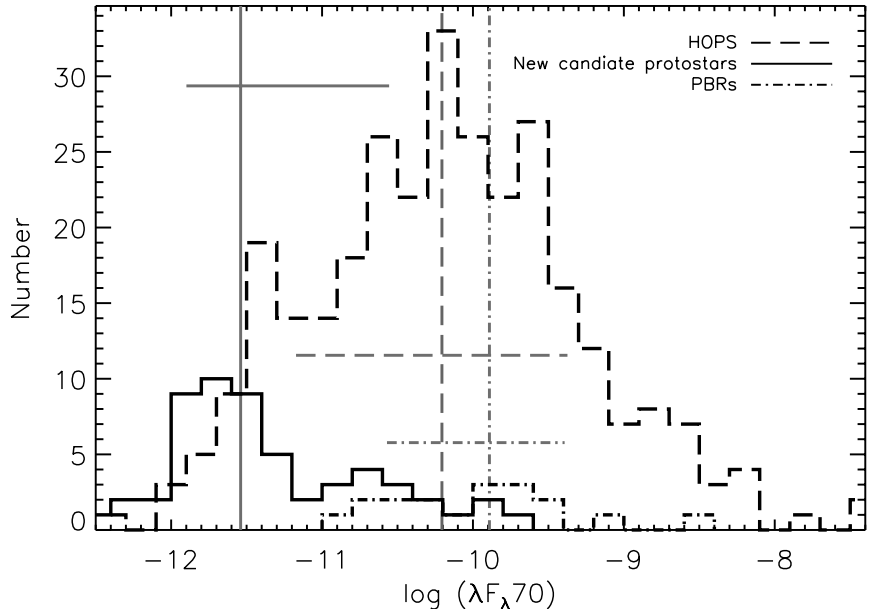

Figure 5. Distributions of $70 \mu \mathrm{m}$ fluxes for the HOPS protostars (dashed histogram), new candidate protostars (solid histogram), and the 18 reddest sources drawn from both combined samples (dot-dashed histogram). The corresponding gray lines indicate the median flux values (vertical lines) and the $68 \%$ interval (horizontal lines).

the HOPS sample of $70 \mu \mathrm{m}$ detected protostars. The top panel shows the $70 \mu \mathrm{m}$ flux versus the $\log \left(\lambda F_{\lambda} 70 / \lambda F_{\lambda} 24\right)$ color (henceforth $70 / 24$ color), while the bottom panel shows the $\log \left(\lambda F_{\lambda} 160 / \lambda F_{\lambda} 70\right)$ color (henceforth $160 / 70$ color) versus the $70 / 24$ color, for our sample of new protostar candidates compared to the colors of the Spitzer-identified HOPS sample. The Spitzer $24 \mu \mathrm{m} 7 \mathrm{mag}$ limit, imposed on the HOPS sample for a reliable protostellar identification, is apparent in the top panel as the diagonal line approximately separating the new protostellar candidates at fainter $70 \mu \mathrm{m}$ fluxes and redder $70 / 24$ colors from the population of Spitzer-identified HOPS sources.

For comparison, in the top panel of Figure 6, we also show the fluxes and colors of presumably typical and well-studied Class 0 sources: VLA1632-243 (J. Green and DIGIT team 2012, private communication and J. Green et al., in preparation), IRAS16293 (Evans et al. 2009), B335 (Stutz et al. 2008; Launhardt et al. 2013), CB68 (Launhardt et al. 2013), and CB244 (Stutz et al. 2010; Launhardt et al. 2013). Furthermore, we also show the colors of various VeLLOs: L673-7 (Dunham et al. 2008), IRAM04191 (Dunham et al. 2006), and CB130 (Launhardt et al. 2013). We find that the observed colors of our sample of candidate protostars appear consistent with the colors of more nearby Class 0 and VeLLO sources but not with FHSC candidate colors proposed in the literature (e.g., Commerçon et al. 2012). We find that the majority of these previously known Class 0 and VeLLO sources do not appear as red in their 70/24 colors as the reddest sources in our sample. The only exceptions to this trend are IRAS16293 and VLA1632-243, perhaps representing an extremum in the 70/24 color distribution that may be driven by their comparatively large envelope densities.

We also show in Figure 6 the colors of two FHSC candidates in Perseus: Per-Bolo 58 (Enoch et al. 2010) and B1-bS (Pezzuto et al. 2012). In this diagram, the 70/24 color of Per-Bolo 58 appears generally consistent with that of a VeLLO, as Enoch et al. (2010) point out. As such, this source may be an extremely low-mass protostar. On the other hand, the 70/24 color of $\mathrm{B} 1-\mathrm{bS}$ is comparable to the very reddest sources we find in Orion while the $70 \mu \mathrm{m}$ flux is consistent with VeLLOs and fainter than the reddest sources in Orion by more than one order of magnitude. The faint but robust detection of a $70 \mu \mathrm{m}$ point source by Pezzuto et al. (2012) may indeed point to the possible Class 0 or VeLLO nature of B1-bS. We do however note that Pezzuto et al. also detect a source with no $70 \mu \mathrm{m}$ counterpart, B1-bN, which may therefore represent a more robust FHSC candidate. Regardless of the elusive nature of FHSC candidates, when comparing our new candidate protostar colors to FHSC models by Commerçon et al. (2012), we find that our sources do not appear to be consistent with predicted or expected FHSC colors, with the caveat that distinguishing FHSCs from VeLLOs with continuum observations alone is likely difficult. 

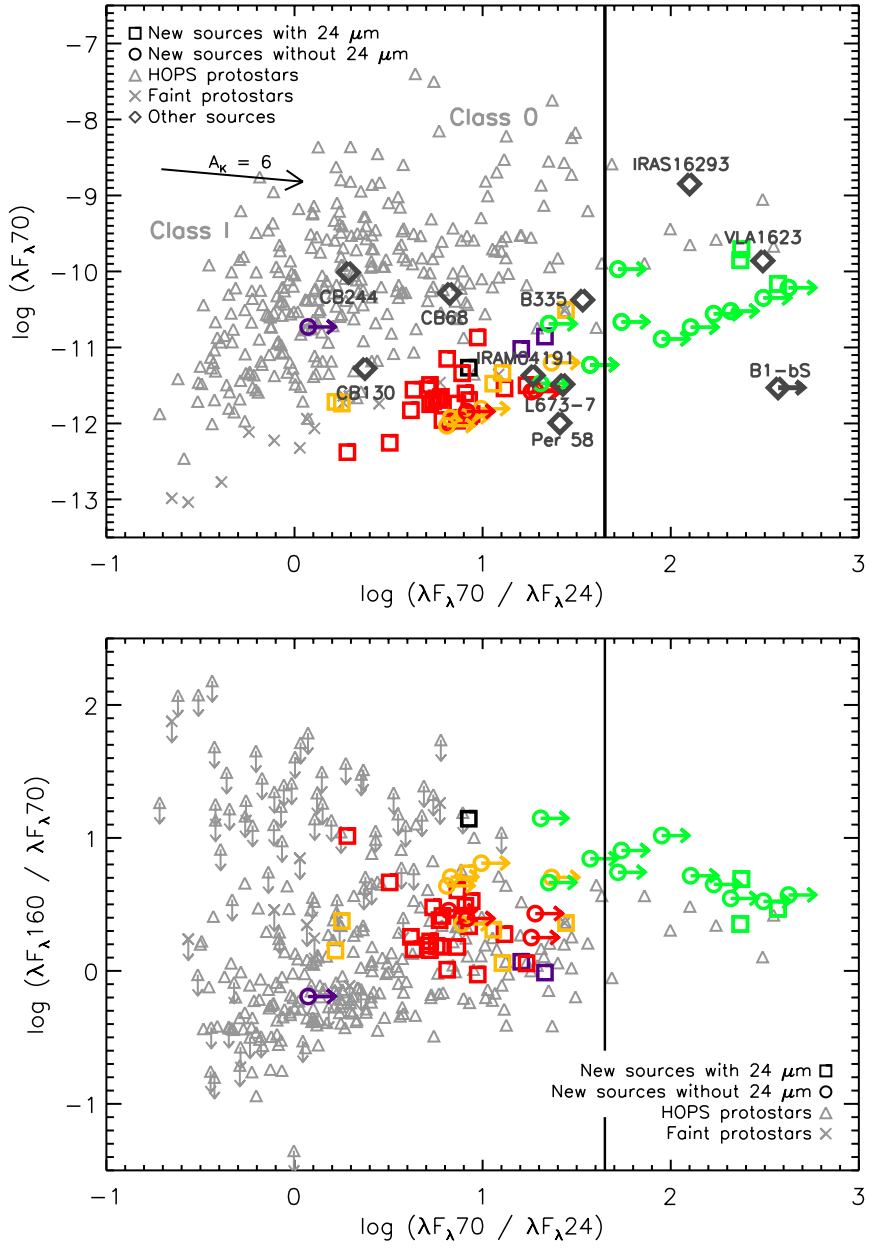

Figure 6. Top: $70 \mu \mathrm{m}$ flux vs. $70 \mu \mathrm{m}$ to $24 \mu \mathrm{m}$ flux ratio for HOPSdetected Orion protostars. Open squares and open circles indicate the new candidate protostars with and without MIPS $24 \mu \mathrm{m}$ detections, respectively. The color of the symbols indicates the flag values shown in Table 3 and discussed in Section 3.2: here, green indicates sources that are reliable protostar candidates $($ flag $=1)$, orange indicates less reliable sources $($ flag $=2)$, red indicates extragalactic contamination $($ flag $=3$ ), purple indicates other sources $($ flag $=4)$, and black indicates the single source without IRAC coverage. The solid vertical line indicates a $70 \mu \mathrm{m}$ to $24 \mu \mathrm{m}$ flux ratio of 1.65 , our PBRs selection criterion (see Section 5). Triangles and $\times$ symbols indicate previously detected and characterized Spitzer protostars and faint protostellar candidates from Megeath et al. (2012), respectively. The arrow indicates the extinction vector for a value of $A_{K}=6$. For comparison, we also indicate as large diamonds the scaled observed measurements for some well-known Class 0 sources, VeLLOs, and two FHSC candidates (Per 58 and B1-bS; see Section 4). Bottom: $160 \mu \mathrm{m}$ to $70 \mu \mathrm{m}$ flux ratio vs. $70 \mu \mathrm{m}$ to $24 \mu \mathrm{m}$ flux ratio for HOPSdetected Orion protostars. The symbol and color type is the same as in the top panel. The reddest sources are distinguished from the bulk of Spitzer-identified HOPS sources (triangles) both by brighter $160 \mu \mathrm{m}$ fluxes and redder $70 \mu \mathrm{m}$ to $24 \mu \mathrm{m}$ flux ratios.

(A color version of this figure is available in the online journal.)

\section{PACS BRIGHT RED SOURCES}

Up to this point, we have discussed two distinct and welldefined samples of sources in Orion: (1) the sample of candidate protostars identified with Herschel that have PACS $70 \mu \mathrm{m}$ and $160 \mu \mathrm{m}$ detections but MIPS $24 \mu \mathrm{m}$ magnitudes greater than $7.0 \mathrm{mag}$ and (2) the sample of protostars that were reliably identified with Spitzer (24 $\mu$ m magnitudes $\leqslant 7.0$ mag; Megeath et al. 2012; Kryukova et al. 2012). The protostar catalog target list used for the HOPS program consists mostly of the Spitzer identified protostars, but also contains some of the previously known protostars with $m(24)>7.0 \mathrm{mag}$ (W. J. Fischer et al., in preparation).

In what follows, we focus our analysis on the Orion protostars that have $70 / 24>1.65$. Of the 18 known protostars that satisfy this limit, 11 are identified with Herschel; hence this color regime is dominated by our newly identified protostars. Accordingly, Herschel has provided us for the first time with a far more complete sample of these red sources within the field of the HOPS survey. Given their red colors and their brightness in the PACS wavelength bands, we refer to this sample of protostars as PBRs. The coordinates, Spitzer photometry, and Herschel photometry of the sample are listed in Table 4, while the APEX $350 \mu \mathrm{m}$ and $870 \mu \mathrm{m}$ photometry are presented in Table 5. Since the APEX photometry are non-trivial to extract due in large part to contamination by cold surrounding material and, at $870 \mu \mathrm{m}$ specifically, by the large beam size, we present three measures of the source flux, as described above.

\subsection{Observed Properties of PBRs}

As discussed above, we select 18 PBRs in Orion with observed 70/24 colors greater than 1.65 . We show $4.5 \mu \mathrm{m}$ to $870 \mu \mathrm{m}$ images of five example PBRs in Figures 7 and 8 (see Appendix B for the full sample images). Furthermore, in Figure 9 we show the full set of 18 PBRs' observed SEDs from $24 \mu \mathrm{m}$ to $870 \mu \mathrm{m}$. Inspection of the observed SEDs confirms that the PBRs sample is composed of cold, envelope-dominated sources with peak emission always located at $\lambda>70 \mu \mathrm{m}$. In addition, the peak of the SEDs, and thus the temperatures, are well constrained for all PBRs because we have obtained APEX sub-millimeter coverage for all sources.

In Table 6, we present some basic properties of the PBRs. In particular, we find that $12 / 18$ sources exhibit Spitzer $4.5 \mu \mathrm{m}$ emission indicative of outflow activity. We also include some references to previous detections (see Appendix A). Furthermore, $4 / 18$ sources have significant levels of $4.5 \mu \mathrm{m}$ emission that are indeed consistent with a high inclination. The majority of sources, however, do not give clear indications of their inclinations at any observed wavelength, and therefore we cannot make any statements about their possible orientations based on their appearance in the images. We find indications from the $4.5 \mu \mathrm{m}$ image morphology that two sources (HOPS341 and HOPS354) are binaries, while seven sources have a nearby source within $30^{\prime \prime}$. Two reside in more crowded regions, and seven sources appear truly isolated. We find that a significant fraction (13/ 18) of sources appear to reside in filamentary regions, i.e., the extended $870 \mu \mathrm{m}$ emission appears significantly elongated.

The four sources with significant indications of a high inclination orientation are HOPS169, 302002, HOPS341, and HOPS354 (see Appendix B for Figures 17, 21, 23, and 25). Inspection of their $4.5 \mu \mathrm{m}$ images reveals that their outflows appear well collimated and relatively narrow. Indeed, we might expect that sources that have denser envelopes, and are therefore presumably younger, may have narrower cavity opening angles (e.g., Arce \& Sargent 2006). As an additional check on our density analysis (see Section 6), we use this inclination information for an independent check of the envelope densities of these four sources. Despite the relatively sparsely sampled SEDs, we fix the inclination to $87^{\circ}$ and fit the source SEDs. We find that even when we fix the model inclination to $\theta=87^{\circ}$, we still obtain envelope densities significantly above the $\rho_{1}$ value found in Section 6. 
Table 4

Spitzer and Herschel Photometric Properties of PACS Bright Red Sources

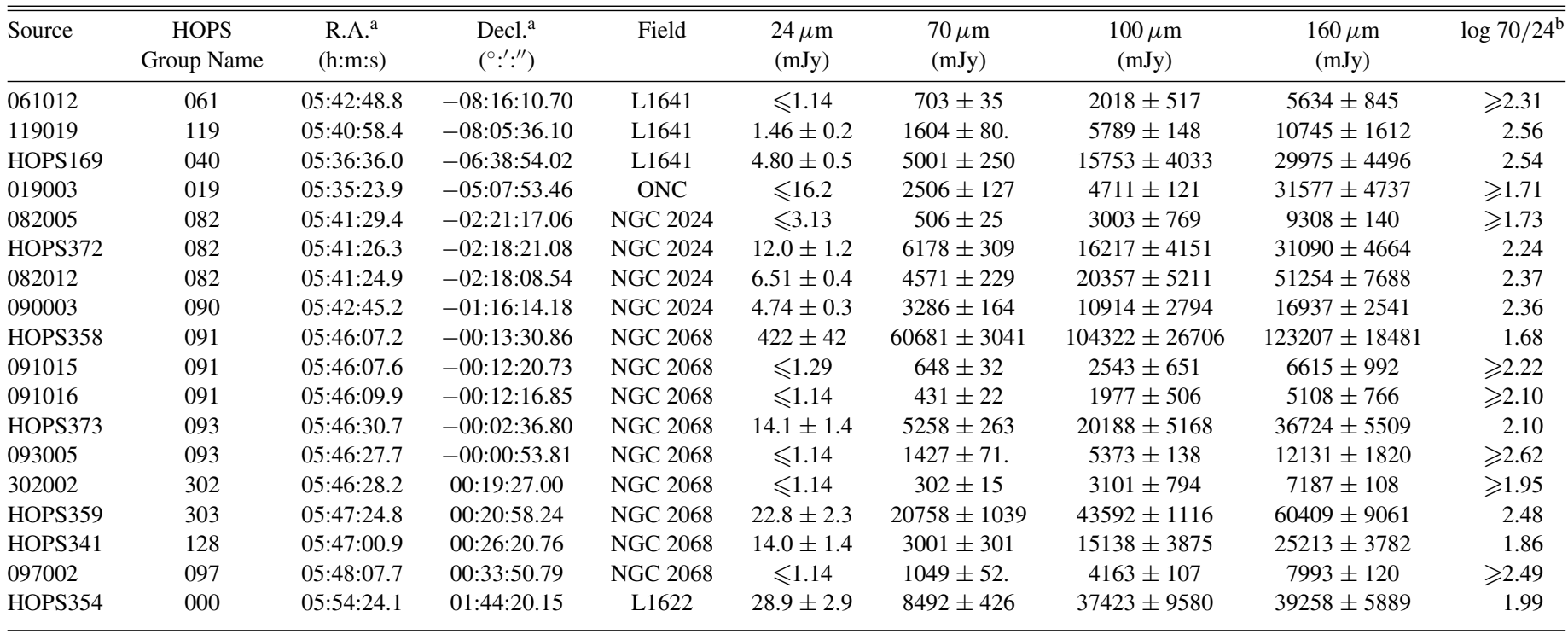

Notes. The ONC field contains the extended Orion Nebula region, the NGC 1977 region, and OMC2/3; the NGC 2024 field contains the NGC 2024 H II region and the NGC 2023 reflection nebula. The NGC 2068 field includes the NGC 2068 and NGC 2071 reflection nebulae as well as LBS23 region.

a Object coordinates are derived from the PACS $70 \mu \mathrm{m}$ images.

${ }^{\mathrm{b}} \log \lambda F_{\lambda} 70 / \lambda F_{\lambda} 24$.

Table 5

APEX 350 and $870 \mu \mathrm{m}$ Photometry of PACS Bright Red Sources

\begin{tabular}{lccccrr}
\hline \hline Source & $\begin{array}{c}350 \mu \mathrm{m} \\
\left(\mathrm{Jy} \mathrm{beam}^{-1}\right)\end{array}$ & $\begin{array}{c}350 \mu \mathrm{m}^{\mathrm{a}} \\
(\mathrm{Jy})\end{array}$ & $\begin{array}{c}350 \mu \mathrm{m}^{\mathrm{b}} \\
(\mathrm{Jy})\end{array}$ & $\begin{array}{c}870 \mu \mathrm{m} \\
\left(\mathrm{Jy} \mathrm{beam}^{-1}\right)\end{array}$ & $\begin{array}{c}870 \mu \mathrm{m}^{\mathrm{a}} \\
(\mathrm{Jy})\end{array}$ & $\begin{array}{c}870 \mu \mathrm{m}^{\mathrm{b}} \\
(\mathrm{Jy})\end{array}$ \\
\hline 061012 & 2.60 & 3.93 & 2.53 & $\leqslant 0.7$ & $\leqslant 1.3$ & $\leqslant 1.3$ \\
119019 & 3.38 & 5.27 & 3.63 & 0.5 & 0.9 & 0.6 \\
HOPS169 & $\ldots$ & $\ldots$ & $\ldots$ & 1.0 & 1.6 & 1.4 \\
019003 & 8.29 & 19.6 & 7.18 & 2.5 & 5.0 & 3.0 \\
082005 & $\ldots$ & $\ldots$ & $\ldots$ & 0.8 & 1.6 & 1.0 \\
HOPS372 & $\ldots$ & $\ldots$ & $\ldots$ & $\leqslant 1.4$ & $\leqslant 4.0$ & $\leqslant 4.0$ \\
082012 & $\ldots$ & $\ldots$ & $\ldots$ & 2.7 & 4.3 & 3.6 \\
090003 & $3.63^{\mathrm{c}}$ & $\ldots$ & $\ldots$ & 1.7 & 2.2 & 1.9 \\
HOPS358 & 13.0 & 22.4 & 17.3 & 1.8 & 3.3 & 2.6 \\
091015 & 2.14 & 3.15 & 2.26 & 0.6 & 1.3 & 0.5 \\
091016 & 2.50 & 3.76 & 2.81 & 0.7 & 1.2 & 0.8 \\
HOPS373 & 9.02 & 12.9 & 10.5 & 1.5 & 2.5 & 2.0 \\
093005 & 6.58 & 10.1 & 6.74 & 1.4 & 2.6 & 1.6 \\
302002 & $\ldots$ & $\ldots$ & $\ldots$ & 1.1 & 1.7 & 1.5 \\
HOPS359 & $\ldots$ & $\ldots$ & $\ldots$ & 1.8 & 2.9 & 2.2 \\
HOPS341 & $\ldots$ & $\ldots$ & $\ldots$ & 1.0 & 1.5 & 1.3 \\
097002 & $\ldots$ & $\ldots$ & $\ldots$ & 0.6 & 0.9 & 0.8 \\
HOPS354 & 13.8 & 25.9 & 18.7 & 1.5 & 2.7 & 2.2 \\
\hline
\end{tabular}

Notes.

a Source flux measured in an aperture with radius equal to $1 \times$ FWHM, where the FWHM $=7$ ". 34 and 19 '. 0 at 350 and $870 \mu \mathrm{m}$, respectively.

${ }^{\mathrm{b}}$ Source flux measured in the same aperture as in "a" but with local sky subtraction over radii equal to $\{1.5,2.0\} \times$ FWHM.

c $350 \mu \mathrm{m}$ point from Miettinen et al. (2012).

\subsection{Observational Evolutionary Diagnostics}

We calculate $L_{\mathrm{bol}}, T_{\mathrm{bol}}$ (Myers \& Ladd 1993), and $L_{\mathrm{smm}} / L_{\mathrm{bol}}$ (André et al. 1993, 2000). The errors in $L_{\mathrm{bol}}$ and $T_{\mathrm{bol}}$ are derived with the same Monte Carlo method as described in Section 6.2 for the modified blackbody parameters. We exclude the IRAC upper limits from this analysis; including these limits has an effect on our $L_{\mathrm{bol}}$ and $T_{\mathrm{bol}}$ estimates that is smaller than our estimated errors. We do, however, include the $24 \mu \mathrm{m}$ upper limits; therefore, the $L_{\mathrm{bol}}$ and $T_{\mathrm{bol}}$ values should be considered upper limits for sources not detected at this wavelength. Furthermore, we investigate the effect of applying an average foreground reddening correction to all the new Herschel candidate protostars. We find that dereddening the observed fluxes with extinction levels of $A_{V}=40 \mathrm{mag}$ has no effect on the derived parameters because the observed SEDs are extremely red and cold. 

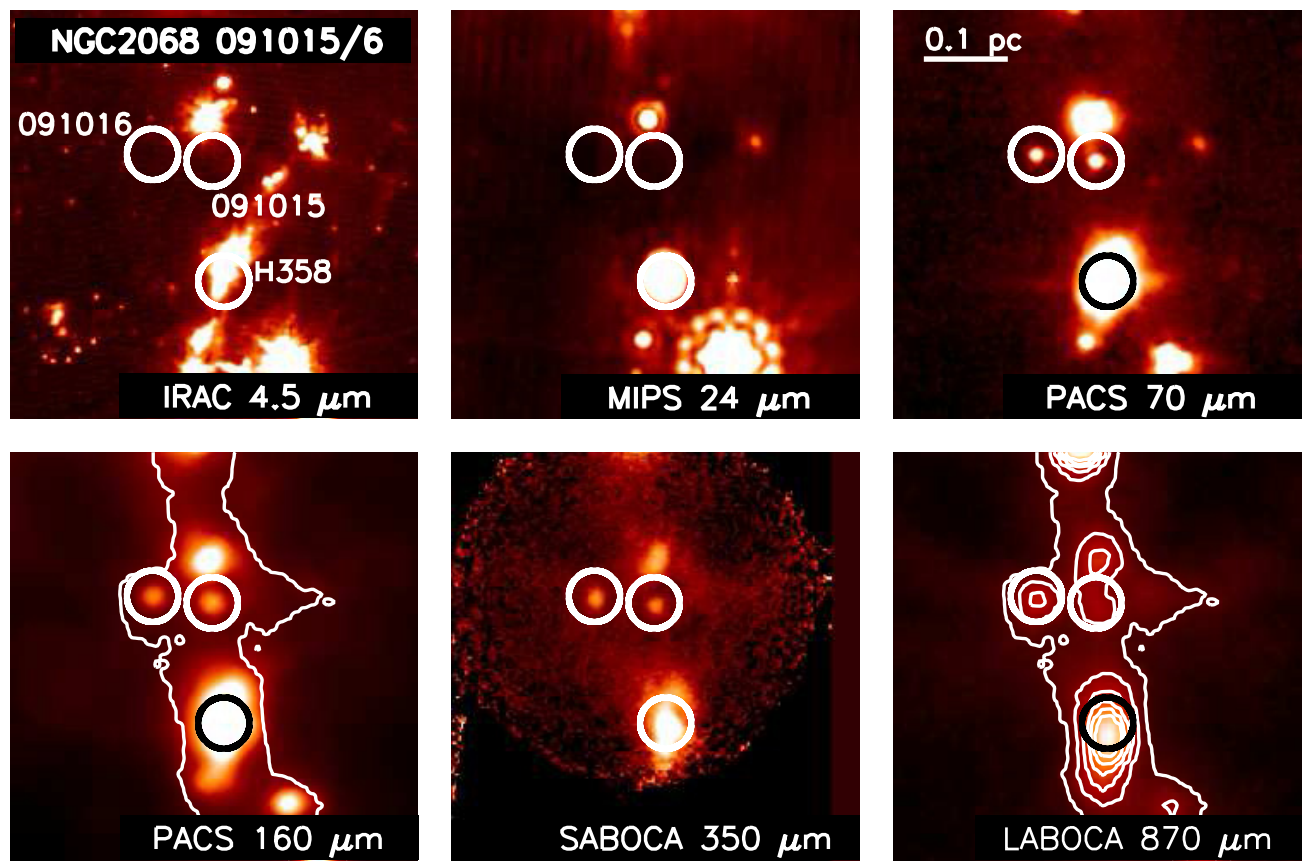

Figure 7. $4^{\prime} \times 4^{\prime}$ images of three PBR sources, 091015 and 091016 (top), and HOPS358 (bottom), at the indicated wavelengths, shown on a log scale. North is up and east is to the left. The circles indicate the location of the PACS $70 \mu \mathrm{m}$ point sources. Contours indicate the $870 \mu \mathrm{m}$ emission levels at $\{0.25,0.5,0.75,1.0,1.25\}$ $\mathrm{Jy} \mathrm{beam}^{-1}$. The lowest $870 \mu \mathrm{m}$ contour is overplotted on the $160 \mu \mathrm{m}$ image. No IRAC emission coincident with 091015 or 091016 is detected; however, these sources clearly reside in dense filamentary material traced by the sub-millimeter emission. The HOPS358 photometry is likely blended.

(A color version of this figure is available in the online journal.)
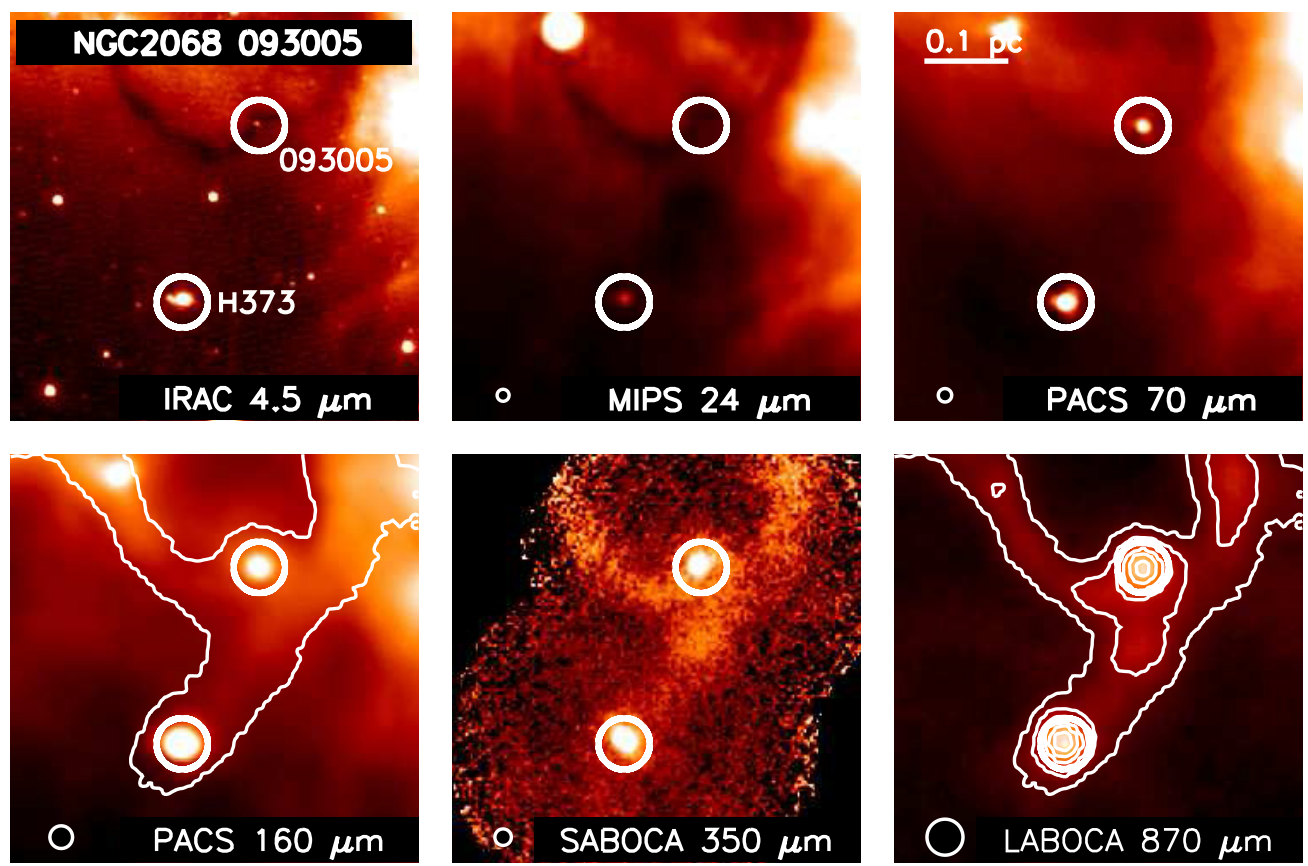

Figure 8. Same as Figure 7, showing $4^{\prime} \times 4^{\prime}$ images of two PBRs: 093005 (top) and HOPS373 (bottom). Contours indicate the $870 \mu \mathrm{m}$ emission levels at $\{0.25$, $0.5,0.75,1.0,1.25,1.5\} \mathrm{Jy} \mathrm{beam}^{-1}$. Source 093005 is the reddest PBR shown in Figure 6 and lies at the intersection of three filaments traced by an 8 and $24 \mu \mathrm{m}$ absorption feature and the $870 \mu \mathrm{m}$ emission.

(A color version of this figure is available in the online journal.)

\subsection{Spatial Distribution of the Three Samples}

We show the locations of the Herschel protostar candidates compared to the locations of the HOPS sample in Figure 10; these positions are overlaid on the extinction map of Orion. It is immediately apparent that the spatial distribution of the new candidate protostars and PBRs is non-uniform. To investigate this distribution further, we show the relative fraction of new sources as a function of individual region in Table 7. The overall number of new candidate protostars and PBRs is dominated by the Orion A cloud, and in particular L1641. This is not surprising since the L1641 region is quite large and contains 

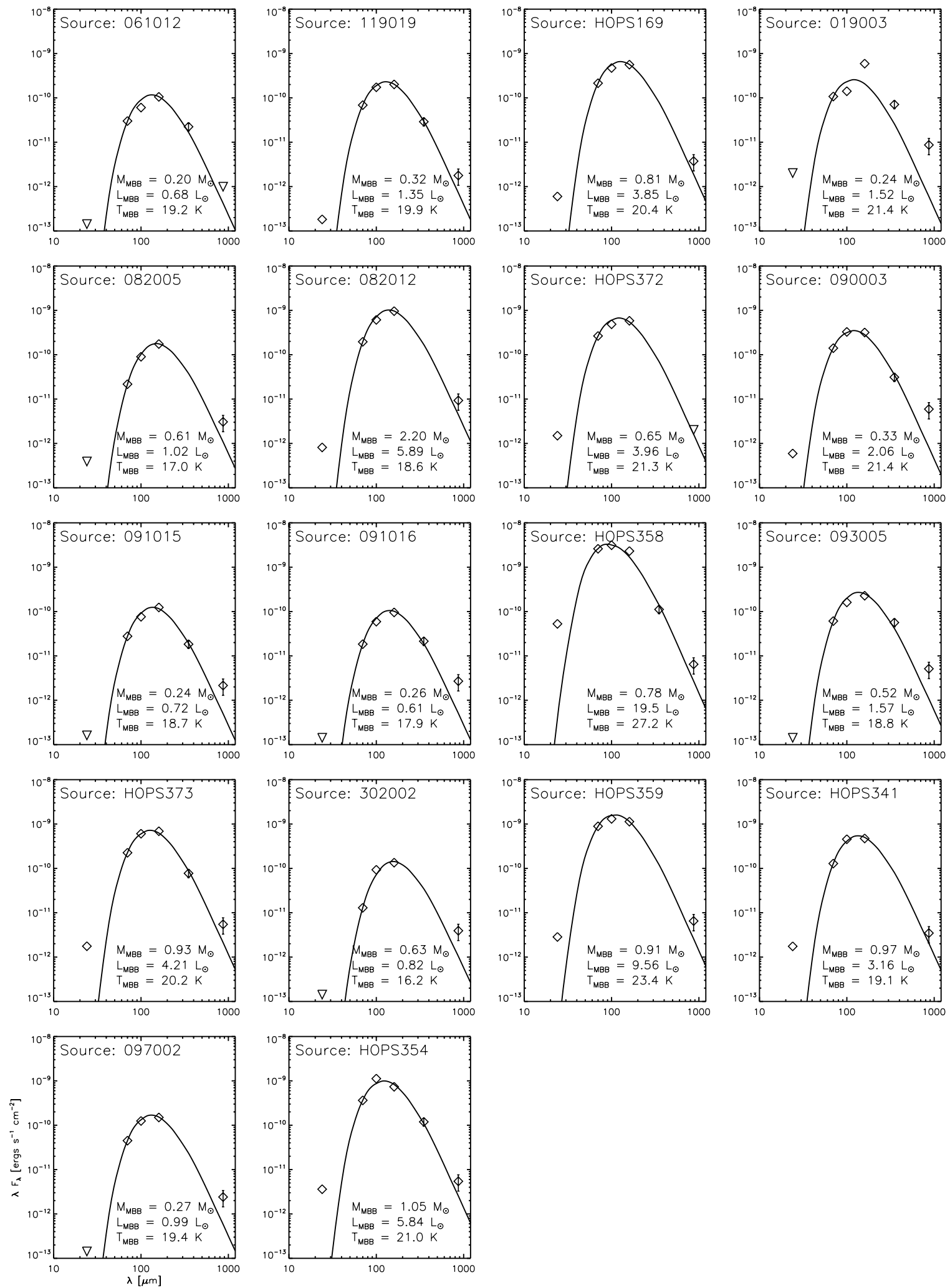

Figure 9. SEDs of the 18 PBRs are shown. The errors are smaller than the symbol size except for the 350 and $870 \mu \mathrm{m}$ data points. The black curve shows the modified blackbody fit to the observed SED, with the indicated best-fit parameters (see Section 6.2). Note that PBR 019003 (shown in the top right) is located in a complex field where the photometry may be strongly contaminated.

more protostars compared to other Orion regions. The fractions of new candidate protostars and PBRs compared to the total number of HOPS and new candidate protostars is, however, two times larger in Orion B. This result is even more pronounced when we consider only the fractions of PBRs, with fractions that are more than 10 times larger in Orion B. The NGC 2068 (also containing the NGC 2071 nebula) and NGC 2024 (also containing the Horsehead or NGC 2023 nebula) fields in Orion B not only have the largest fraction of new candidate protostars, but also of PBRs. While these numbers and fractions are subject 
Table 6

Orion PBRs Observed Properties: Detections, Environment, and Previous Detections

\begin{tabular}{|c|c|c|c|c|c|c|c|}
\hline Source & $\begin{array}{c}\text { R.A. } \\
\text { (h:m:s) }\end{array}$ & $\begin{array}{l}\text { Decl. } \\
\left({ }^{\circ}:^{\prime \prime}:^{\prime}\right)\end{array}$ & $\begin{array}{c}4.5 \mu \mathrm{m} \\
\text { Detection }\end{array}$ & $\begin{array}{l}\text { High } \\
\text { Incl. }^{\mathrm{a}}\end{array}$ & $\begin{array}{c}24 \mu \mathrm{m} \\
\text { Detection }\end{array}$ & Note $^{b}$ & $\begin{array}{c}\text { Selected } \\
\text { References }\end{array}$ \\
\hline 061012 & $05: 42: 48.8$ & $-08: 16: 10.7$ & Yes & $\ldots$ & No & ns & $\ldots$ \\
\hline 119019 & 05:40:58.4 & $-08: 05: 36.1$ & Yes & $\ldots$ & Yes & $\mathrm{f}, \mathrm{i}$ & $\ldots$ \\
\hline HOPS169 & $05: 36: 36.0$ & $-06: 38: 54.0$ & Yes & Yes & Yes & $\mathrm{f}, \mathrm{i}$ & Le88, Mo91, Za97, Da00, St02, Jo06, Nu07, Ba09, Me12 \\
\hline 019003 & $05: 35: 24.0$ & $-05: 07: 50.1$ & Yes & $\ldots$ & No & $\mathrm{f}, \mathrm{c}$ & Me90, Ch97, Ni03, Ts03, Nu07, Me12 \\
\hline 082005 & $05: 41: 29.4$ & $-02: 21: 17.1$ & No & $\ldots$ & No & $\mathrm{f}, \mathrm{i}$ & La91, La96, Mo99, Jo06, Nu07 \\
\hline HOPS372 & $05: 41: 26.3$ & $-02: 18: 21.1$ & Yes & $\ldots$ & Yes & $\mathrm{f}, \mathrm{ns}$ & La91, La96, Mo99, Jo06, Nu07, Me12 \\
\hline 082012 & $05: 41: 24.9$ & $-02: 18: 08.5$ & No & $\ldots$ & Yes & f,ns & La91, La96, Mo99, Wu04, Jo06, Nu07 \\
\hline 090003 & $05: 42: 45.2$ & $-01: 16: 14.2$ & Yes & $\ldots$ & Yes & $\mathrm{i}$ & Mi09 \\
\hline HOPS358 & $05: 46: 07.2$ & $-00: 13: 30.9$ & Yes & $\ldots$ & Yes & $\mathrm{f}, \mathrm{c}$ & St86, Li99, Re99, Mi01, Wu04, Nu07, Me12 \\
\hline 091015 & 05:46:07.7 & $-00: 12: 19.1$ & No & $\ldots$ & No & f,ns & Li99, Mi01, Nu07 \\
\hline 091016 & $05: 46: 10.0$ & $-00: 12: 15.4$ & No & $\ldots$ & No & f,ns & Li99, Mi01, Nu07 \\
\hline HOPS373 & $05: 46: 30.7$ & $-00: 02: 36.8$ & Yes & $\ldots$ & Yes & $\mathrm{f}, \mathrm{i}$ & Ha83, La91, Gi00, Mo01, Mi01, Nu07, Me12 \\
\hline 093005 & $05: 46: 27.7$ & $-00: 00: 51.5$ & Yes & $\ldots$ & No & $\mathrm{f}, \mathrm{i}$ & La91, Mo01, Mi01, Nu07 \\
\hline 302002 & $05: 46: 28.2$ & $+00: 19: 28.4$ & Yes & Yes & No & $\mathrm{f}, \mathrm{ns}$ & La91, Jo01, Ph01, Mo01, Sa03, Nu07 \\
\hline HOPS359 & $05: 47: 24.8$ & $+00: 20: 58.2$ & No & $\ldots$ & Yes & $\mathrm{f}, \mathrm{i}$ & La91, Mo01, Nu07, Me12 \\
\hline HOPS341 & $05: 47: 00.9$ & $+00: 26: 20.8$ & Yes & Yes & Yes & $\mathrm{b}$ & Jo01, Mo01, Nu07, Sa10, Me12 \\
\hline 097002 & 05:48:07.7 & $+00: 33: 52.7$ & No & $\ldots$ & No & $\mathrm{b} / \mathrm{ns}$ & $\ldots$ \\
\hline HOPS354 & $05: 54: 24.2$ & $+01: 44: 20.1$ & Yes & Yes & Yes & $\mathrm{b}$ & $\operatorname{Re} 08, \mathrm{Ba} 09, \mathrm{Me} 12$ \\
\hline
\end{tabular}

\section{Notes.}

${ }^{\text {a }}$ Sources with indications of an observed high inclination orientation from the $4.5 \mu \mathrm{m}$ images.

${ }^{\mathrm{b}} \mathrm{ns}=$ nearby source and indicates that we find a source close $\left(\lesssim 30^{\prime \prime}\right)$ to the target; $\mathrm{f}=$ filament and indicates that a significant level of elongation or extended emission is seen in the $870 \mu \mathrm{m}$ data; $\mathrm{i}=$ isolated and indicates that no nearby source is observed; $\mathrm{c}=$ crowded, indicating that the source resides in a region possibly with multiple sources, extended emission, or both and whose photometry may be affected by source blending; finally, $\mathrm{b}=$ binary, sources that may have an unresolved secondary source and therefore blended photometry.

References. (Le88) Levreault 1988; (Mo91) Morgan et al. 1991; (ZA97) Zavagno et al. 1997; (Da00) Davis et al. 2000; (St02) Stanke et al. 2002; (Da02) Davis et al. 2009; (Nu07) Nutter \& Ward-Thompson 2007, Di Francesco et al. 2008; (Jo06) Johnstone \& Bally 2006; (Ts03) Tsujimoto et al. 2003; (Ch97) Chini et al. 1997; (Ni03) Nielbock et al. 2003; (Me90) Mezger et al. 1990; (Mo99) Mookerjea et al. 2009; (La96) Launhardt et al. 1996; (La91) Lada et al. 1991a; (Wu04) Wu et al. 2004; (Mi09) Miettinen et al. 2009; (Re99) Reipurth et al. 1999; (St86) Strom et al. 1986; (Li99) Lis et al. 1999; (Mi01) Mitchell et al. 2001; (Mo01) Motte et al. 2001; (Gi00) Gibb \& Little 2000; (Ha83) Haschick et al. 1983; (Ph01) Phillips et al. 2001; (Sa03) Savva et al. 2003; (Jo01) Johnstone et al. 2001; (Re08) Reipurth et al. 2008; (Ba09) Bally et al. 2009; (Sa10) Sadavoy et al. 2010; (Me12) Megeath et al. 2012.

Table 7

Numbers and Fractions of New Sources and Red PBRs Found by Region

\begin{tabular}{lcccccc}
\hline \hline Region & Field & $\begin{array}{c}\text { Total } \\
\text { Number }\end{array}$ & $\begin{array}{c}\text { New } \\
\text { Number }\end{array}$ & $\begin{array}{c}\text { New } \\
\%\end{array}$ & $\begin{array}{c}\text { PBRs } \\
\text { Number }\end{array}$ & $\begin{array}{c}\text { PBRs } \\
\%\end{array}$ \\
\hline All & & 355 & 56 & $16 \%$ & 18 & $5 \%$ \\
Orion A & & 274 & 35 & $13 \%$ & 4 & $1 \%$ \\
& L1641 $^{\text {a }}$ & 200 & 31 & $16 \%$ & 3 & $2 \%$ \\
& ONC & 74 & 4 & $5 \%$ & 1 & $1 \%$ \\
Orion B & & 81 & 21 & $26 \%$ & 14 & $17 \%$ \\
& NGC 2024 & 17 & 3 & $18 \%$ & 4 & $24 \%$ \\
& NGC 2068 & 52 & 15 & $29 \%$ & 9 & $17 \%$ \\
& L1622 & 12 & 3 & $25 \%$ & 1 & $8 \%$ \\
\hline
\end{tabular}

Note. ${ }^{a}{ }^{\kappa}$ Ori sources have been included here.

to counting statistics and other possibly large sources of errors, the differences between Orion A and Orion B appear to be significant.

About $5 \%$ of the combined protostars and candidate protostars in Orion are PBRs. If we consider the PBRs as representing a distinct phase in the evolution of a protostar, and we assume a constant rate of star formation, this fraction suggests that the protostars spend 5\% of their lifetime in the PBRs phase (approximately 25,000 years with the $0.5 \mathrm{Myr}$ protostellar lifetime of Evans et al. 2009), averaging over all Orion regions. However, due to the spatial variations in the fractions of PBRs, the resulting duration of the PBR phase would vary greatly with location, from 5000 years in the Orion A cloud to 80,000 years in the Orion B cloud. There are two alternative explanations for the large variations in percentage of PBRs. First, there might be environmental reasons which would favor the formations of PBRs, or perhaps extend the duration of the PBRs phase, in the Orion B cloud. Second, the ages of all the protostars in the Orion B cloud may be systematically younger than those in the Orion A cloud. In this case, the regions containing the PBRs in the Orion B could be undergoing very recent bursts of star formation.

Studies of pre-main-sequence stars in the Orion molecular clouds show little evidence for significant age differences between the Orion A and B clouds. Flaherty \& Muzerolle (2008) determined an age of 2 Myr for NGC 2068 and NGC 2071, while Reggiani et al. (2011), Hsu et al. (2012), and Da Rio et al. (2012) determine ages for the ONC and L1641 of 2-3 Myr. However, most of the protostars associated with the NGC 2068 and NGC 2071 regions are outside the clusters of pre-mainsequence stars and in dense filaments and gas neighboring these clusters (Motte et al. 2001). A number of PBRs are in the LBS23 clump (directly south of NGC 2068) and in the NGC 2023 clump (in the NGC 2024 field); these are two of the five most massive, dense clumps found in Orion (Lada et al. 1991a). Compared to the other massive clumps, both of these regions have $1 / 10$ the numbers of young stars per unit gas mass and hence may be quite young (Lada et al. 1991b; Lada 1992). The southern rim of the NGC 2068 nebula, LBS23, and the northern rim of the NGC 2071 nebula are rich in Spitzer identified protostars relative to more evolved pre-main sequence stars (see Megeath et al. 2012), further evidence that the areas rich in PBRs have a younger age. 


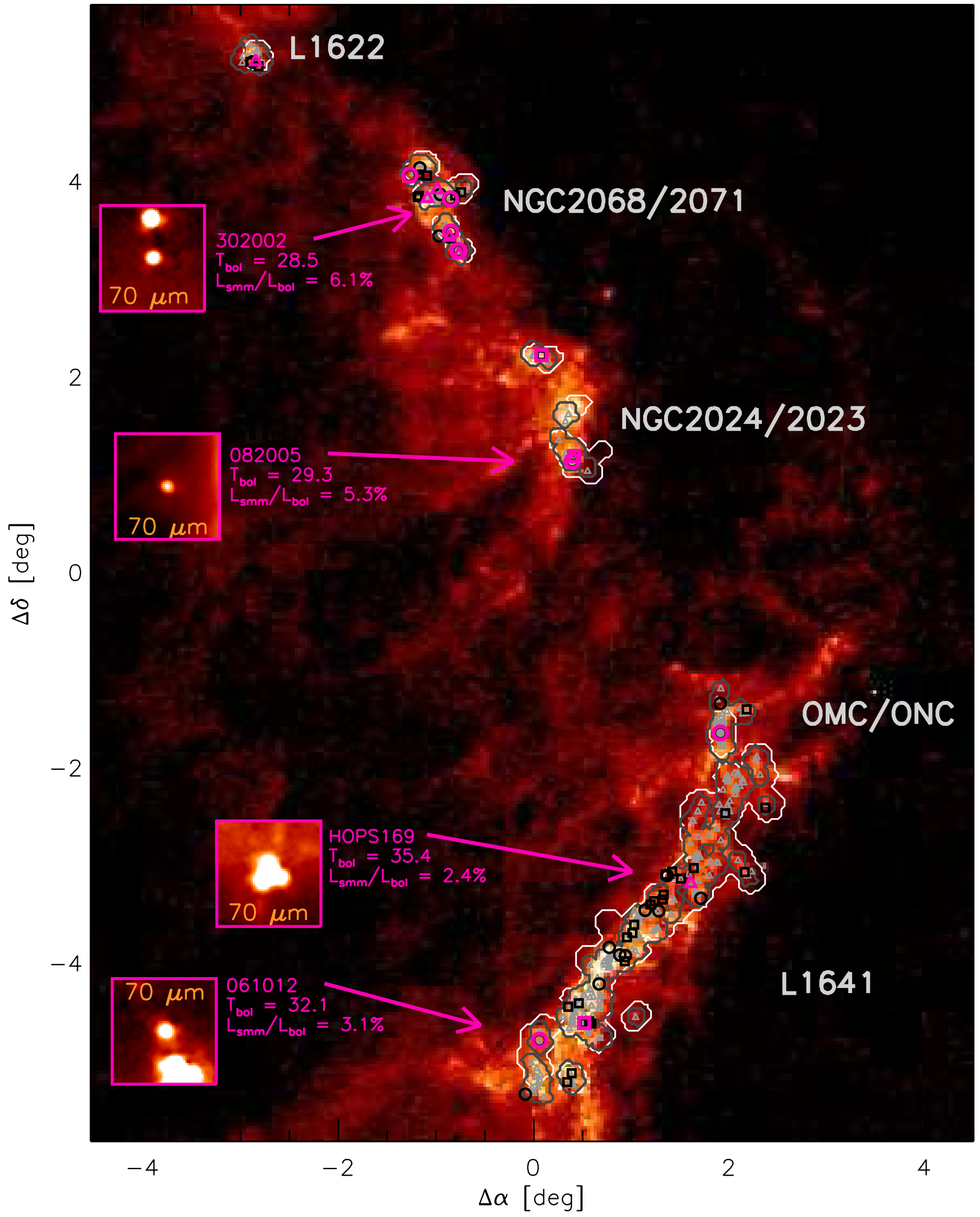

Figure 10. Distribution of red protostars overlaid on the extinction map of Orion. The dark gray contour shows the HOPS PACS coverage and the white contour shows the LABOCA $870 \mu \mathrm{m}$ coverage. The triangles (light gray) indicate the positions of the HOPS protostars while the black points (squares and circles) indicate the positions of the new candidate protostars. The PBRs are highlighted in fuchsia and the properties of four selected PBRs are shown along with their corresponding $1^{\prime} \times 1^{\prime}$ HOPS $70 \mu \mathrm{m}$ images. Orion B includes the prominent northern regions while Orion A includes the southern OMC/ONC and L1641 regions; note the large fractions of PBRs in NGC 2068/2071.

(A color version of this figure is available in the online journal.) 
Alternatively, the gas in the LBS23 and NGC 2023 clumps have dense gas-filling factors that are much higher than the other massive clumps (Lada et al. 1997); hence, the protostars in these regions may be forming in a very different birth environment. We will investigate the roles of non-coevality and environment in determining the percentage of PBRs in future work.

\section{DETERMINING THE PHYSICAL PROPERTIES OF PBRs THROUGH MODELS}

In this section we describe our analysis of the physical properties of the PBRs as inferred from their colors and SEDs. We first compare the 70/24 colors of the PBRs sample with those derived from a grid of models that adopt the solution for a rotating envelope undergoing collapse (Ulrich 1976; Terebey et al. 1984) with outflow cavities along the rotation axis of the envelope (Whitney et al. 2003b). This analysis puts a constraint on the minimum inner density of the protostellar envelope. Next, we compare the observed SEDs to model SEDs generated using the Hyperion (Robitaille 2011) radiative transfer code. This set of models assumes radial power-law gradients consistent with either a collapsing core with a constant infall rate or a static isothermal core. The models also encompass various combinations of internal and external heating. Given the prohibitively large computational time needed to explore the full range of parameter space using radiative transfer models, and given our inability to distinguish between models purely from five to six photometry points, we do not provide individual model fits to each protostar. Instead, we fit a single-temperature modified blackbody function to the observed SEDs at $70 \mu \mathrm{m}$ and longer wavelengths. The modified blackbody fits provide luminosities and an initial characterization of the envelope masses of the PBRs sample.

\subsection{Axisymmetric Models: Interpreting the 70/24 Color}

We begin our analysis by using a simplified version of the Ali et al. (2010) protostellar envelope model grid to predict observed fluxes and colors for comparison with our PBRs. The density distribution of these models is that of the collapse of a spherical cloud in uniform rotation (Ulrich 1976), which is the inner region of the Terebey et al. (1984) model of the collapse of the slowly rotating isothermal sphere. This model is then modified by the inclusion of outflow cavities of various shapes (Whitney et al. 2003a, 2003b). This schematic model envelope captures the dependence of the short wavelength $(24 \mu \mathrm{m}$ and $70 \mu \mathrm{m}$ ) fluxes on inclination due to rotation and bipolar cavities.

The model fluxes depend upon the mass infall rate, the angular momentum of the mass currently falling in, the outflow cavity properties, the inclination of the rotation axis to the line of sight (LOS), and the luminosity of the central source, as well as the assumed dust properties. The thermal emission of the dusty envelope does not depend directly on the mass infall rate but instead on the density of the envelope. The model assumes free-fall at a constant rate, which results in a density profile with shape $\rho \propto r^{-3 / 2}$ (Terebey et al. 1984). The overall scaling of the density is characterized by $\rho_{1}$, the density at $1 \mathrm{AU}$ in the limit of no rotation:

$$
\rho_{1}=7.4 \times 10^{-15}\left(\frac{\dot{M}_{\mathrm{env}}}{10^{-6} M_{\odot} \mathrm{yr}^{-1}}\right)\left(\frac{M_{*}}{0.5 M_{\odot}}\right)^{-1 / 2} \mathrm{~g} \mathrm{~cm}^{-3} .
$$

The envelope mass infall $\dot{M}_{\text {env }}$ rate is related to $\rho_{1}$ via the free-fall velocity, which in turn depends upon the unknown central mass $M_{*}$. The actual model density structure departs from $r^{-3 / 2}$ on small scales because of the angular momentum of the infalling material. This enters into the model through the parameter $R_{\text {disk }}$, the outer disk radius at which infalling material currently lands (see Ulrich 1976).

The rotation leads to a significant dependence of the SED on the inclination of the rotation axis relative to the LOS (Kenyon et al. 1993). This dependence is significantly enhanced by the inclusion of outflow cavities (Whitney et al. 2003b) which are assumed to be aligned along the rotation axis. Finally, the overall shape of the SED is only weakly affected by the luminosity of the central source ( $L_{*}$; Kenyon et al. 1993) and so this is easily scaled.

To roughly compare observed PBRs' colors with those predicted by our model grid, in Figure 11 we show the effects of varying the model inclination, envelope density, and cavity opening angle on the $70 / 24$ color and $70 \mu \mathrm{m}$ flux. As stated above, these model tracks are based on a simplified version of the Ali et al. (2010) model grid; we refer the reader to that publication for details. In brief, the model tracks that we consider here have the same fixed parameters as those listed in Table 1 of Ali et al. (2010), including a fixed central mass of $0.5 M_{\odot}$; in addition, we have fixed the cavity shape exponent to a value of $b=1.5$, and the envelope outer radius to $R_{\text {env, } \max }=1 \times 10^{4} \mathrm{AU}$. We have, however, expanded the envelope infall rate grid relative to the Ali et al. (2010) grid to larger values (up to $\dot{M}_{\text {env }}=10^{-3} M_{\odot} \mathrm{yr}^{-1}$ on a pseudologarithmic grid) and included a model with no envelope. As described above, for our model grid we assume that the envelope density falls off as $\rho(r) \propto r^{-3 / 2}$. Note that although our model grid is parameterized in terms of $\dot{M}_{\text {env }}$, with a fixed central mass of $M_{*}=0.5 M_{\odot}$, we will refer to $\rho_{1}$ throughout this section.

We note that the central masses of protostellar sources remain largely unconstrained observationally (however, see Tobin et al. 2012), and therefore may be lower than our assumed value. The effect of a lower central mass would be to lower $\dot{M}_{\text {env }}$ for a given value of $\rho_{1}$. For example, if the central mass were $0.2 M_{\odot}$, the infall rates reported for our models would be reduced by a factor of 0.6. The assumed central mass, however, does not change the value of $\rho_{1}$ corresponding to a given model SED. Furthermore, our small assumed disk radius does not strongly affect the trends shown here.

With this model grid we isolate the effects, albeit in a simplified fashion, of varying the model inclination (viewing angle to the protostar), envelope density $\left(\rho_{1}\right)$, and cavity opening angle $\left(\theta_{\mathrm{C}}\right)$ on the $70 \mu \mathrm{m}$ fluxes and $70 / 24$ colors. In Figure 11, we show model tracks through $70 \mu \mathrm{m}$ flux versus $70 / 24$ color space for high inclination $\left(87^{\circ}\right)$ viewing angles as a function of both $\theta_{\mathrm{C}}$ and envelope density $\left(\rho_{1}\right)$. By analyzing only the high inclination models, we obtain a lower limit on the envelope density required to reproduce the red 70/24 colors.

We assume a fixed disk accretion rate of $\dot{M}_{\text {disk }}=1.0 \times$ $10^{-8} M_{\odot} \mathrm{yr}^{-1}$, a disk outer radius of $R_{\text {disk, max }}=5 \mathrm{AU}$, and a central source luminosity of $1 L_{\odot}$. Increasing $\dot{M}_{\text {disk }}$ and $R_{\text {disk, max }}$ drives the models to both bluer $70 / 24$ colors and brighter $70 \mu \mathrm{m}$ fluxes, while decreasing the inclination drives the models to bluer 70/24 colors. Therefore, we find that no model in our grid can explain 70/24 colors with envelope densities less than log $\rho_{1} /\left(\mathrm{g} \mathrm{cm}^{-3}\right) \sim-13.4$ (or equivalently an envelope infall rate of $\left.\dot{M}_{\text {env }}=5.4 \times 10^{-6} M_{\odot} \mathrm{yr}^{-1}\right)$. While models with larger values of $\rho_{1}$ and other combinations of parameters can be found for bluer $70 / 24$ colors, this analysis sets an approximate lower limit on the expected envelope densities of sources with $70 / 24>1.65$ 

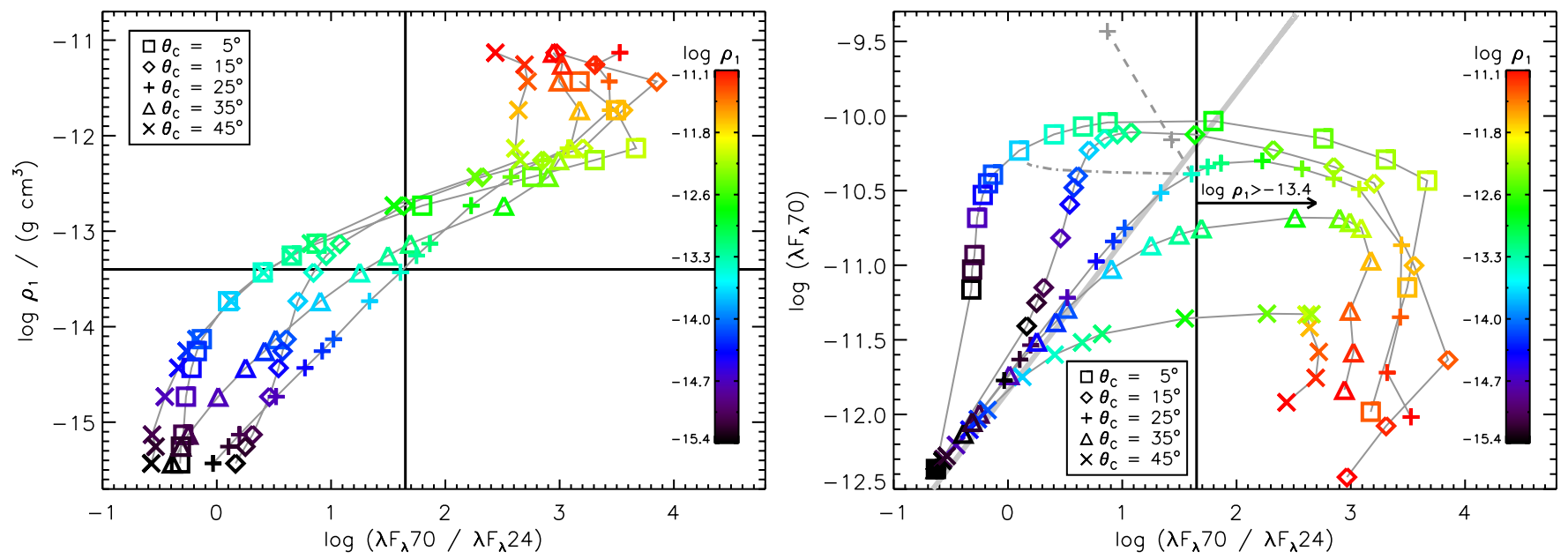

Figure 11. Left: dependence of the model $70 \mu \mathrm{m}$ to $24 \mu \mathrm{m}$ flux ratio for high inclination orientations $\left(87^{\circ}\right)$ on envelope density $\left(\rho_{1}\right)$ for the five cavity opening angles in our grid. These model tracks assume an internal luminosity of $1.05 L_{\odot}$. The 70/24 color selection is indicated with the vertical black line, while the corresponding $\log \rho_{1} /\left(\mathrm{g} \mathrm{cm}^{-3}\right) \sim-13.4$ (corresponding to an envelope infall rate of $\left.\dot{M}_{\mathrm{env}}=7.5 \times 10^{-6}\right)$ threshold is indicated with a horizontal black line. Right: model $70 \mu \mathrm{m}$ flux vs. $70 \mu \mathrm{m}$ to $24 \mu \mathrm{m}$ flux ratio for high inclination orientations $\left(87^{\circ}\right)$. For the model with a cavity opening angle of $\theta_{\mathrm{C}}=25^{\circ}(+\operatorname{symbols})$ and $\log \rho_{1} /\left(\mathrm{g} \mathrm{cm}{ }^{-3}\right) \sim-13.4$, we show the effect of increasing the internal luminosity by two orders of magnitude with the dashed gray curve, while the effect of decreasing the inclination to $18^{\circ}$ is illustrated with the dot-dashed curve. All models with higher assumed values of luminosity and lower inclination will have envelopes that are denser than log $\rho_{1} /\left(\mathrm{g} \mathrm{cm}^{-3}\right) \sim-13.4$, the median value of $\rho_{1}$ in our model grid; sources with redder 70 to $24 \mu \mathrm{m}$ colors cannot be explained by envelopes that are less dense than this threshold. The thick light gray line corresponds to a $24 \mu \mathrm{m}$ limit of $7 \mathrm{mag}$, the imposed Spitzer protostar magnitude limit for identification of protostars.

(A color version of this figure is available in the online journal.)

of $\log \rho_{1} /\left(\mathrm{g} \mathrm{cm}^{-3}\right) \gtrsim-13.4$. That is, high source inclinations alone cannot explain the red 70/24 colors of the PBRs, which also require dense envelopes.

We expect that this value of $\rho_{1}$ should lie well within the Class 0 range; e.g., Furlan et al. (2008) found for a sample of 22 Class I sources in Taurus that $\log \rho_{1} /\left(\mathrm{g} \mathrm{cm}^{-3}\right) \leqslant-13.2$, while over half had $\rho_{1}$ values that are lower than $\log \rho_{1} /\left(\mathrm{g} \mathrm{cm}^{-3}\right)=$ -13.4. Note that the Furlan et al. (2008) data set included far better sampling of the source SEDs and, most critically, Spitzer IRS spectroscopy, allowing for a more robust estimate of the parameters of sources in their sample. In contrast, our SEDs generally are envelope dominated with few sources having robust detections shortward of $24 \mu \mathrm{m}$. Therefore, any estimate of the value of the envelope density will be necessarily imprecise and have a back of the envelope character. That said, our comparison with the model grid and previous derived values of the envelope density leads us to conclude that the $70 / 24>1.65$ color cut, while not uniformly selecting a unique envelope density threshold, will preferentially select Class 0 sources with $\log \rho_{1} /\left(\mathrm{g} \mathrm{cm}^{-3}\right) \gtrsim-13.4$, irrespective of source inclination.

Having demonstrated that the very red 70/24 colors require large values of $\rho_{1}$, as expected for SEDs peaking at long wavelengths, we proceed by analyzing the PBRs in the context of more simplified models. In what follows, we carry out two independent analyses of the source SEDs: a qualitative model image comparison using 1D models generated with the Hyperion (Robitaille 2011) radiative transfer code, and modified blackbody fitting to the observed SEDs at $70 \mu \mathrm{m}$ and longer wavelengths. We attempt to maintain consistency by using similar envelope dust models throughout the analysis presented here. For the Hyperion model image analysis we use the Ormel et al. (2011) opacities. These opacities are similar to the commonly assumed Ossenkopf \& Henning (1994, "OH5"; see below) opacities but include both the scattering and absorption components at short wavelengths, needed for radiative transfer calculations. Specifically, we use the "icsgra2" Ormel et al.
(2011) model opacities, which include icy silicates and bare graphites, with a coagulation time of $0.1 \mathrm{Myr}$. We assume the dust model from Ossenkopf \& Henning (1994) for the longwavelength-modified blackbody fits, specifically, their "OH5" opacities, corresponding to Column 5 of their Table 1 . These opacities reflect grains having thin ice mantles with $10^{5}$ years of coagulation time at an assumed gas density of $10^{6} \mathrm{~cm}^{-3}$.

\subsection{Spherically Symmetric Models: Comparison to Photometry Derived from Model Images}

We use the Hyperion (Robitaille 2011) Monte Carlo radiative transfer code to investigate some basic properties of the PBRs. We run a series of spherically symmetric models under a range of very simple assumptions, and produce simulated images. As stated above, the dust model we assume is that of Ormel et al. (2011, "icsgra2"). To explore which model assumptions might be reasonable for analyzing the properties of the reddest sources in Orion, we investigate a few limiting model scenarios. We therefore disregard the details of the individual source SEDs of the entire red sample and qualitatively compare the SEDs of the two sources with the smallest and largest values of $L_{\mathrm{bol}}$ and $T_{\text {bol }}$ (sources 091016 and HOPS358, respectively; see Table 8) with photometry derived from model images.

We generate a series of model images that fall into four classes: (1) a starless core at a constant temperature of $10 \mathrm{~K}$ (referred to here as the "core" model); (2) a starless core with an isotropic external radiation field ("core+external"); (3) a core with an internal source ("star" model); and finally (4) a core with both an internal source and an isotropic external radiation field ("star+external"). For each of these classes of models, we test a range of density profile shapes and density normalizations. For the density profile shape we assume two values, $\alpha=1.5,2.0$, where $\alpha$ is the radial density profile power-law index: $\rho(r) \propto$ $r^{-\alpha}$. For the absolute value of the (gas) density normalization at $1 \mathrm{AU}$ we assume five values, $\log \left(\rho_{1 \mathrm{AU}} /\left(\mathrm{g} \mathrm{cm}^{-3}\right)\right)=-10$ to -14 , in steps of $\delta \log \left(\rho_{1 \mathrm{AU}} /\left(\mathrm{g} \mathrm{cm}^{-3}\right)\right)=1.0$. For models 
Table 8

Orion PBRs and Comparison Class 0 Sources: Observed Properties and Modified Blackbody Fit Parameters

\begin{tabular}{|c|c|c|c|c|c|c|c|}
\hline Source & $\begin{array}{l}T_{\text {bol }} \\
(\mathrm{K})\end{array}$ & $\begin{array}{l}L_{\text {bol }} \\
\left(L_{\odot}\right)\end{array}$ & $\begin{array}{c}L_{\mathrm{smm}} / L_{\mathrm{bol}}{ }^{\mathrm{a}} \\
(\%)\end{array}$ & $\begin{array}{c}T_{\mathrm{MBB}}{ }^{\mathrm{b}} \\
(\mathrm{K})\end{array}$ & $\begin{array}{c}L_{\mathrm{MBB}}{ }^{\mathrm{b}} \\
\left(L_{\odot}\right)\end{array}$ & $\begin{array}{c}M_{\mathrm{MBB}} \mathrm{b}, \mathrm{c} \\
\left(M_{\odot}\right)\end{array}$ & $\begin{array}{c}\lambda_{\text {peak,MBB }}{ }^{\mathrm{b}} \\
(\mu \mathrm{m})\end{array}$ \\
\hline $061012^{d}$ & $32.1 \pm 0.9$ & $0.75 \pm 0.06$ & 3.1 & $19.2 \pm 0.4$ & $0.68 \pm 0.07$ & $0.20 \pm 0.04$ & 133 \\
\hline 119019 & $34.4 \pm 0.9$ & $1.56 \pm 0.14$ & 2.6 & $19.9 \pm 0.4$ & $1.35 \pm 0.11$ & $0.32 \pm 0.06$ & 127 \\
\hline HOPS169 & $35.4 \pm 0.9$ & $4.50 \pm 0.42$ & 2.4 & $20.4 \pm 0.5$ & $3.85 \pm 0.41$ & $0.81 \pm 0.20$ & 127 \\
\hline $019003^{d}$ & $33.6 \pm 1.1$ & $3.16 \pm 0.29$ & 1.1 & $21.4 \pm 0.8$ & $1.52 \pm 0.30$ & $0.24 \pm 0.18$ & 121 \\
\hline $082005^{d}$ & $29.3 \pm 0.8$ & $1.02 \pm 0.11$ & 5.4 & $17.0 \pm 0.4$ & $1.02 \pm 0.13$ & $0.61 \pm 0.15$ & 151 \\
\hline HOPS372 & $36.9 \pm 1.0$ & $4.90 \pm 0.45$ & 1.9 & $21.3 \pm 0.6$ & $3.96 \pm 0.43$ & $0.65 \pm 0.17$ & 121 \\
\hline 082012 & $32.2 \pm 0.9$ & $6.27 \pm 0.65$ & 3.8 & $18.6 \pm 0.4$ & $5.89 \pm 0.71$ & $2.20 \pm 0.52$ & 139 \\
\hline 090003 & $36.0 \pm 0.8$ & $2.71 \pm 0.24$ & 1.8 & $21.4 \pm 0.4$ & $2.06 \pm 0.15$ & $0.33 \pm 0.06$ & 121 \\
\hline HOPS358 & $44.3 \pm 0.9$ & $30.6 \pm 2.21$ & 0.6 & $27.2 \pm 1.0$ & $19.5 \pm 1.23$ & $0.78 \pm 0.14$ & 88. \\
\hline $091015^{\mathrm{d}}$ & $30.9 \pm 0.9$ & $0.81 \pm 0.07$ & 3.3 & $18.7 \pm 0.4$ & $0.72 \pm 0.06$ & $0.24 \pm 0.04$ & 136 \\
\hline $091016^{\mathrm{d}}$ & $29.1 \pm 0.9$ & $0.65 \pm 0.06$ & 4.1 & $17.9 \pm 0.3$ & $0.61 \pm 0.06$ & $0.26 \pm 0.05$ & 142 \\
\hline HOPS373 & $36.0 \pm 0.9$ & $5.20 \pm 0.48$ & 2.3 & $20.2 \pm 0.4$ & $4.21 \pm 0.32$ & $0.93 \pm 0.16$ & 127 \\
\hline $093005^{\mathrm{d}}$ & $30.8 \pm 0.9$ & $1.71 \pm 0.15$ & 3.4 & $18.8 \pm 0.4$ & $1.57 \pm 0.15$ & $0.52 \pm 0.10$ & 136 \\
\hline $302002^{d}$ & $28.6 \pm 0.9$ & $0.84 \pm 0.10$ & 6.1 & $16.2 \pm 0.3$ & $0.82 \pm 0.11$ & $0.63 \pm 0.15$ & 157 \\
\hline HOPS359 & $39.3 \pm 0.9$ & $12.6 \pm 1.00$ & 1.3 & $23.4 \pm 0.7$ & $9.56 \pm 0.84$ & $0.91 \pm 0.23$ & 115 \\
\hline HOPS341 & $36.3 \pm 1.1$ & $3.62 \pm 0.37$ & 3.1 & $19.1 \pm 0.5$ & $3.16 \pm 0.35$ & $0.97 \pm 0.24$ & 133 \\
\hline $097002^{\mathrm{d}}$ & $33.4 \pm 0.9$ & $1.14 \pm 0.11$ & 2.8 & $19.4 \pm 0.5$ & $0.99 \pm 0.11$ & $0.27 \pm 0.07$ & 130 \\
\hline HOPS354 & $37.4 \pm 0.8$ & $7.53 \pm 0.74$ & 1.9 & $21.0 \pm 0.4$ & $5.84 \pm 0.45$ & $1.05 \pm 0.19$ & 124 \\
\hline B $335^{\mathrm{e}}$ & $40.3 \pm 1.3$ & $0.55 \pm 0.04$ & 2.6 & $20.0 \pm 0.7$ & $0.47 \pm 0.04$ & $0.11 \pm 0.02$ & 130 \\
\hline $\mathrm{CB} 130^{\mathrm{e}}$ & $55.2 \pm 3.3$ & $0.22 \pm 0.01$ & 10.9 & $12.7 \pm 0.5$ & $0.17 \pm 0.01$ & $0.55 \pm 0.11$ & 199 \\
\hline CB $244-S M M 1^{\mathrm{e}}$ & $63.2 \pm 2.6$ & $1.72 \pm 0.14$ & 1.8 & $20.3 \pm 0.8$ & $1.10 \pm 0.11$ & $0.24 \pm 0.04$ & 127 \\
\hline $\mathrm{CB} 68^{\mathrm{e}}$ & $49.2 \pm 1.4$ & $0.68 \pm 0.06$ & 1.9 & $19.8 \pm 1.1$ & $0.42 \pm 0.05$ & $0.10 \pm 0.02$ & 130 \\
\hline VLA $1623-243^{\mathrm{f}}$ & $34.0 \pm 1.2$ & $2.82 \pm 0.32$ & 2.4 & $21.3 \pm 0.5$ & $2.55 \pm 0.24$ & $0.48 \pm 0.09$ & 124 \\
\hline
\end{tabular}

Notes.

a Our $L_{\mathrm{smm}} / L_{\text {bol }}$ may be underestimated as the $L_{\mathrm{smm}}$ values are measured from the modified blackbody fits to the SEDs (see the text).

$\mathrm{b}$ Best-fit modified blackbody parameters; the parameters shown here are derived from fitting the $\lambda \geqslant 70 \mu \mathrm{m}$ SED points. Excluding the $70 \mu \mathrm{m}$ point and fitting only the $\lambda \geqslant 100 \mu \mathrm{m}$ points systematically increases the derived masses by $20 \%$ on average, decreases the temperature by $5 \%$ on average, and decreases the luminosity by $7 \%$ on average.

c The masses derived here will increase by a factor of $\sim 4$ if we assume an ISM-type dust model (Draine \& Lee 1984). Furthermore, we assume a gas-to-dust ratio of 110 (see the text).

d The values of $T_{\text {bol }}$ and $L_{\text {bol }}$ for these sources should be considered upper limits because we include the $24 \mu \mathrm{m}$ upper limits in our analysis for these sources. e Additional well-studied isolated Class 0 sources from Launhardt et al. (2013) and Stutz et al. (2010) are shown for comparison; the distances to these sources lie between $100 \mathrm{pc}$ and $250 \mathrm{pc}$.

f SED from J. Green and DIGIT team (2012, private communication) and J. Green et al. (in preparation).

with external heating, the bolometric strength of the interstellar radiation field (ISRF) is set to the value from Mathis et al. (1983) at the solar neighborhood $\left(4 \pi J_{v}=0.0217 \mathrm{erg} \mathrm{cm}^{-2} \mathrm{~s}^{-1}\right)$. The spectrum of the radiation field is assumed to be that at the solar neighborhood from Porter \& Strong (2005), but reddened by $A_{V}=10$ using the Kim et al. (1994) extinction law. The ISRF model includes contributions from the stellar, PAH, and FIR thermal emission. The inner radius of the core is set to the radius at which the dust sublimates, assuming a sublimation temperature of $1600 \mathrm{~K}$, while the outer radius is set to $1 \mathrm{pc}$. The central source is taken to have $10 L_{\odot}$ and a spectrum given by a Planck function at the effective temperature of the Sun $(5778 \mathrm{~K})$. The choice of the stellar temperature is arbitrary, and is unimportant for the modeling presented here, since all sources are deeply embedded and all stellar radiation is reprocessed-only the total bolometric luminosity is important (see, e.g., Johnston et al. 2012, for a discussion of the $R_{\text {star }}$ and $T_{\text {star }}$ degeneracy).

The high levels of spatial filtering caused by our adopted aperture photometry scheme require us to assume such a central source luminosity to roughly match the flux levels in the observed SEDs. Furthermore, while a 1 pc sized envelope is larger than usually assumed, the high levels of spatial filtering inherent in the aperture photometry cause us to be insensitive to structure on scales larger than the assumed aperture sizes.
The model images have a resolution of $1^{\prime \prime}$ pixel ${ }^{-1}$, or $420 \mathrm{AU}$ at our assumed distance. We convolve these images with the azimuthally averaged PSFs provided by Aniano et al. (2011), except in the case of the SABOCA $350 \mu \mathrm{m}$ and LABOCA $870 \mu \mathrm{m}$ images. These wavelengths are convolved with Gaussian PSFs with FWHMs equal to $77^{\prime \prime} 4$ and $19^{\prime \prime}$, respectively, i.e., the nominal beam sizes for our observations. All model image photometry is then performed on the convolved model images using the same aperture and sky annulus parameters as those applied to the data. The use of such photometric aperture parameters can cause substantial spatial filtering due to the small sizes of the apertures relative to the beam sizes and the extent of the core emission (see below).

We show our extracted model SEDs in Figure 12 and a subset of the corresponding model images in Figure 13. In general, we find that models without internal sources are very unlikely to match the observed PBRs properties, on the basis that their SEDs are less luminous and peak at longer wavelengths than the observed SEDs for all density profile shapes that we assume; see Figure 12, top panels. While the "core+external" models (top right panel) suffer from severe spatial filtering, it is unlikely that such models will well represent the data as these SEDs tend to also peak at longer wavelengths. For the two classes of models with internal sources we find better agreement with the data; see lower panels of Figure 12. While steeper envelope profiles 

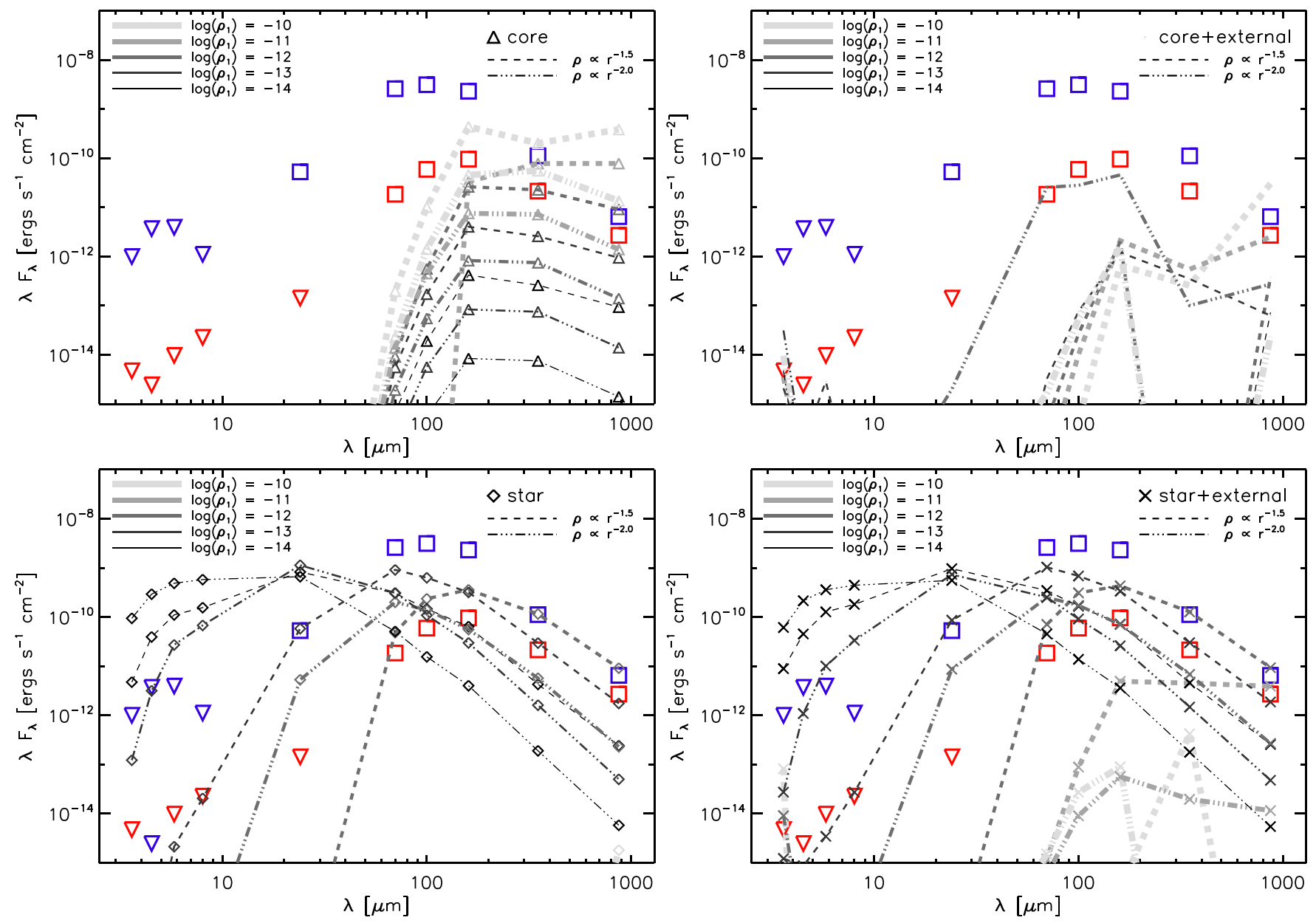

Figure 12. All panels show the SEDs of HOPS358 (blue: $L_{\mathrm{bol}}=30.6 L_{\odot}$ and $T_{\mathrm{bol}}=44.2 \mathrm{~K}$ ) and 091016 (red: $L_{\mathrm{bol}}=0.65$ and $T_{\mathrm{bol}}=29 \mathrm{~K}$ ), the extrema of the $L_{\mathrm{bol}}$ and $T_{\mathrm{bol}}$ distributions for the reddest sources, compared to spherical models. The two line styles indicate the different assumptions for the density profile shape while the shade of gray indicates the different model density $\rho_{1}$ values. The four panels correspond to the four different models that we test (see the text) - top left: the "core" model, a starless core; top right: the "core+external" model, a starless core with external heating; bottom left: the "star" model, a core with an internal source; and finally, bottom right: the "star+external" model, consisting of a core with an internal source that is irradiated by an external radiation field. Severe spatial filtering due to our aperture photometry on the model images can be seen most notably in the two externally heated models (left column).

(A color version of this figure is available in the online journal.)
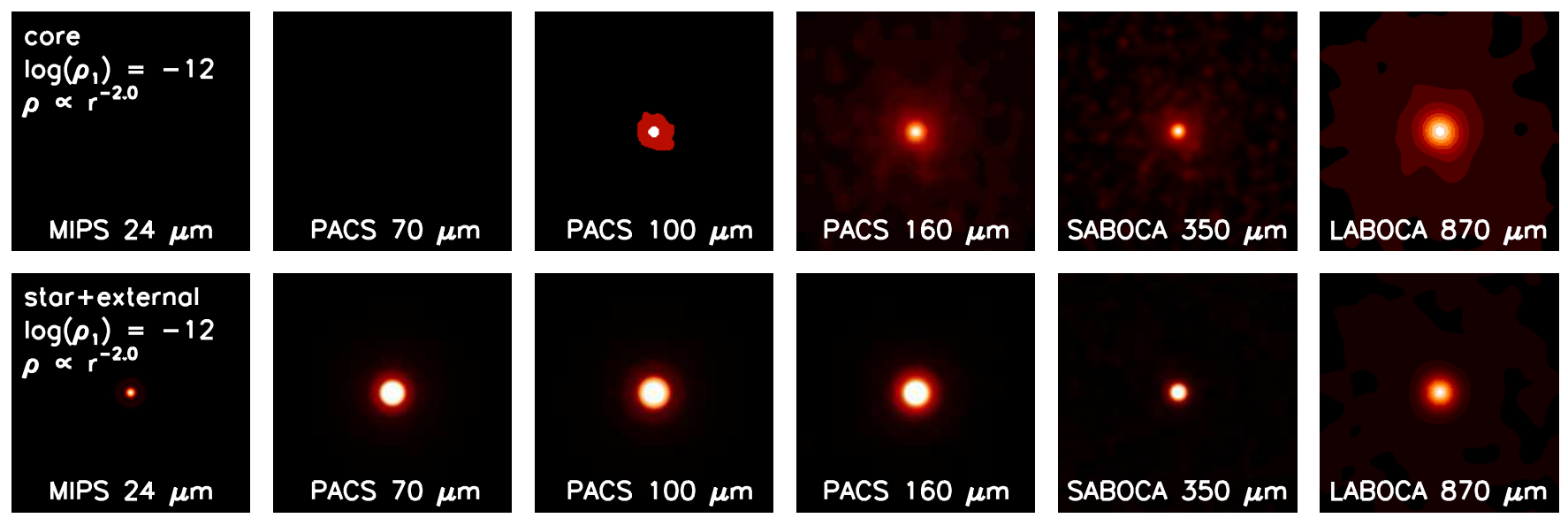

Figure 13. Convolved model images at the indicated wavelengths, shown on a log scale with the same minimum and maximum flux levels for all panels to illustrate the shape of the model SEDs. Each image is 200 " (or 84,000 AU) on a side. The top row shows the "core" model images, with $\log \left(\rho_{1}\right)=-12$ and a density profile shape of $\rho \propto r^{-2.0}$, corresponding to the top left panel in Figure 12. The bottom row shows the "star+external" model, with the same density parameters as the top row, corresponding to the bottom right panel in Figure 12.

(A color version of this figure is available in the online journal.) 
may imply somewhat higher envelope densities, the range in plausible densities for $\alpha=1.5$ is $\log \rho_{1} \sim-12$ to -13 , while for $\alpha=2.0$ values of $\log \rho_{1} \sim-12$ roughly agree with the shapes of the observed SEDs. We note that we find very little difference between the "star+external" and the "star" model in the $\lambda \lesssim 160 \mu \mathrm{m}$ regime, possibly indicating that our assumed ISRF strength relative to the assumed internal source luminosity may be underestimated compared to what we might expect to find regions like Orion.

\subsection{Modified Blackbody Fits to the PBRs}

An individual detailed fit to each protostar is beyond the scope of this work considering the vast amount of parameter space needed to model protostellar SEDs (i.e., source luminosity, envelope density, envelope rotation, outflow cavity geometry, external heating, outer envelope structure). Furthermore, unlike most of the HOPS protostars, whose properties can be constrained from a combination of far-IR photometry, $5 \mu \mathrm{m}$ to $40 \mu \mathrm{m}$ Spitzer/IRS spectra, and Hubble near-IR imaging (Fischer et al. 2010, 2012), the properties of the SEDs must currently be derived from five to six photometry points at long wavelengths. While the above modeling and analysis shows that the internal source is important, the longer wavelength fluxes are probing the bulk of the envelope mass, expected to mostly be at a single temperature. Furthermore, for density profiles in the range of $\rho(r) \propto r^{-3 / 2}$ or $r^{-2}$, as assumed above, we expect that most of the envelope will be located at large radii. We therefore perform modified blackbody fits to the $\lambda \geqslant 70 \mu \mathrm{m}$ SEDs listed in Tables 4 and 5. For this analysis, we use the beam flux measurements for the sub-millimeter $350 \mu \mathrm{m}$ and $870 \mu \mathrm{m}$ portion of the SED. The results of the analysis are presented in Table 8 and the model SEDs are plotted with the data in Figure 9.

Before fitting the long-wavelength SEDs of the sources, we apply color corrections to the Herschel $70 \mu \mathrm{m}, 100 \mu \mathrm{m}$, and $160 \mu \mathrm{m}$ fluxes. Following Launhardt et al. (2013), these photometric color corrections have been derived iteratively from the slopes of the PACS SEDs, using polynomial fits to the values in Table 2 of the PACS calibration release note "PACS Photometer Passbands and Colour Correction Factors for Various Source SEDs" from 2011 April 12. The color corrections for the APEX data are assumed to be negligible.

The form of the modified blackbody function is given by

$$
S_{v}=\Omega B_{v}\left(v, T_{\mathrm{d}}\right)\left(1-e^{-\tau(v)}\right),
$$

where $\Omega$ is the solid angle of the emitting element, $B_{v}\left(T_{\mathrm{d}}\right)$ is the Planck function at a dust temperature $T_{\mathrm{d}}$, and $\tau(\nu)$ is the optical depth at frequency $v$. Here, the optical depth is given by $\tau(v)=N_{\mathrm{H}} m_{\mathrm{H}} R_{\mathrm{gd}}^{-1} \kappa(v)$, where $N_{\mathrm{H}}=2 \times N\left(\mathrm{H}_{2}\right)+N(\mathrm{H})$ is the total hydrogen column density, $m_{\mathrm{H}}$ in the proton mass, $\kappa_{v}$ is the assumed dust opacity law from Ossenkopf \& Henning (1994), and $R_{\mathrm{gd}}$ is the gas-to-dust ratio, assumed to be 110 (Sodroski et al. 1997). The best-fit total masses $M_{\text {tot }}$ reported in Table 8 have been multiplied by an additional factor of 1.36 to account for helium and metals. Furthermore, in Table 8 we also report the peak wavelength of the best-fit modified blackbody model. Finally, we estimate $L_{\mathrm{smm}}$ from the model SED, where $L_{\mathrm{smm}}$ is integrated over $\lambda \geqslant 350 \mu \mathrm{m}$.

If a given source SED has coverage over fewer than four longwavelength points, we do not fit a model to the SED. While all the PBRs' sources satisfy this criterion, all the new candidate protostars and HOPS sources do not (see Section 5.2) and are therefore not fitted. The errors on $T_{\mathrm{d}}, M_{\text {tot }}$, and the thermal component of the luminosity $\left(L_{\mathrm{MBB}}\right)$ are estimated through a straightforward Monte Carlo method. ${ }^{14}$ For each source we generate 2000 synthetic SEDs drawn from a normal density with mean and standard deviation equal to those of the measured SED at each wavelength. We then fit each synthetic SED. The reported error is equal to the standard deviation of the resulting distribution of each parameter. These errors do not include systematics introduced by, e.g., our dust model assumption or variation in the gas-to-dust ratio.

We show the modified blackbody fit results, along with the SEDs of the PBRs, in Figure 9. The resulting best-fit mass, luminosity, and temperature is also indicated for each source. The model fits the data surprising well considering that significant temperature gradients in the envelope are expected. Furthermore, in all cases, the $24 \mu \mathrm{m}$ point, when detected, has a much higher flux level than the modified blackbody model. We interpret this discrepancy as strong evidence for internal heating by a protostar.

Excluding the $70 \mu \mathrm{m}$ point and fitting only the $\lambda \geqslant 100 \mu \mathrm{m}$ SED has a minor effect on the resulting parameter values. Without $70 \mu \mathrm{m}$, the masses systematically increase by $40 \%$, the temperatures decrease by $5 \%$, and the luminosities decrease by $7 \%$. This small effect may be understood by the fact that the $100 \mu \mathrm{m}$ fluxes are well correlated with the $70 \mu \mathrm{m}$ fluxes for this sample, tracing similar material near the protostars. The dust temperatures probed at $100 \mu \mathrm{m}$ and $70 \mu \mathrm{m}$ are not dramatically different since the $\tau=2 / 3$ surfaces are not extremely different between the two wavelengths. From Hartmann (2009), the radius of the $\tau=2 / 3$ surface can be roughly approximated as $r_{\lambda} \propto \kappa_{\lambda}^{2}$; this relation implies that the radii of the $\tau=2 / 3$ surfaces for the two wavelengths is $r_{70} / r_{100} \sim 2.5$.

Our best-fit modified blackbody model always underestimates the observed $870 \mu \mathrm{m}$ flux of all sources. The model sub-millimeter SEDs are always bluer than the observed SEDs. We find that the discrepancy is at the $0.83 \pm 0.26 \mathrm{Jy}$ level (or a factor of $\sim 3$ excess), where the error bar represents the standard deviation in the residual distribution. It is likely that this discrepancy is dominated by the larger beam size of the $870 \mu \mathrm{m}$ observations which has the effect of mixing the source flux with that of the surrounding cold and possibly high-column environment. Contamination to the $870 \mu \mathrm{m}$ flux by disk emission may also increase this discrepancy. Jørgensen et al. (2009) find average disk masses of $\sim 0.13 M_{\odot}$ (with a large scatter) across their sample of Class 0 sources. The sources in their sample that are comparable to our PBRs, however, are those with the lowest values of $T_{\mathrm{bol}}$. For reference, they found that IRAS4A1, with a $T_{\text {bol }}=43 \mathrm{~K}$, has the largest disk mass of $0.46 M_{\odot}$ in their sample; on the other hand, L1157, with a similar $T_{\text {bol }}=42 \mathrm{~K}$, has a disk mass about a factor of four smaller. We estimate that a $30 \mathrm{~K}$ disk of $0.5 M_{\odot}$ would contribute $\sim 0.6$ Jy to the beam flux at $870 \mu \mathrm{m}$ (assuming Ossenkopf \& Henning 1994 dust opacities, as above). Therefore, disk emission could indeed contribute to the observed $870 \mu \mathrm{m}$ flux but further detailed observations at high resolution are needed to disentangle the envelope component from the possible disk emission. However, we do not find an $870 \mu \mathrm{m}$ discrepancy in the analysis of model images presented in the previous section. This indicates that large disk masses may not be necessary to explain the sub-millimeter fluxes. We

\footnotetext{
14 "Offered the choice between mastery of a five-foot shelf of analytical statistics books and middling ability at performing statistical Monte Carlo simulations, we would surely choose to have the latter skill.” Press et al. (1992,
} Numerical Recipes, p. 686). 
therefore emphasize that the disk masses inferred here from the $870 \mu \mathrm{m}$ excess should be regarded only as upper limits; further detailed investigation into the disk properties of our sources is deferred to future work.

Independent of these issues, it is clear that a more accurate treatment of the data would require all images to be convolved to a matched resolution; however, this approach would have the effect of causing non-detections for a majority of sources at the shorter wavelengths due to the relatively large limiting beam size of our data set $\left(\sim 19^{\prime \prime}\right.$ at $\left.870 \mu \mathrm{m}\right)$. Homogeneously extracted SEDs are therefore not feasible for this data set as a whole. Nevertheless, we test the effects of convolving the data before extracting the SEDs. We choose PBR 119019 as a test source because it is isolated and has approximately median values for the best-fit modified blackbody temperature, luminosity, and mass. Ignoring the $870 \mu \mathrm{m}$ data, this source is clearly detected at $70 \mu \mathrm{m}, 100 \mu \mathrm{m}, 160 \mu \mathrm{m}$, and $350 \mu \mathrm{m}$. For these four wavelengths, the largest beam size of $\sim 12^{\prime \prime}$ corresponds to the $160 \mu \mathrm{m}$ data. We therefore convolve the $70 \mu \mathrm{m}, 100 \mu \mathrm{m}$, and $350 \mu \mathrm{m}$ data to a resolution matching the $160 \mu \mathrm{m}$ observations and extract a beam-smoothed SED. We then fit this SED in the same way as described above. Compared to the non-convolved SED modified blackbody fitting results, we find that the temperature decreases by $\sim 4 \%$, the luminosity increases by $\sim 10 \%$, and the mass in the thermal component increases by $\sim 30 \%$. These systematic shifts are similar to but somewhat larger than the errors quoted in Table $8(\sim 2 \%$ on the temperature, $\sim 10 \%$ on the luminosity, and $\sim 30 \%$ on the mass). We note, however, that the errors quoted in Table 8 are purely random and do not include any systematic component. We therefore conclude that extracting SEDs from images matched to a resolution of $\sim 12^{\prime \prime}$ would not greatly affect our results.

Modified blackbody fits provide a somewhat limited means of analysis of our sources since the model assumes a single temperature and density along the LOS for the emitting material. We expect that the assumption of a single LOS temperature will cause an underestimate of the source masses (e.g., Nielbock et al. 2012; Launhardt et al. 2013). However, radiative transfer models have large ambiguities in the assumed source temperature and density structure, leading to mass estimates that are strongly model-dependent. Furthermore, the dust law that is assumed will introduce significant uncertainties into the derived masses, irrespective of the analysis method that is implemented. For example, the masses listed in Table 8 increase by a factor of $\sim 4$ on average when we assume Draine \& Lee (1984) $R_{V}=3.1$ interstellar medium (ISM) like dust. These issues indicate that the masses derived here represent lower limits to the true envelope masses. Nevertheless, we consider the modified blackbody fits to the measured photometry to provide the most robust estimates of the mass that we currently have.

We note that with only five SED flux points at best, fitting a multiple component (modified) blackbody model cannot be justified. Since most of the mass is located at relatively large scales and expected to have cold temperatures, excluding the warmer shorter wavelength data arising from inner material will not significantly increase the masses we derive. The modified blackbody fits thus provide an approximate measurement of the optical-depth-averaged gross properties of the envelopes being investigated. These issues point to the need for a more sophisticated modeling approach that will be carried out in future work.

\section{DISCUSSION}

As seen in Figure 11, the observed 70/24 colors of a protostar can be driven toward redder values through various strongly degenerate parameters. For example, the total column of material along the LOS toward a given protostar can have multiple contributions: the attenuation of the mid-IR emission by dense foreground material, the density of the envelope, the amount of envelope flattening, the opening angle of the outflow cavity, and the source inclination. Furthermore, the assumed model central protostar mass remains largely unconstrained by observations to date and can affect the interpretation of the $70 / 24$ colors.

Foreground extinction can have various contributions, such as intervening dust between the observer and the cloud and dense material associated with the cloud itself, such as filamentary material. Of these two components, the first is expected to be relatively small, while the latter can be expected to vary from source to source by relatively large amounts, with a corresponding effect on the observed colors. For example, some PBRs are located in filamentary regions (e.g., Figures 7 and 8), while others appear more isolated (e.g., see Appendix B for Figure 20). We have estimated the effects of foreground extinction levels up to a level of $A_{V}=40 \mathrm{mag}$, and find that the values of $L_{\mathrm{bol}}, L_{\mathrm{smm}}$, and $T_{\mathrm{bol}}$ are not significantly affected.

On the other hand, the effects of source inclination are not as straightforward to assess. When considering the presence of flattened rotating envelopes, disks, and outflow cavities, the source inclination will have a large effect on the observed source SED, as illustrated by the model tracks shown in Figure 11 (see also, e.g., Whitney et al. 2003b; De Buizer et al. 2005; Offner et al. 2012).

Therefore, it appears that the very red PBRs can be explained by multiple effects that all result in increasingly red observed $70 / 24$ colors. These very red colors may be driven by elevated envelope densities (or equivalently, $\dot{M}_{\text {env }}$ ), high source inclinations, or elevated levels of extinction associated with structures larger than the envelope-protostar system. The current data and SED coverage do not allow us to break these degeneracies conclusively. Furthermore, we consider it likely that the red observed colors are not driven by any single cause, but instead are the result of several causes.

We have, however, designed our PBR selection to find the densest envelopes in Orion (cf. Figure 11). Furthermore, the effect of external foreground extinction is not expected to be large at these long wavelengths, even with elevated levels of material along the LOS (see above). Indeed, we have also shown that the PBRs require a central heating source, indicating that the detection of a $70 \mu \mathrm{m}$ point source drives the interpretation of the sample as Class 0 sources, irrespective of source inclination. However, we note that if the central masses are significantly different than the assumed value of $0.5 M_{\odot}$, then for a fixed reference envelope density the inferred envelope infall rates need to be scaled accordingly (see Equation (1)).

To further investigate the evolutionary state of the PBRs, in Figure 14 we show the values of $L_{\mathrm{bol}}, T_{\mathrm{bol}}$, and $L_{\mathrm{smm}} / L_{\mathrm{bol}}$ for the entire sample of new candidate protostars and the previously identified Spitzer HOPS sample (W. J. Fischer et al., in preparation). In the left panel, we show $L_{\text {bol }}$ versus $T_{\text {bol }}$ for the entire sample of new protostar candidates, including those flagged as possible extragalactic contamination. We also show the four reference Class 0 sources presented in Table 8. The PBRs' sample, in particular, and the entire sample of new 

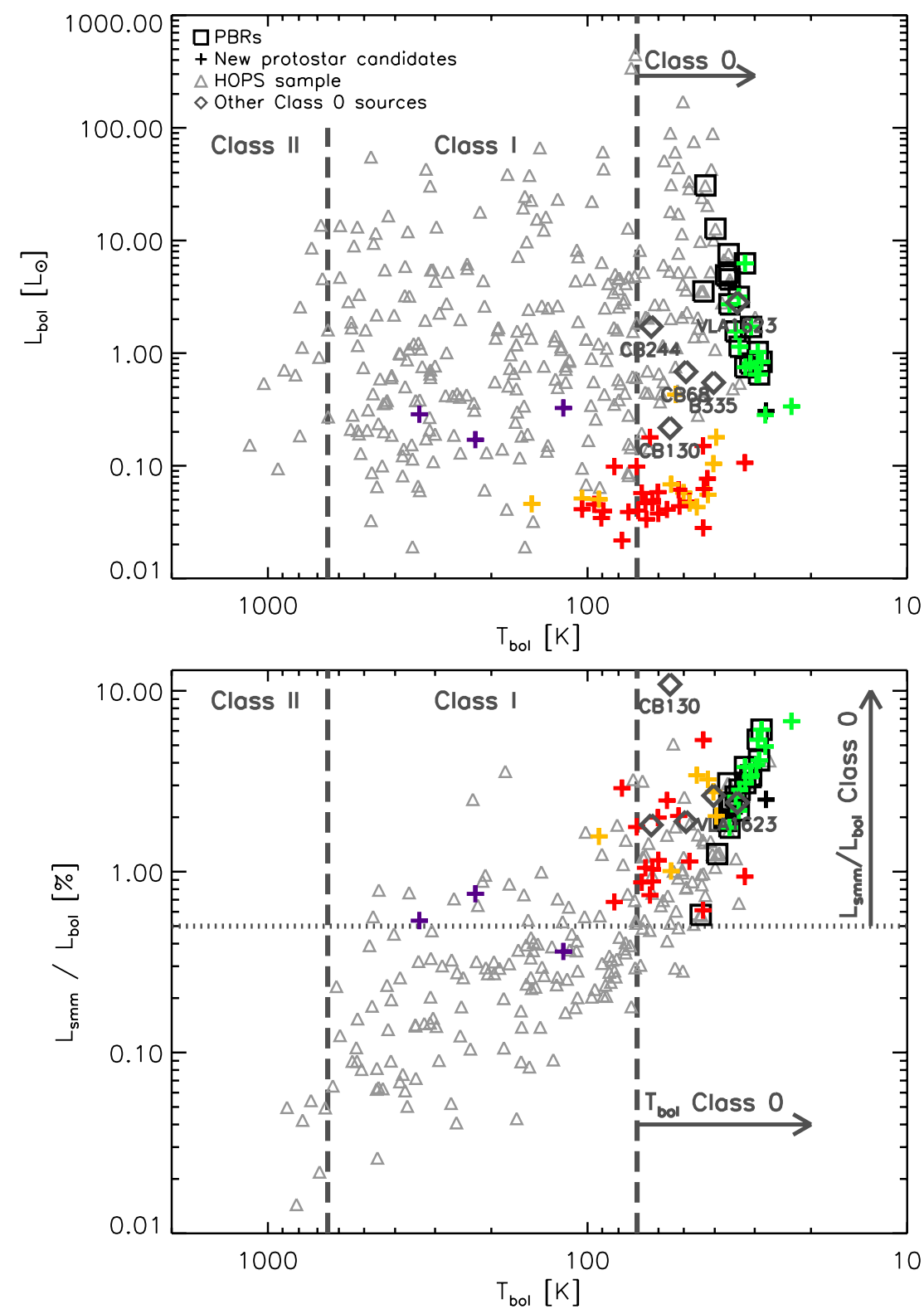

Figure 14. Top: bolometric luminosity vs. bolometric temperature for the new candidate protostars ( + symbols) and the HOPS protostars (light gray triangles). The color of the + symbols indicates the flag values shown in Table 3 and discussed in Section 3.2. The squares indicate the PBRs sample, drawn from both the new candidate protostar sample and the HOPS protostar sample. The dashed lines indicate the canonical $T_{\mathrm{bol}}$ divisions between protostellar classes. Bottom: $L_{\mathrm{smm}} / L_{\mathrm{bol}}$ vs. bolometric temperature for the subset of sources shown in the top panel for which we have sufficient sub-millimeter coverage to estimate $L_{\mathrm{smm}}$ (see the text). The horizontal dotted line indicates the André et al. (2000) proposed $L_{\mathrm{smm}} / L_{\mathrm{bol}}>0.5 \%$ Class 0 threshold.

(A color version of this figure is available in the online journal.)

candidate protostars are generally clustered around low $T_{\text {bol }}$ values. Ignoring inclination degeneracies and other considerations, these low $T_{\text {bol }}$ values indicate that the PBR sample is indeed composed of young Class 0 sources. In the right panel, we show $T_{\text {bol }}$ versus $L_{\mathrm{smm}} / L_{\text {bol }}$ for the sources with sufficient coverage to estimate $L_{\mathrm{smm}}$ (see Section 6.2). The PBRs, as expected if the sample can be explained as Class 0 sources, cluster around larger values of $L_{\mathrm{smm}} / L_{\mathrm{bol}}$ compared to the rest of the sample. André et al. (2000) proposed the $L_{\mathrm{smm}} / L_{\text {bol }}>0.5 \%$ threshold for Class 0 sources, and all but one of the new candidate protostars for which we can estimate $L_{\text {smm }}$ fall into this category. Irrespective of the evolutionary indicator that is chosen $\left(T_{\mathrm{bol}}\right.$ or $\left.L_{\mathrm{smm}} / L_{\mathrm{bol}}\right)$, all of the new candidate protostars in both the reliable and lower probability categories (green and yellow points, respectively), would be considered to be of Class 0 status. Finally, while the PBRs 70/24 > 1.65 color criterion causes some sources with very low values of $T_{\text {bol }}$ and very high values of $L_{\mathrm{smm}} / L_{\text {bol }}$ to be missed, the color selection is able to capture the vast majority of the most extreme Class 0 sources at the extrema of the $L_{\mathrm{smm}} / L_{\mathrm{bol}}$ and $T_{\mathrm{bol}}$ distributions.

This evidence strongly supports the interpretation of the PBRs (and indeed all the sources classified as reliable protostellar candidates) as very dense Class 0 protostars, irrespective of the source inclination. On the other hand, the new candidate protostar sample, taken as a whole, may be explained by a combination of the effects described above: high inclination, high densities, and extreme values of foreground extinction, along with elevated levels of extragalactic contamination. Of 
particular interest is the possibility that some of the sources classified as low probability protostars at low $L_{\text {bol }}$ values may be confirmed as bona fide protostars with future observations (see Offner \& McKee 2011 for a detailed discussion of the significance of such sources); this will be investigated in future work (A. M. Stutz et al., in preparation).

To definitely measure the inclinations of our sample of sources and to therefore determine envelope densities more accurately we require millimeter line emission maps at high resolution from, e.g., ALMA, along with single dish observations of high density tracers. A vigorous follow-up campaign is therefore underway to more firmly place these protostars within the context of star formation in the Orion clouds. We are observing ammonia spectra toward the full sample of protostar candidates to verify the presence of dense molecular gas and determine kinetic temperatures. A Herschel PACS range spectroscopy program toward eight PBRs will characterize the energetics of outflows and UV heating on small scales. The outflows (CO), dust continuum, and surrounding dense molecular gas $\left(\mathrm{N}_{2} \mathrm{H}^{+}\right)$are being observed in the millimeter to determine the source inclinations, outflow opening angles, inner envelope properties, and the kinematics of the larger scale dense gas.

\section{CONCLUSIONS}

We have discovered a sample of 55 new candidate protostars in Orion with Herschel, as part of the HOPS OTKP scan-map observations at $70 \mu \mathrm{m}$ and $160 \mu \mathrm{m}$. We conclude the following.

1. The new candidate protostars are either very faint or undetected at Spitzer wavelengths. We find 34 sources with $24 \mu \mathrm{m}$ magnitudes that are greater than 7.0 and 21 sources that are undetected in the MIPS $24 \mu \mathrm{m}$ band.

2. We analyze the IRAC colors and the broad shape of the SEDs between $3.6 \mu \mathrm{m}$ and $160 \mu \mathrm{m}$. Based on this analysis, we classify the sample as follows: $27 \%$ (15 sources) are considered reliable protostars, $18 \%$ (10 sources) are considered lower probability protostars, $47 \%$ (26 sources) are classified as extragalactic contamination, including AGNs, 3 sources have IRAC colors consistent with stellar photospheres but Herschel and APEX SEDs consistent with cold dust emission, and 1 source does not have IRAC coverage. We find that the subset of sources without $24 \mu \mathrm{m}$ detections dominates the number of sources categorized as most reliable protostellar candidates by a factor of $\sim 3$, suggesting that sources with no short-wavelength detections and only PACS $70 \mu \mathrm{m}$ and longer wavelength detections are much less likely to be of extragalactic origin.

3. We combine the new protostar candidate sample with the previously identified Spitzer HOPS sample and find that 18 sources have 70/24 colors greater than 1.65 . These are the reddest protostars known in Orion, 11 of which are newly identified Herschel sources in the reliable protostar category listed above. We name these sources PBRs. Compared to the other protostars in the HOPS fields, the PBRs populate the extrema in the distributions of standard evolutionary diagnostics, having both the largest $L_{\mathrm{smm}} / L_{\mathrm{bol}}$ ratios and lowest $T_{\text {bol }}$ values. The PBR source SEDs and peak SED wavelengths are consistent with the hypothesis that the PBRs do indeed represent a population composed of Class 0 sources with the densest envelopes in Orion.

4. A comparison to radiative transfer models of rotating, collapsing protostellar envelopes with outflow cavities show that the 70/24 >1.65 color limit selects sources with envelope densities with $\log \rho_{1} /\left(\mathrm{g} \mathrm{cm}^{-3}\right) \gtrsim-13.4$, irrespective of inclination effects. While the $70 / 24>1.65$ color selects sources with dense envelopes, this color criterion does not find all dense sources above the threshold of $\rho_{1} /\left(\mathrm{g} \mathrm{cm}^{-3}\right)=-13.4$. Therefore this selection should be used in conjunction with other evolutionary indicators, namely millimeter and sub-millimeter measurements, to determine the nature of the observed sources.

5. Our modeling of the PBRs' SEDs reveals that these sources are not consistent with being externally heated starless cores; the presence of a $70 \mu \mathrm{m}$ point source requires that the sample be interpreted as dense envelopes containing embedded protostars.

6. The fraction of known protostars that are PBRs varies from $1 \%$ in the Orion A cloud to $17 \%$ in the Orion B cloud, with an average fraction over the Orion complex of $5 \%$. These numbers suggest that if the PBRs represent a distinct phase in protostellar evolution, protostars spend on average $5 \%$ of their lifetime in the PBRs phase. Most of the PBRs in the Orion B cloud are concentrated in dense gas near the NGC 2068, NGC 2071, and NGC 2023 nebulae. These regions of dense gas are also known for a lack of more evolved pre-main-sequence stars. The high percentage of PBRs in Orion B suggests either that the regions containing the PBRs may currently be undergoing more vigorous star formation than other regions of Orion, or that the PBRs lifetime is longer in these regions.

7. The sources with $70 / 24<1.65$ colors and faint $70 \mu \mathrm{m}$ fluxes must be confirmed as either protostellar or contamination sources before their significance can be assessed. If confirmed as the former, however, this sample would constitute an important population of very low luminosity, cold protostars previously unobserved, most interesting from the point of view of constraining the faint end of the luminosity function of protostars.

8. We expect that a comparable number of very red protostars will be found with Herschel in more nearby starforming regions. We caution that a careful treatment of possible extragalactic contamination must be implemented to understand the broader significance of such sources.

The authors gratefully acknowledge help from Hélène Roussel in the production of Scanamorphos PACS maps. Furthermore, we are grateful to Oskari Miettinen for sharing the reduced SABOCA map of the 090003 region and to Joel Green for providing the SED VLA1623-243. A.M.S. kindly acknowledges helpful and insightful discussions with Ralf Launhardt. The work of A.M.S. was supported by the Deutsche Forschungsgemeinschaft priority program 1573 ("Physics of the Interstellar Medium"). J.T. acknowledges support provided by NASA through Hubble Fellowship grant \#HST-HF-51300.01A awarded by the Space Telescope Science Institute, which is operated by the Association of Universities for Research in Astronomy, Inc., for NASA, under contract NAS 5-26555. The National Radio Astronomy Observatory is a facility of the National Science Foundation operated under cooperative agreement by Associated Universities, Inc. This publication is based on data acquired with the Atacama Pathfinder Experiment (APEX). APEX is a collaboration between the Max-Planck-Institut für Radioastronomie, the European Southern Observatory, and the Onsala Space Observatory. The Herschel spacecraft was 
designed, built, tested, and launched under a contract to ESA managed by the Herschel/Planck Project team by an industrial consortium under the overall responsibility of the prime contractor Thales Alenia Space (Cannes), and including Astrium (Friedrichshafen), responsible for the payload module and for system testing at spacecraft level; Thales Alenia Space (Turin), responsible for the service module; and Astrium (Toulouse), responsible for the telescope, with in excess of a hundred subcontractors. PACS has been developed by a consortium of institutes led by MPE (Germany) and including UVIE (Austria); KU Leuven, CSL, IMEC (Belgium); CEA, LAM (France); MPIA (Germany); INAF-IFSI/OAA/OAP/OAT, LENS, SISSA (Italy); IAC (Spain). This development has been supported by the funding agencies BMVIT (Austria), ESA-PRODEX (Belgium), CEA/CNES (France), DLR (Germany), ASI/INAF (Italy), and CICYT/MCYT (Spain). HCSS/HSpot/HIPE is a joint development (are joint developments) by the Herschel Science Ground Segment Consortium, consisting of ESA, the NASA Herschel Science Center, and the HIFI, PACS, and SPIRE consortia. We also use the Spitzer Space Telescope and the Infrared Processing and Analysis Center (IPAC) Infrared Science Archive, which are operated by JPL/Caltech under a contract with NASA. This research has made use of the SIMBAD database and VizieR catalog access tool, operated at CDS, Strasbourg, France. Support for this work was provided by the National Aeronautics and Space Administration (NASA) through awards issued by the Jet Propulsion Laboratory, California Institute of Technology (JPL/Caltech).

\section{APPENDIX A}

\section{PREVIOUS PBRs DETECTIONS}

We searched the SIMBAD and VizieR (Ochsenbein et al. 2000) services for previous identifications of the PBRs. We restricted our search to a radius of $20^{\prime \prime}$ from the $70 \mu \mathrm{m}$ source coordinates. The results are summarized in Table 6 . This list is likely incomplete and is intended to provide a resource and rough guide to some of the previous detections of these sources.

061012, 119019, and 097002. None found.

HOPS169. Known protostar and outflow bipolar outflow V380 Ori NE (e.g., Davis et al. 2000). Stanke et al. (2002) detected source 59 in their $2.12 \mu \mathrm{m}$ catalog of Orion A, offset by 6 " from our source coordinates. Davis et al. (2009) also detected an outflow about $2^{\prime \prime}$ away from our coordinates. Nutter \& Ward-Thompson (2007) classified this source as hosting a YSO and measured an $850 \mu \mathrm{m}$ flux-derived mass of $2.8 \mathrm{M}_{\odot}$ (assuming a temperature of $20 \mathrm{~K}$ and a distance of $400 \mathrm{pc}$ ).

019003. Since this source is located in the very crowded and complex ONC filament, it is often not clear which previous identifications may be associated with it in particular. Tsujimoto et al. (2003) listed a near-IR source located 3".35 away from our coordinates. Nutter \& Ward-Thompson (2007) detected a source offset by 8 ."4; they classified it as hosting a YSO and measure a mass of $6.2 M_{\odot}$. While it is not clear if the detection is associated with the PBRs, we consider it probable. Chini et al. (1997) and Nielbock et al. (2003) likely detected this source in their $1.3 \mathrm{~mm}$ maps near FIR1a in OMC-2, although their beam sizes were too large to identify the source unambiguously.

082005. This source was previously classified as a starless core (e.g., Johnstone \& Bally 2006; Nutter \& Ward-Thompson
2007; Mookerjea et al. 2009). The Nutter \& Ward-Thompson (2007) location of the source is about $8^{\prime \prime}$ away from our source with an associated mass of $3.3 M_{\odot}$. Mookerjea et al. (2009) measured a mass of $4.3 M_{\odot}$ for this source.

HOPS372 and 082012. These sources have coordinates that are significantly offset from the Nutter \& Ward-Thompson (2007) coordinates; the two sources are unresolved and lie about $13^{\prime \prime}$ away from their catalog entry, with a mass of 12.7 $M_{\odot}$. The Mookerjea et al. (2009) analysis derived a mass of $1 M_{\odot}$ for $082012(\mathrm{MM} 1)$ and $7.4 M_{\odot}$ for HOPS372 (MM2), from modified blackbody fits to long-wavelength SEDs.

090003. Miettinen et al. (2009) detected this source (SMM3) in their $850 \mu \mathrm{m}$ map, and concluded that it is a promising Class 0 candidate based on the shape of the SED. They calculated a mass of about 7.5 $M_{\odot}$ for this source. Miettinen et al. (2010, 2012) also observed this source in various molecular line transitions and with SABOCA at $350 \mu \mathrm{m}$; their measured flux for this source is $S_{350}^{\text {peak }}=3.63 \mathrm{Jy} \mathrm{beam}^{-1}$. We include their flux measurement and the $350 \mu \mathrm{m}$ map in our analysis.

HOPS358. Strom et al. (1976, 1986), and (Reipurth et al. 1999) detected the Herbig-Haro complex HH24-26, with $\mathrm{HH} 25$ located about $10^{\prime \prime}$ away from HOPS358. This source is also included in the Wu et al. (2004) high velocity outflow catalog. Nutter \& Ward-Thompson (2007) classified a nearby source ( $11^{\prime \prime}$ away) as a starless core with a mass of $6.3 M_{\odot}$.

091015 and 091016. These sources were detected by, e.g., Lis et al. (1999) at $1.3 \mathrm{~mm}$ and $350 \mu \mathrm{m}$ (as sources 5 and 6 ). The reported masses are $\sim 2 M_{\odot}$ for each source. Both sources are classified as starless by Nutter \& Ward-Thompson (2007), with catalog masses of $0.8 M_{\odot}(091015)$ and $1.3 M_{\odot}$ (091016).

HOPS373 and 093005. Both sources were classified as starless by Nutter \& Ward-Thompson (2007), with reported masses of $4.2 M_{\odot}$ (HOPS373) and 3.9 $M_{\odot}$ (093005). Motte et al. (2001) observed this region at 450 and $850 \mu \mathrm{m}$ and also classified 093005 as a starless core, based on the lack of evidence for an embedded source. Source HOPS373, on the other hand, is known to be driving a CO outflow (Gibb \& Little 2000), and therefore has been classified as a candidate Class 0 source. Furthermore, Haschick et al. (1983) identified a water maser near the HOPS373 location.

302002. Nutter \& Ward-Thompson (2007) reported a mass of $2.7 M_{\odot}$ and classified this source as protostellar. Phillips et al. (2001) classify this source (LBS18S) as pre-protostellar, however.

HOPS359. Nutter \& Ward-Thompson (2007) classified this source as protostellar and measure a mass of $2.7 \mathrm{M}_{\odot}$.

HOPS341. This source is strongly blended with HOPS340. We have listed some detections of the combined system in Table 6.

HOPS354. Reipurth et al. (2008) described this source in the context of the L1622 cloud. Bally et al. (2009) also detected this source in their analysis of Spitzer IRAC images but did not analyze it in detail.

\section{APPENDIX B}

\section{GALLERY OF IMAGES OF PACS BRIGHT RED SOURCES}

Here we present the gallery of images of the remaining PBRs, shown in Figures 15-25. 

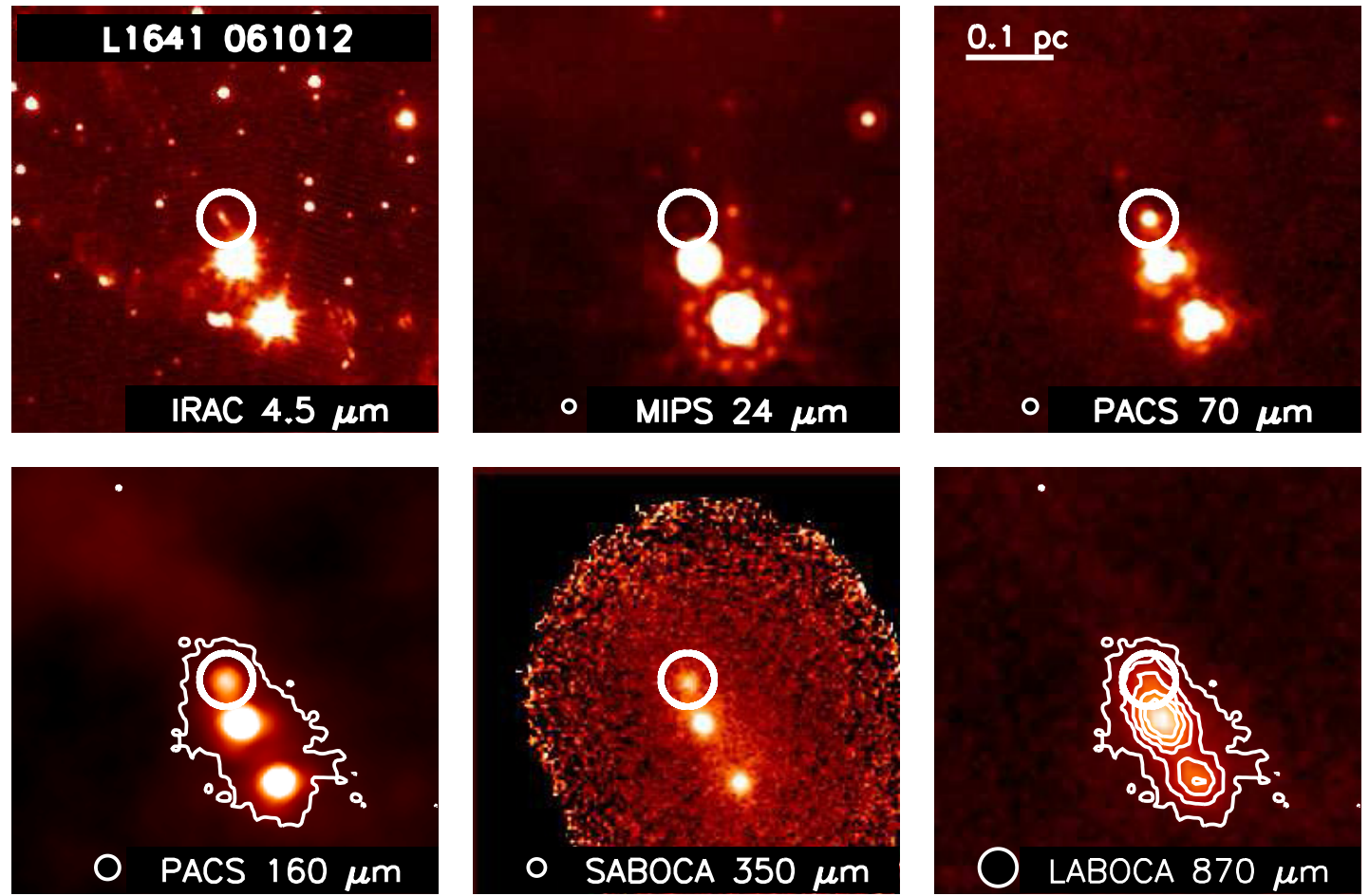

Figure 15. Same as Figure 7, showing $4^{\prime} \times 4^{\prime}$ images of PBR 061012. The IRAC-band emission associated with the source is clearly visible at $4.5 \mu \mathrm{m}$. Contours indicate the $870 \mu \mathrm{m}$ emission levels at $\{0.1,0.25,0.4,0.55,0.7\} \mathrm{Jy}_{\text {beam }}^{-1}$; the long wavelength sub-millimeter data trace the cold envelope material associated with the source. The $160 \mu \mathrm{m}$ panel is shown with the lowest $870 \mu \mathrm{m}$ emission contour.

(A color version of this figure is available in the online journal.)
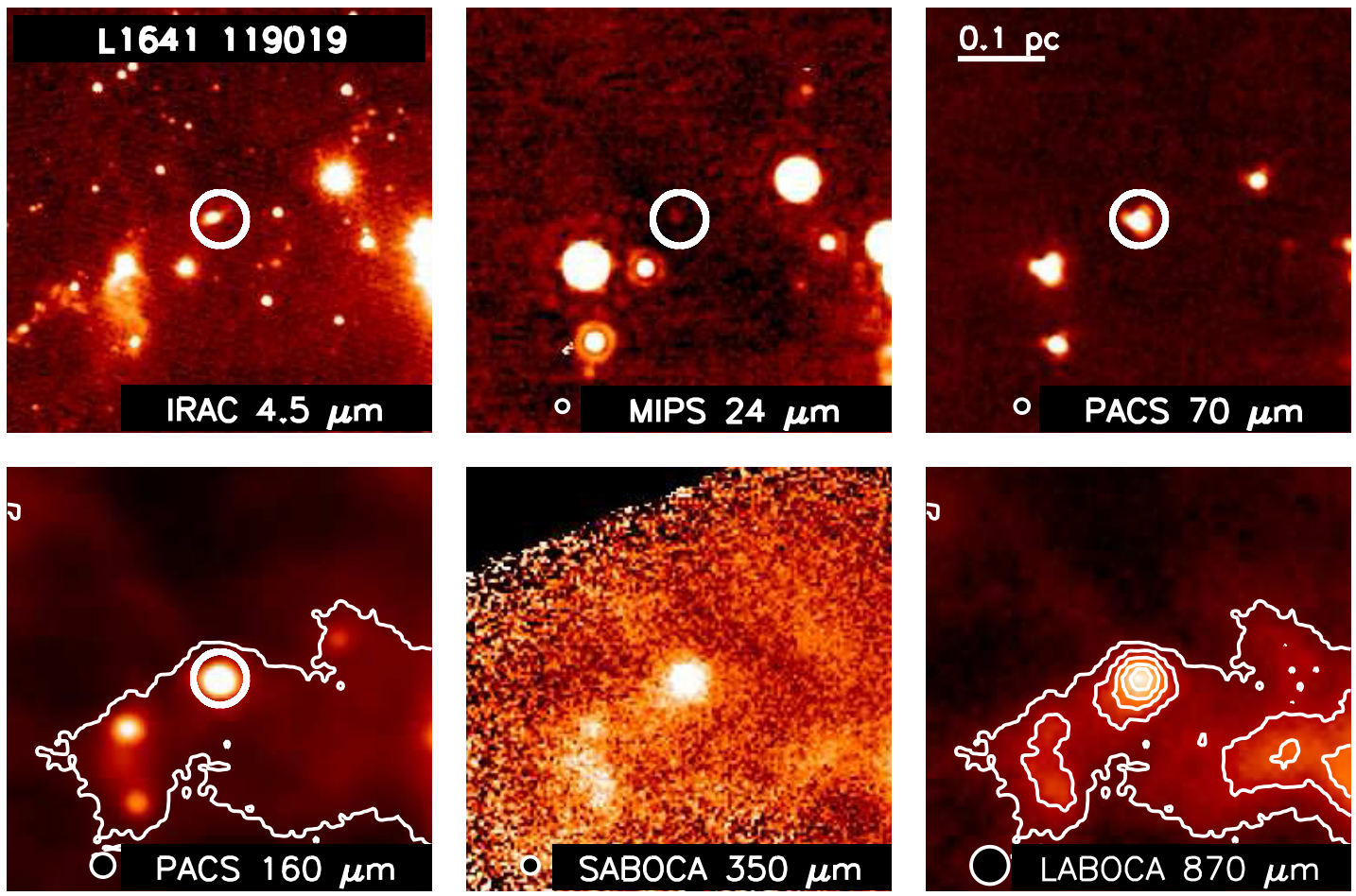

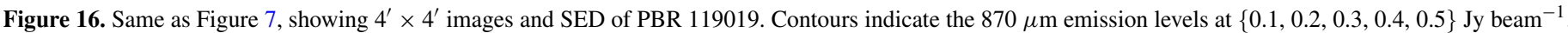
This source has prominent IRAC emission and is located in an IRAC $8 \mu \mathrm{m}$ and MIPS $24 \mu \mathrm{m}$ shadow.

(A color version of this figure is available in the online journal.) 

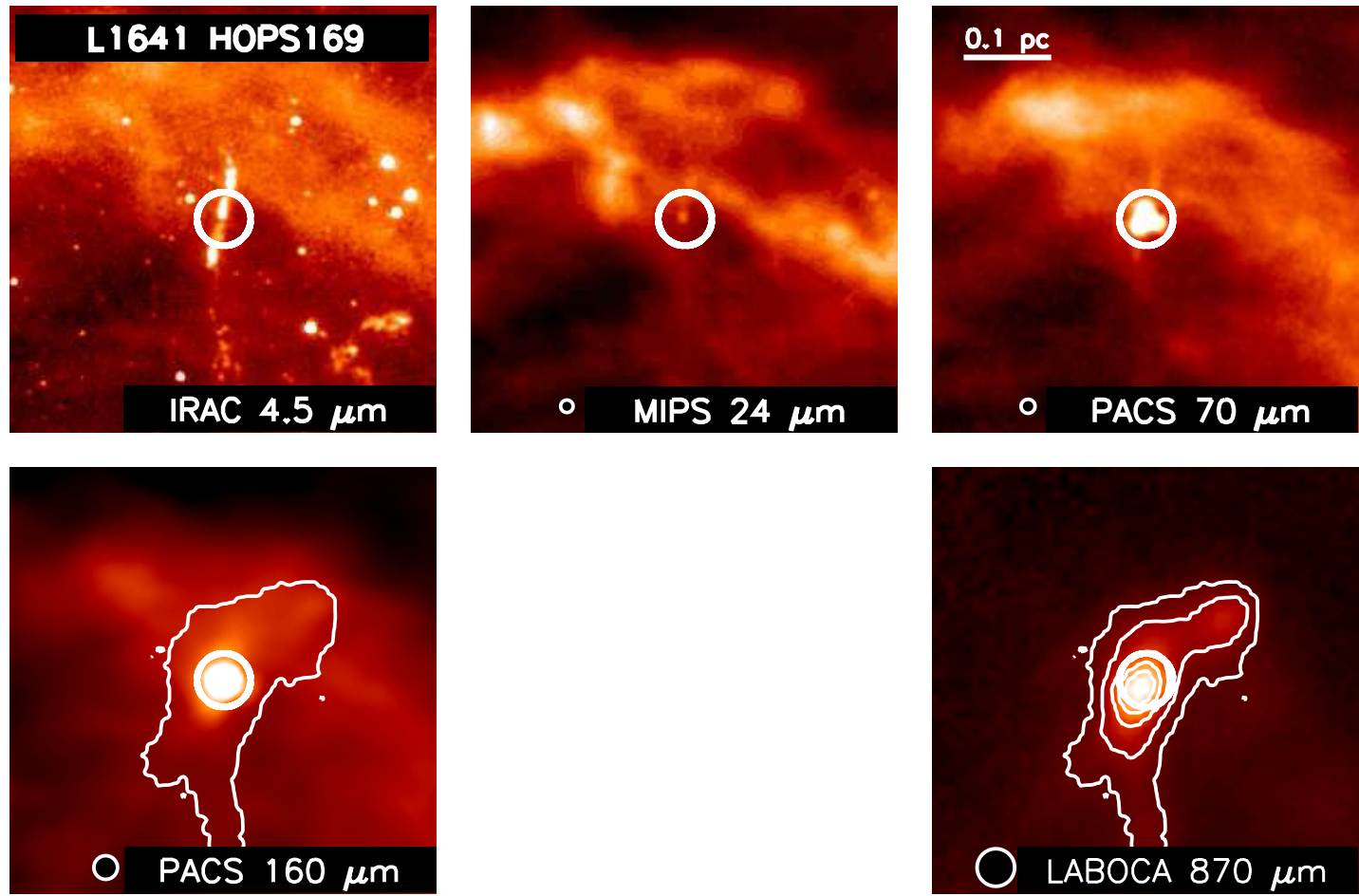

Figure 17. Same as Figure 7, showing $4^{\prime} \times 4^{\prime}$ images and SED of HOPS169. Contours indicate the $870 \mu \mathrm{m}$ emission levels at $\{0.1,0.2,0.4,0.6,0.8\} \mathrm{Jy}^{-1}$ beam ${ }^{-1}$ This source has clear outflow activity, traced by the IRAC emission, and appears to be at high inclination.

(A color version of this figure is available in the online journal.)
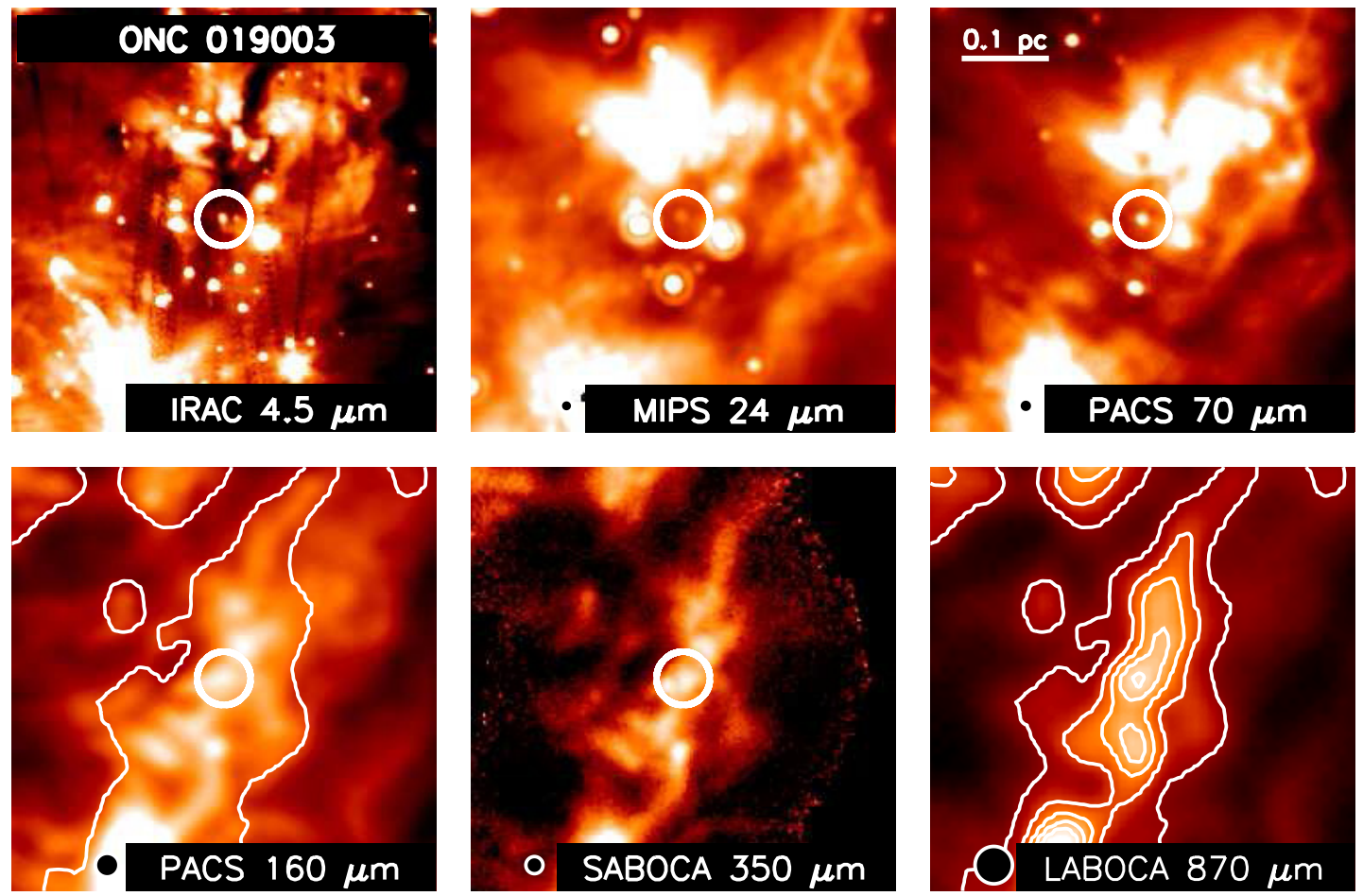

Figure 18. Same as Figure 7, showing $4^{\prime} \times 4^{\prime}$ images and SED of PBR source 019003. Contours indicate the $870 \mu \mathrm{m}$ emission levels at $\{0.5,1.0,1.5,2.0,2.5\}$ $\mathrm{Jy} \mathrm{beam}^{-1}$. This source has indications of outflow activity, as traced by the IRAC emission. The Herschel photometry may be strongly affected by blending due to the source location in a very dense filament.

(A color version of this figure is available in the online journal.) 

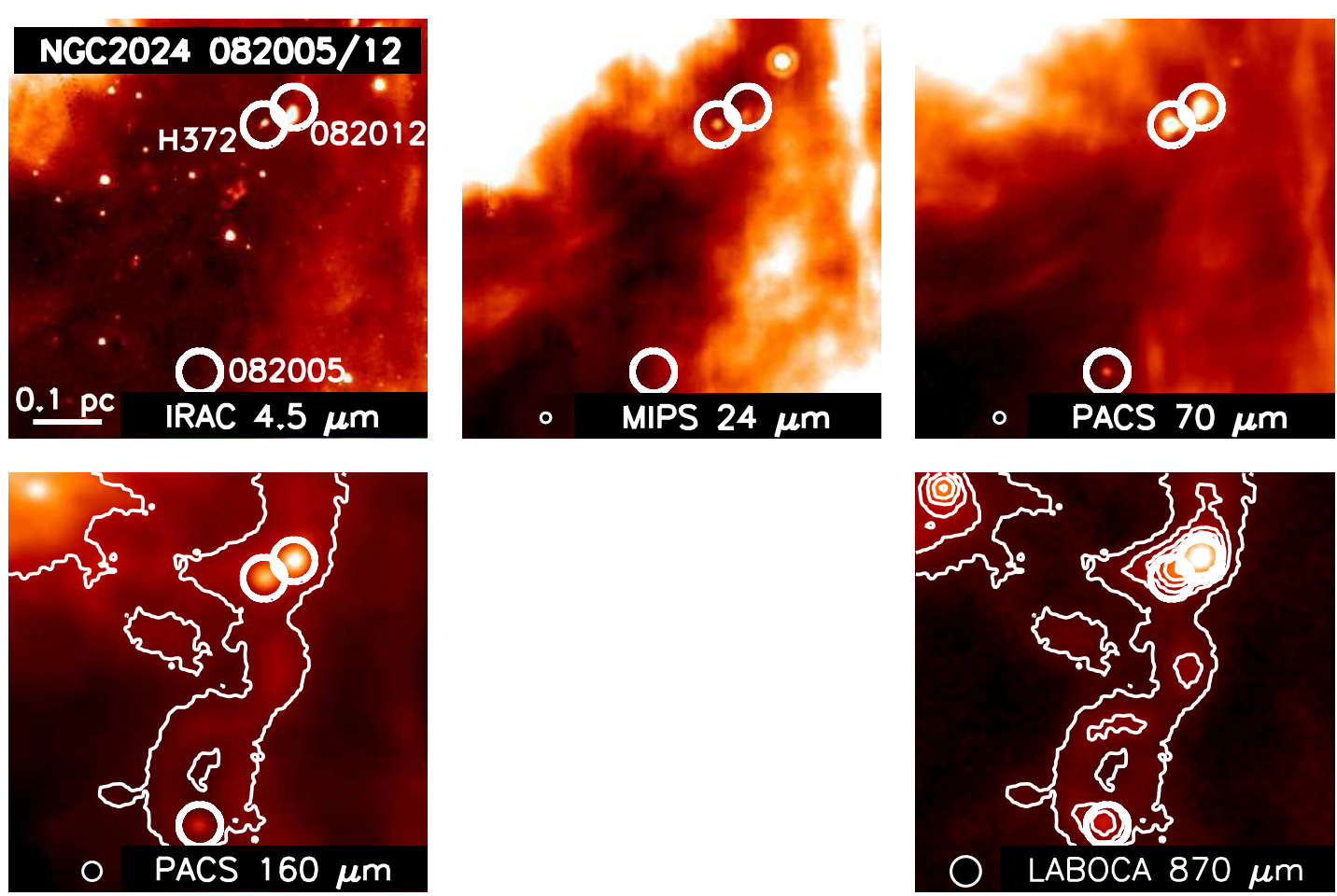

Figure 19. Top: same as Figure 7, showing $5^{\prime} \times 5^{\prime}$ images of three red sources: HOPS372 and 082012 (top) and 082005 (bottom). Contours indicate the $870 \mu \mathrm{m}$ emission levels at $\{0.25,0.5,0.75,1.0,1.25,1.5\} \mathrm{Jy} \mathrm{beam}^{-1}$. No IRAC emission is detected for 082005 ; however, this source is located in dense filamentary material traced by the sub-millimeter emission and an $8 \mu \mathrm{m}$ absorption feature.

(A color version of this figure is available in the online journal.)
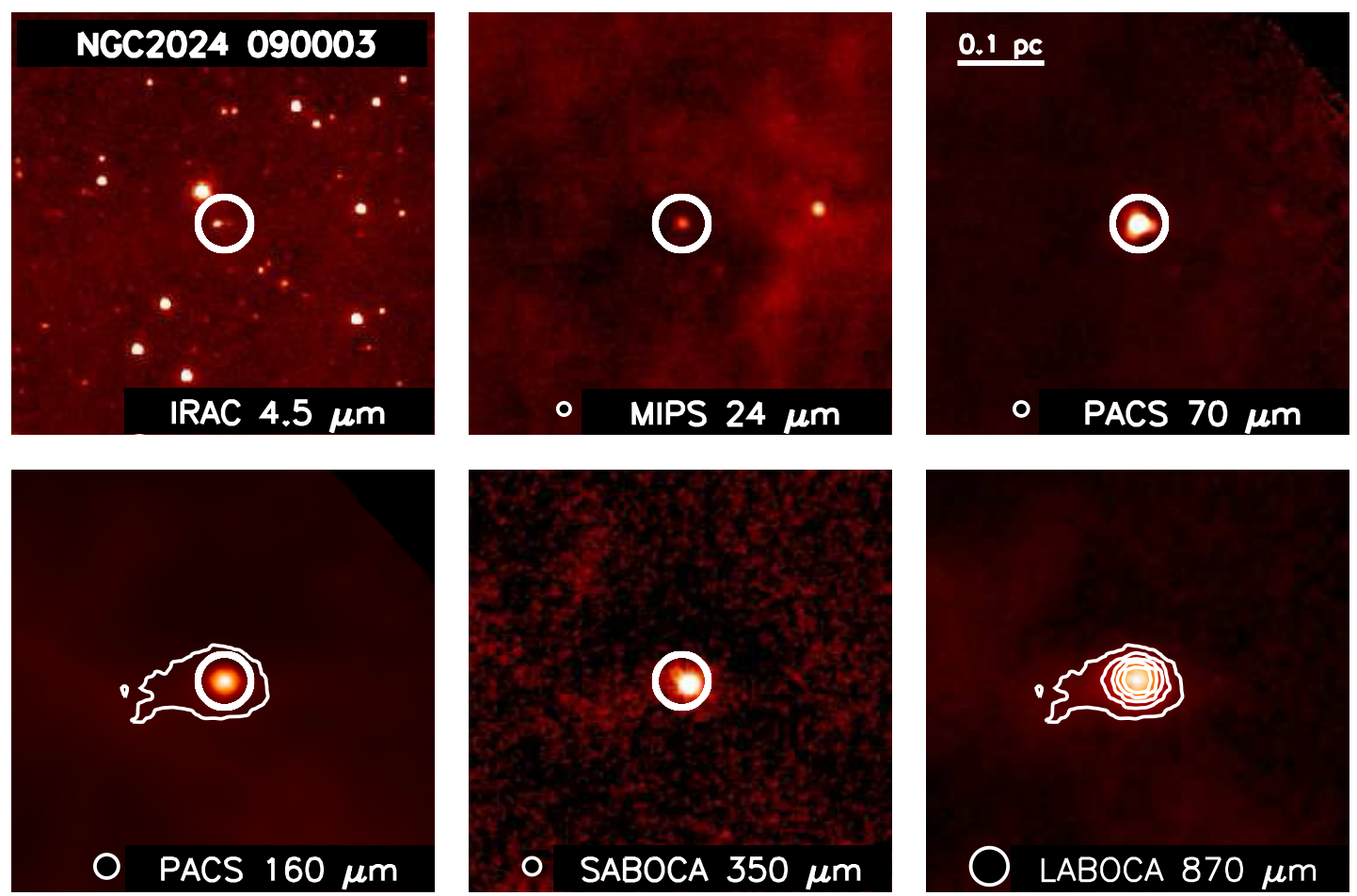

Figure 20. Same as Figure 7, showing $4^{\prime} \times 4^{\prime}$ images and SED of PBR 090003. Contours indicate the $870 \mu \mathrm{m}$ emission levels at $\{0.25,0.5,0.75,1.0,1.25,1.5\}$ $\mathrm{Jy} \mathrm{beam}^{-1}$. The IRAC data show faint indications of extended emission, possibly associated with an outflow activity or a second source. The $350 \mu \mathrm{m}$ image and SED point are from Miettinen et al. (2012).

(A color version of this figure is available in the online journal.) 

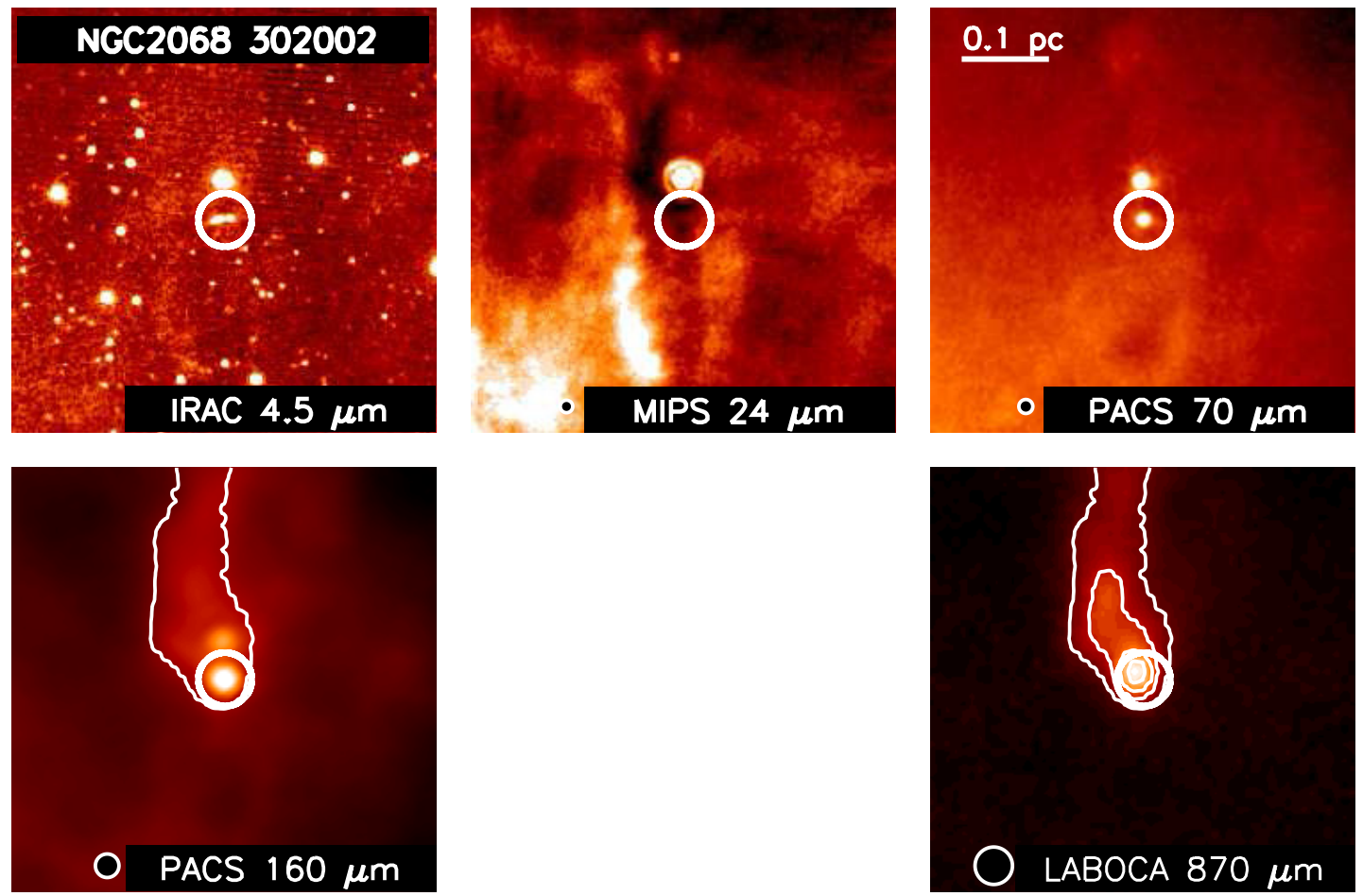

Figure 21. Same as Figure 7; $4^{\prime} \times 4^{\prime}$ images of PBR 302002. Contours indicate the $870 \mu \mathrm{m}_{\text {emission levels at }\{0.25,0.5,0.75,1.0,1.25,1.5\} \mathrm{Jy} \text { beam }}{ }^{-1}$, tracing the cometary-globule-shaped region. The IRAC data show indications of emission associated with outflow activity; furthermore, the $4.5 \mu \mathrm{m}$ data show evidence that this source is observed at high inclination. This source is the second most massive source in our sample, with a best-fit $M_{\text {env }}=1.7 M_{\odot}$.

(A color version of this figure is available in the online journal.)
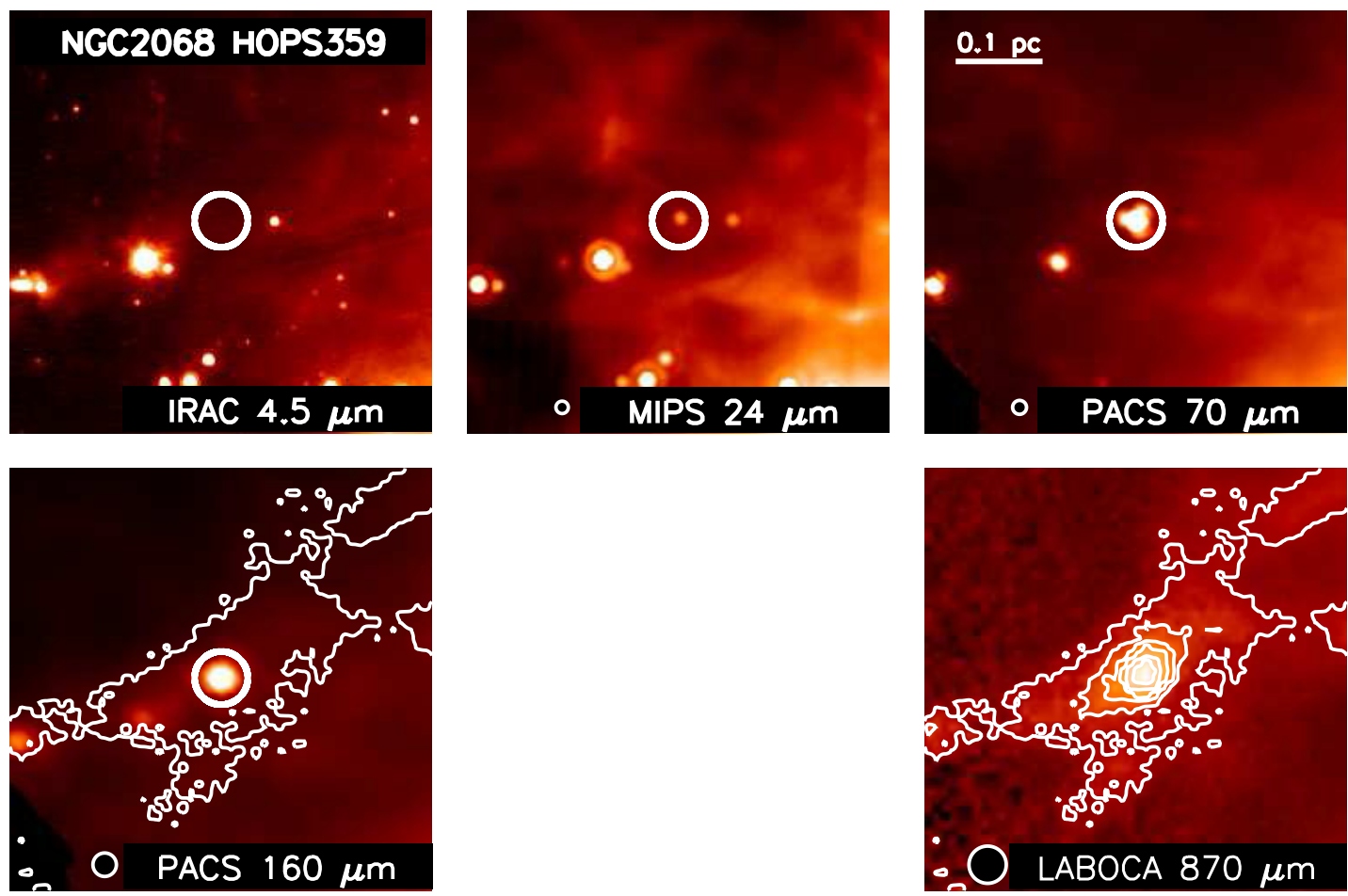

Figure 22. Same as Figure 7; $4^{\prime} \times 4^{\prime}$ images of HOPS359. Contours indicate the $870 \mu \mathrm{m}$ emission levels at $\{0.25,0.5,0.75,1.0,1.25,1.5\} \mathrm{Jy}^{\text {beam }}{ }^{-1}$. (A color version of this figure is available in the online journal.) 

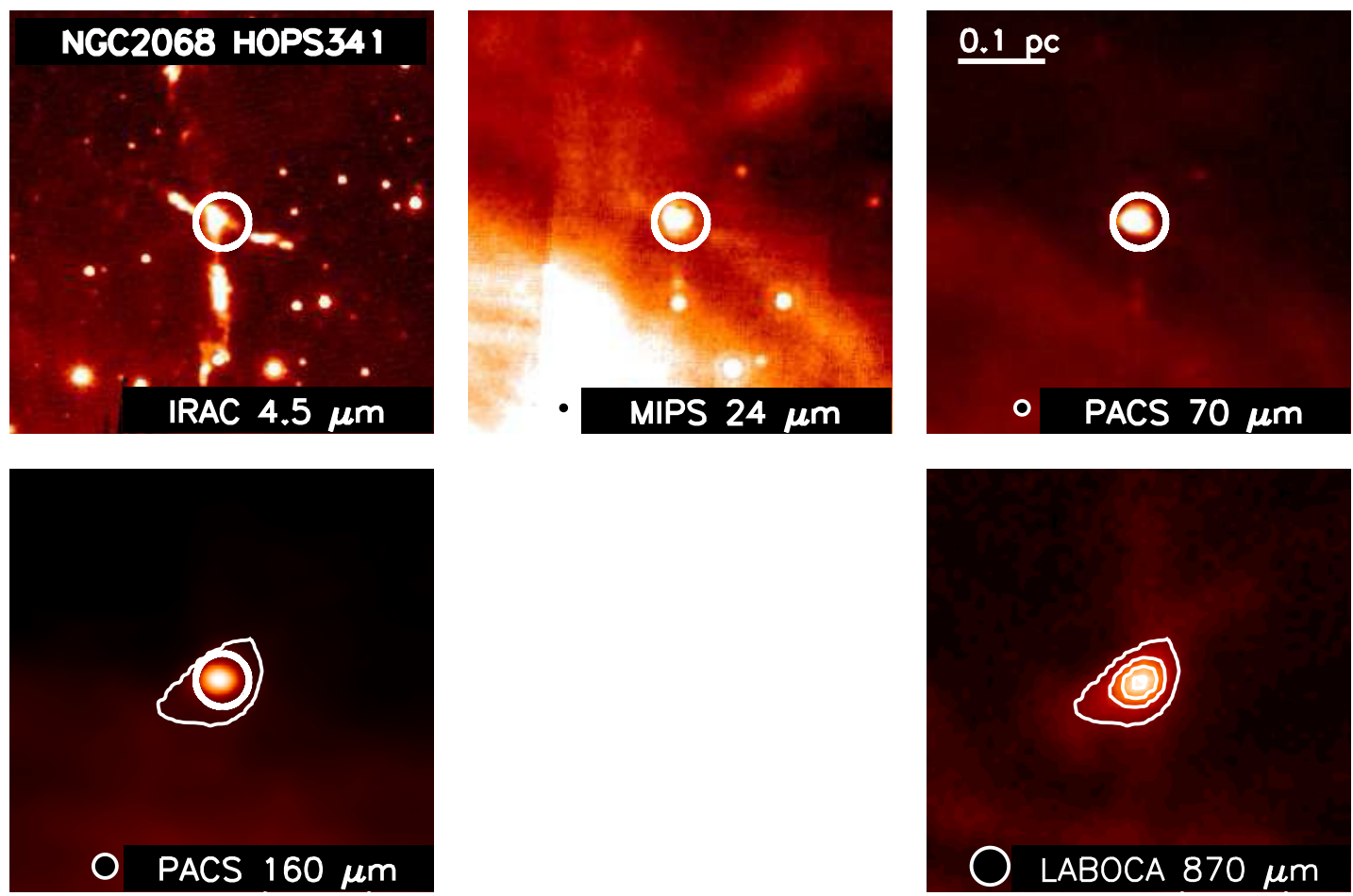

Figure 23. Same as Figure 7; $4^{\prime} \times 4^{\prime}$ images of HOPS341. Contours indicate the $870 \mu \mathrm{m}$ emission levels at $\{0.25,0.5,0.75,1.0\}$ Jy beam $^{-1}$. The $\times$-shaped morphology of this source in the IRAC bands indicated that it is a binary. Indeed, the photometry of this source is strongly blended with HOPS340; nevertheless, we include this source in our sample for completeness.

(A color version of this figure is available in the online journal.)
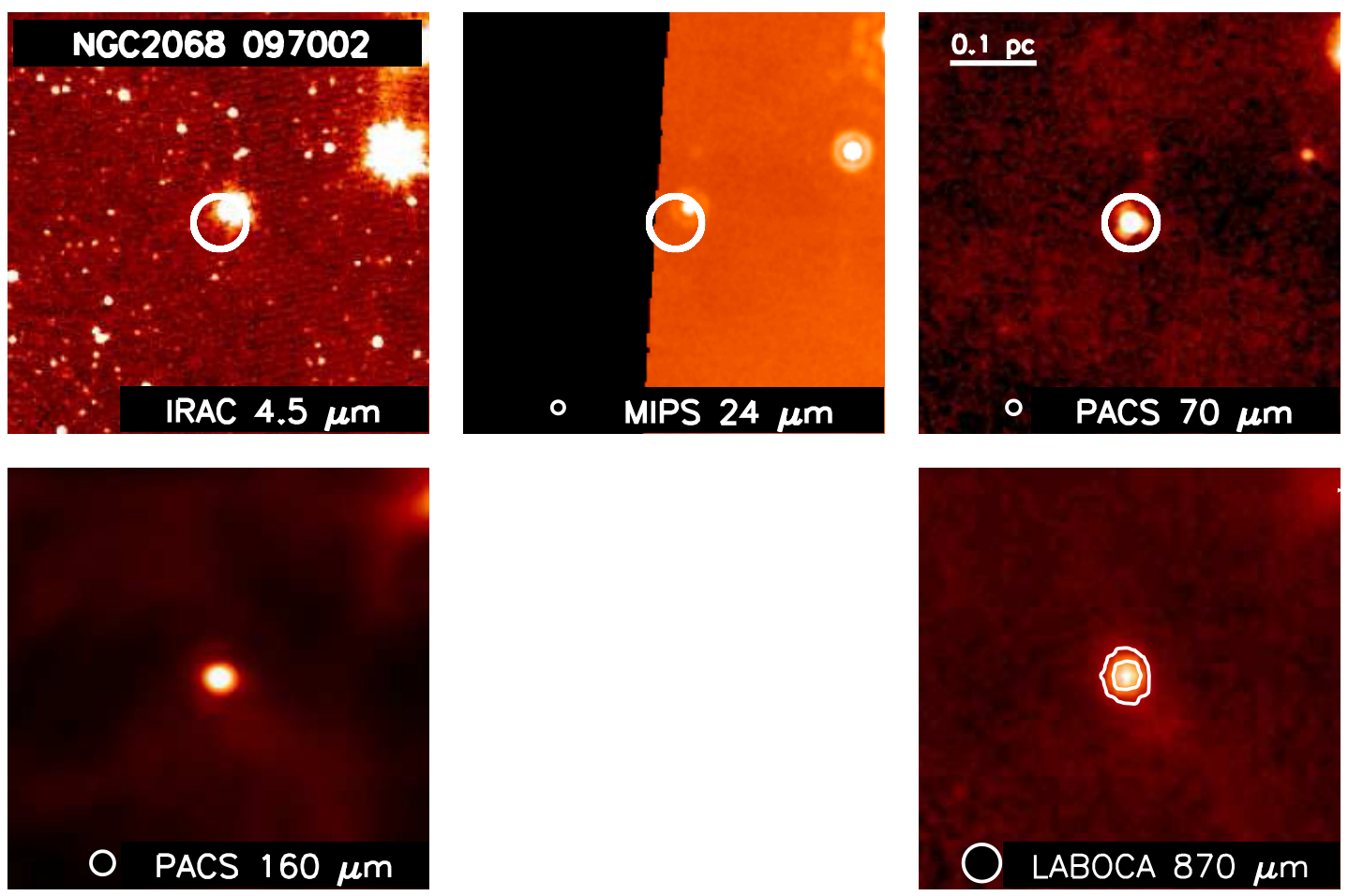

Figure 24. Same as Figure 7; $4^{\prime} \times 4^{\prime}$ images of PBR 097002. Contours indicate the $870 \mu \mathrm{m}$ emission levels at $\{0.25,0.5\} \mathrm{Jy} \mathrm{beam}^{-1}$. (A color version of this figure is available in the online journal.) 

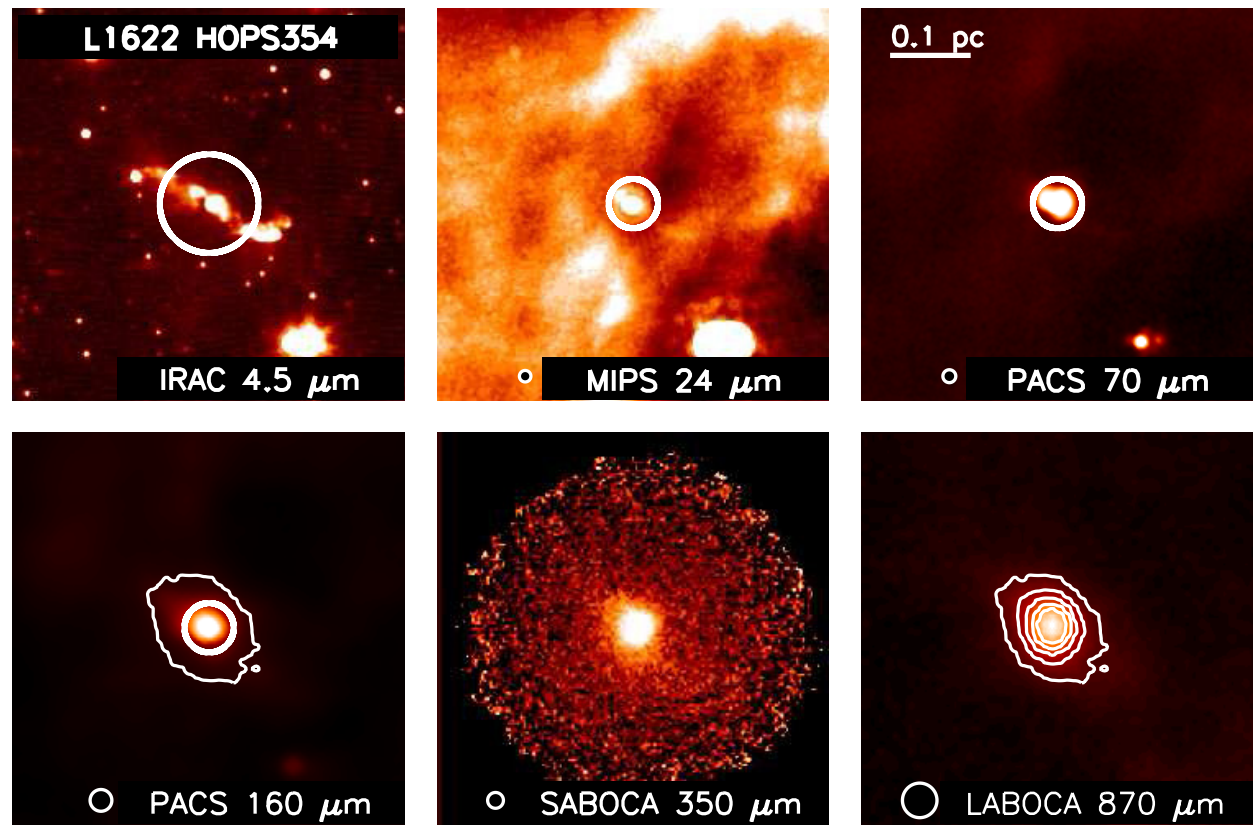

Figure 25. Same as Figure 7; $4^{\prime} \times 4^{\prime}$ images of HOPS354. Contours indicate the $870 \mu \mathrm{m}^{\text {emission levels at }\{0.25,0.5,0.75,1.0,1.25\} \mathrm{Jy}^{\mathrm{b}} \text { beam }}{ }^{-1}$. The IRAC images display clear indications of outflow activity; furthermore, the source appears highly inclined.

(A color version of this figure is available in the online journal.)

\section{REFERENCES}

Ali, B., Tobin, J. J., Fischer, W. J., et al. 2010, A\&A, 518, L119 Allen, L. E., Calvet, N., D’Alessio, P., et al. 2004, ApJS, 154, 363 André, P., Men'shchikov, A., Bontemps, S., et al. 2010, A\&A, 518, L102 André, P., Ward-Thompson, D., \& Barsony, M. 1993, ApJ, 406, 122

André, P., Ward-Thompson, D., \& Barsony, M. 2000, in Protostars and Planets IV, ed. V. Mannings, A. P. Boss, \& S. S. Russell (Tucson, AZ: Univ. Arizona Press), 59

Aniano, G., Draine, B. T., Gordon, K. D., \& Sandstrom, K. 2011, PASP, 123,1218

Arce, H. G., \& Sargent, A. I. 2006, ApJ, 646, 1070

Bally, J., Walawender, J., Reipurth, B., \& Megeath, S. T. 2009, AJ, 137, 3843

Bourke, T. L., Myers, P. C., Evans, N. J., II, et al. 2006, ApJL, 649, L37

Cassen, P., \& Moosman, A. 1981, Icar, 48, 353

Chen, X., Arce, H. G., Zhang, Q., et al. 2010, ApJ, 715, 1344

Chini, R., Reipurth, B., Ward-Thompson, D., et al. 1997, ApJL, 474, L135

Commerçon, B., Launhardt, R., Dullemond, C., \& Henning, T. 2012, A\&A, 545, A98

Da Rio, N., Robberto, M., Hillenbrand, L. A., Henning, T., \& Stassun, K. G. 2012, ApJ, 748, 14

Davis, C. J., Dent, W. R. F., Matthews, H. E., Coulson, I. M., \& McCaughrean, M. J. 2000, MNRAS, 318, 952

Davis, C. J., Froebrich, D., Stanke, T., et al. 2009, A\&A, 496, 153

De Buizer, J. M., Osorio, M., \& Calvet, N. 2005, ApJ, 635, 452

Di Francesco, J., Johnstone, D., Kirk, H., MacKenzie, T., \& Ledwosinska, E. 2008, ApJS, 175, 277

Draine, B. T., \& Lee, H. M. 1984, ApJ, 285, 89

Dunham, M. M., Crapsi, A., Evans, N. J., II, et al. 2008, ApJS, 179, 249

Dunham, M. M., Evans, N. J., II, Bourke, T. L., et al. 2006, ApJ, 651, 945

Dunham, M. M., Evans, N. J., II, Terebey, S., Dullemond, C. P., \& Young, C. H. 2010, ApJ, 710, 470

Enoch, M. L., Evans, N. J., II, Sargent, A. I., \& Glenn, J. 2009, ApJ, 692, 973

Enoch, M. L., Lee, J.-E., Harvey, P., Dunham, M. M., \& Schnee, S. 2010, ApJL, 722, L33

Evans, N. J., II, Dunham, M. M., Jørgensen, J. K., et al. 2009, ApJS, 181, 321

Fang, M., van Boekel, R., Wang, W., et al. 2009, A\&A, 504, 461

Fischer, W. J., Megeath, S. T., Ali, B., et al. 2010, A\&A, 518, L122

Fischer, W. J., Megeath, S. T., Tobin, J. J., et al. 2012, ApJ, 756, 99

Flaherty, K. M., \& Muzerolle, J. 2008, AJ, 135, 966

Flaherty, K. M., Pipher, J. L., Megeath, S. T., et al. 2007, ApJ, 663, 1069

Furlan, E., McClure, M., Calvet, N., et al. 2008, ApJS, 176, 184

Gibb, A. G., \& Little, L. T. 2000, MNRAS, 313, 663

Gordon, K. D., Rieke, G. H., Engelbracht, C. W., et al. 2005, PASP, 117, 503

Gutermuth, R. A., Megeath, S. T., Myers, P. C., et al. 2009, ApJS, 184, 18
Gutermuth, R. A., Myers, P. C., Megeath, S. T., et al. 2008, ApJ, 674, 336 Hartmann, L. 2009, Accretion Processes in Star Formation (2nd ed.; Cambridge: Cambridge Univ. Press)

Harvey, P., Merín, B., Huard, T. L., et al. 2007, ApJ, 663, 1149

Haschick, A. D., Moran, J. M., Rodriguez, L. F., \& Ho, P. T. P. 1983, ApJ, 265,281

Hatchell, J., Fuller, G. A., Richer, J. S., Harries, T. J., \& Ladd, E. F. 2007, A\&A, 468,1009

Hirota, T., Bushimata, T., Choi, Y. K., et al. 2007, PASJ, 59, 897

Hsu, W.-H., Hartmann, L., Allen, L., et al. 2012, ApJ, 752, 59

Johnston, K. G., Shepherd, D. S., Robitaille, T. P., \& Wood, K. 2012, A\&A, 551, A43

Johnstone, D., \& Bally, J. 2006, ApJ, 653, 383

Johnstone, D., Fich, M., Mitchell, G. F., \& Moriarty-Schieven, G. 2001, ApJ, 559,307

Jørgensen, J. K., Johnstone, D., Kirk, H., \& Myers, P. C. 2007, ApJ, 656, 293

Jørgensen, J. K., van Dishoeck, E. F., Visser, R., et al. 2009, A\&A, 507, 861

Kenyon, S. J., \& Hartmann, L. W. 1990, ApJ, 349, 197

Kenyon, S. J., Whitney, B. A., Gomez, M., \& Hartmann, L. 1993, ApJ, 414, 773

Kim, S.-H., Martin, P. G., \& Hendry, P. D. 1994, ApJ, 422, 164

Könyves, V., André, P., Men'shchikov, A., et al. 2010, A\&A, 518, L106

Kryukova, E., Megeath, S. T., Gutermuth, R. A., et al. 2012, AJ, 144, 31

Lada, C. J. 1987, in IAU Symp. 115, Star Forming Regions, ed. M. Peimbert \&

J. Jugaku (Cambridge: Cambridge Univ. Press), 1

Lada, E. A. 1992, ApJL, 393, L25

Lada, E. A., Bally, J., \& Stark, A. A. 1991a, ApJ, 368, 432

Lada, E. A., Depoy, D. L., Evans, N. J., II, \& Gatley, I. 1991b, ApJ, 368, 432

Lada, E. A., Evans, N. J., II, \& Falgarone, E. 1997, ApJ, 488, 286

Larson, R. B. 1969, MNRAS, 145, 271

Launhardt, R., Mezger, P. G., Haslam, C. G. T., et al. 1996, A\&A, 312, 569

Launhardt, R., Nutter, D., Ward-Thompson, D., et al. 2010, ApJS, 188, 139

Launhardt, R., Stutz, A., Schmiedeke, A., et al. 2013, A\&A, in press

Levreault, R. M. 1988, ApJS, 67, 283

Lis, D. C., Menten, K. M., \& Zylka, R. 1999, ApJ, 527, 856

Manoj, P., Watson, D. M., Neufeld, D. A., et al. 2013, ApJ, 763, 83

Mathis, J. S., Mezger, P. G., \& Panagia, N. 1983, A\&A, 128, 212

Megeath, S. T., Allen, L. E., Gutermuth, R. A., et al. 2004, ApJS, 154, 367

Megeath, S. T., et al. 2012, ApJ, in press

Men'shchikov, A., André, P., Didelon, P., et al. 2010, A\&A, 518, L103

Menten, K. M., Reid, M. J., Forbrich, J., \& Brunthaler, A. 2007, A\&A, 474, 515

Mezger, P. G., Zylka, R., \& Wink, J. E. 1990, A\&A, 228, 95

Miettinen, O., Harju, J., Haikala, L. K., \& Juvela, M. 2010, A\&A, 524, A91

Miettinen, O., Harju, J., Haikala, L. K., \& Juvela, M. 2012, A\&A, 538, A137

Miettinen, O., Harju, J., Haikala, L. K., Kainulainen, J., \& Johansson, L. E. B. 2009, A\&A, 500, 845 
Mitchell, G. F., Johnstone, D., Moriarty-Schieven, G., Fich, M., \& Tothill, N. F. H. 2001, ApJ, 556, 215

Mookerjea, B., Sandell, G., Jarrett, T. H., \& McMullin, J. P. 2009, A\&A, 507,1485

Morgan, J. A., Schloerb, F. P., Snell, R. L., \& Bally, J. 1991, ApJ, 376, 618

Motte, F., André, P., Ward-Thompson, D., \& Bontemps, S. 2001, A\&A, 372, L41

Myers, P. C., Adams, F. C., Chen, H., \& Schaff, E. 1998, ApJ, 492, 703

Myers, P. C., \& Ladd, E. F. 1993, ApJL, 413, L47

Nielbock, M., Chini, R., \& Müller, S. A. H. 2003, A\&A, 408, 245

Nielbock, M., Launhardt, R., Steinacker, J., et al. 2012, A\&A, 547, A11

Noriega-Crespo, A., Moro-Martin, A., Carey, S., et al. 2004, ApJS, 154, 402

Nutter, D., \& Ward-Thompson, D. 2007, MNRAS, 374, 1413

Ochsenbein, F., Bauer, P., \& Marcout, J. 2000, A\&AS, 143, 23

Offner, S. S. R., \& McKee, C. F. 2011, ApJ, 736, 53

Offner, S. S. R., Robitaille, T. P., Hansen, C. E., McKee, C. F., \& Klein, R. I. 2012, ApJ, 753, 98

Ormel, C. W., Min, M., Tielens, A. G. G. M., Dominik, C., \& Paszun, D. 2011, A\&A, 532, A43

Ossenkopf, V., \& Henning, T. 1994, A\&A, 291, 943

Padgett, D. L., Brandner, W., Stapelfeldt, K. R., et al. 1999, AJ, 117, 1490

Pezzuto, S., Elia, D., Schisano, E., et al. 2012, A\&A, 547, A54

Phillips, R. R., Gibb, A. G., \& Little, L. T. 2001, MNRAS, 326, 927

Pilbratt, G. L., Riedinger, J. R., Passvogel, T., et al. 2010, A\&A, 518, L1

Pineda, J. E., Arce, H. G., Schnee, S., et al. 2011, ApJ, 743, 201

Poglitsch, A., Waelkens, C., Geis, N., et al. 2010, A\&A, 518, L2

Porter, T. A., \& Strong, A. W. 2005, in Proc. 29th ICRC (Pune), 4, 77

Press, W. H., Teukolsky, S. A., Vetterling, W. T., \& Flanney, B. P. 1992, Numerical Recipes in FORTRAN (Cambridge: Cambridge Univ. Press)

Price, D. J., Tricco, T. S., \& Bate, M. R. 2012, MNRAS, 423, L45

Ragan, S., Henning, T., Krause, O., et al. 2012, A\&A, 547, A49

Reggiani, M., Robberto, M., Da Rio, N., et al. 2011, A\&A, 534, A83

Reipurth, B., Megeath, S. T., Bally, J., \& Walawender, J. 2008, in Handbook of Star Forming Regions, Vol. I: The Northern Sky, ed. B. Reipurth (ASP Monograph Publications, Vol. 4; San Francisco, CA: ASP), 782
Reipurth, B., Rodríguez, L. F., \& Chini, R. 1999, AJ, 118, 983

Robitaille, T. P. 2011, A\&A, 536, A79

Robitaille, T. P., Meade, M. R., Babler, B. L., et al. 2008, AJ, 136, 2413

Roussel, H. 2012, PASP, submitted (arXiv:1205.2576)

Sadavoy, S. I., Di Francesco, J., Bontemps, S., et al. 2010, ApJ, 710, 1247

Sandstrom, K. M., Peek, J. E. G., Bower, G. C., Bolatto, A. D., \& Plambeck, R. L. 2007, ApJ, 667, 1161

Savva, D., Little, L. T., Phillips, R. R., \& Gibb, A. G. 2003, MNRAS, 343, 259

Siringo, G., Kreysa, E., De Breuck, C., et al. 2010, Msngr, 139, 20

Siringo, G., Kreysa, E., Kovács, A., et al. 2009, A\&A, 497, 945

Sodroski, T. J., Odegard, N., Arendt, R. G., et al. 1997, ApJ, 480, 173

Stanke, T., McCaughrean, M. J., \& Zinnecker, H. 2002, A\&A, 392, 239

Stanke, T., Stutz, A. M., Tobin, J. J., et al. 2010, A\&A, 518, L94

Stern, D., Eisenhardt, P., Gorjian, V., et al. 2005, ApJ, 631, 163

Strom, K. M., Strom, S. E., \& Vrba, F. J. 1976, AJ, 81, 308

Strom, K. M., Strom, S. E., Wolff, S. C., Morgan, J., \& Wenz, M. 1986, ApJS, 62,39

Stutz, A., Launhardt, R., Linz, H., et al. 2010, A\&A, 518, L87

Stutz, A. M., Rubin, M., Werner, M. W., et al. 2008, ApJ, 687, 389

Terebey, S., Shu, F. H., \& Cassen, P. 1984, ApJ, 286, 529

Tobin, J. J., Hartmann, L., Chiang, H.-F., et al. 2012, Natur, 492, 83

Tobin, J. J., Looney, L. W., Mundy, L. G., Kwon, W., \& Hamidouche, M. 2007, ApJ, 659, 1404

Tsujimoto, M., Koyama, K., Kobayashi, N., et al. 2003, AJ, 125, 1537

Ulrich, R. K. 1976, ApJ, 210, 377

Whitney, B. A., Wood, K., Bjorkman, J. E., \& Cohen, M. 2003a, ApJ, 598, 1079

Whitney, B. A., Wood, K., Bjorkman, J. E., \& Wolff, M. J. 2003b, ApJ, 591,1049

Williams, J. P., \& Cieza, L. A. 2011, ARA\&A, 49, 67

Winston, E., Megeath, S. T., Wolk, S. J., et al. 2007, ApJ, 669, 493

Wu, Y., Wei, Y., Zhao, M., et al. 2004, A\&A, 426, 503

Young, C. H., \& Evans, N. J., II. 2005, ApJ, 627, 293

Zavagno, A., Molinari, S., Tommasi, E., Saraceno, P., \& Griffin, M. 1997, A\&A, 325,685 